Desenvolvimento e otimização de um código paralelizado para simulação de escoamentos incompressíveis

Josuel Kruppa Rogenski 



\title{
Desenvolvimento e otimização de um código paralelizado para simulação de escoamentos incompressíveis
}

\author{
Josuel Kruppa Rogenski
}

Orientador: Prof. Dr. Leandro Franco de Souza

Dissertação apresentada ao Instituto de Ciências Matemáticas e de Computação - ICMC-USP, como parte dos requisitos para obtenção do título de Mestre em Ciências - Ciências de Computação e Matemática Computacional. VERSÃO REVISADA.

USP - São Carlos

Maio/2011 
Ficha catalográfica elaborada pela Biblioteca Prof. Achille Bassi e Seção Técnica de Informática, ICMC/USP, com os dados fornecidos pelo(a) autor(a)

\begin{tabular}{|c|c|}
\hline \multirow[t]{3}{*}{ R721d } & $\begin{array}{l}\text { Rogenski, Josuel Kruppa } \\
\text { Desenvolvimento e otimização de um código } \\
\text { paralelizado para simulação de escoamentos } \\
\text { incompressíveis / Josuel Kruppa Rogenski; orientador } \\
\text { Leandro Franco de Souza -- São Carlos, } 2011 . \\
\quad 87 \mathrm{p} \text {. }\end{array}$ \\
\hline & $\begin{array}{l}\text { Dissertação (Mestrado - Programa de Pós-Graduação en } \\
\text { Ciências de Computação e Matemática Computacional) -- } \\
\text { Instituto de Ciências Matemáticas e de Computação, } \\
\text { Universidade de São Paulo, } 2011 .\end{array}$ \\
\hline & $\begin{array}{l}\text { 1. computação paralela. } 2 \text {. equações de Navier- } \\
\text { Stokes. 3. diferenças finitas compactas de alta } \\
\text { ordem. 4. métodos multigrid. I. Franco de Souza, } \\
\text { Leandro, orient. II. Título. }\end{array}$ \\
\hline
\end{tabular}


a meus pais. 



\section{Agradecimentos}

\section{A Deus.}

A minha família.

Ao professor Dr. Leandro Franco de Souza, pela oportunidade, orientação, disposição e paciência. À Fundação de Amparo à Pesquisa do Estado de São Paulo, pelo suporte financeiro.

Ao Instituto de Ciências Matemáticas e de Computação e ao Conselho Nacional de Desenvolvimento Científico e Tecnológico.

Ao corpo docente do LCAD, pelos ensinamentos.

À Larissa Alves Petri e a Leonardo Martinussi, pela pronta ajuda.

Ao pessoal do LCAD, em especial à Laís Corrêa, Patrícia Satori, Rafael Alves Figueiredo, Miguel Caro, Giseli Aparecida Braz de Lima e Ítalo Tasso, pelos bons momentos compartilhados.

Aos amigos Cleber de Medeira e Luis Felipe Santos Mendes pelas úteis discussões inúteis.

Aos professores Elisangela dos Santos Meza, Moisés Meza Pariona e Luciane Grossi Bombacini, pelo incentivo em tempos de graduação.

E a todos que contribuíram, direta ou indiretamente, no desenvolvimento do presente trabalho. 


\section{Resumo}

O presente trabalho de pesquisa tem por objetivo estudar a paralelização de algoritmos voltados à solução de equações diferenciais parciais. Esses algoritmos são utilizados para gerar a solução numérica das equações de Navier-Stokes em um escoamento bidimensional incompressível de um fluido newtoniano. As derivadas espaciais são calculadas através de um método de diferenças finitas compactas com a utilização de aproximações de altas ordens de precisão. Uma vez que o cálculo de derivadas espaciais com alta ordem de precisão da forma compacta adotado no presente estudo requer a solução de sistemas lineares tridiagonais, é importante realizar estudos voltados a resolução desses sistemas, para se obter uma boa performance. Ressalta-se ainda que a solução de sistemas lineares também faz-se presente na solução numérica da equação de Poisson. Os resultados obtidos decorrentes da solução das equações diferenciais parciais são comparados com os resultados onde se conhece a solução analítica, de forma a verificar a precisão dos métodos implementados. Os resultados do código voltado à resolução das equações de Navier-Stokes paralelizado para simulação de escoamentos incompressíveis são comparados com resultados da teoria de estabilidade linear, para validação do código final. Verifica-se a performance e o speedup do código em questão, comparando-se o tempo total gasto em função do número de elementos de processamento utilizados.

Palavras-chave: computação paralela, equações de Navier-Stokes, diferenças finitas compactas de alta ordem, métodos multigrid 


\section{Abstract}

The objective of the present work is to study the parallelization of partial differential equations. The aim is to achieve an effective parallelization to generate numerical solution of Navier-Stokes equations in a two-dimensional incompressible and isothermal flow of a Newtonian fluid. The spatial derivatives are calculated using compact finite differences approximations of higher order accuracy. Since the calculation of spatial derivatives with high order adopted in the present work requires the solution of tridiagonal systems, it is important to conduct studies to solve these systems and achieve good performance. In addiction, linear systems solution is also present in the numerical solution of a Poisson equation. The results generated by the solution of partial differential equations are compared to analytical solution, in order to verify the accuracy of the implemented methods. The numerical parallel solution of a Navier-Stokes equations is compared with linear stability theory to validate the final code. The performance and the speedup of the code in question is also checked, comparing the execution time in function of the number of processing elements.

Keywords: parallel computing, Navier-Stokes equations, high-order compact finite difference, multigrid methods 


\section{Sumário}

1 Introdução 1

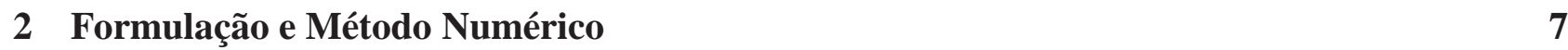

2.1 As equações de Navier-Stokes . . . . . . . . . . . . . . . . . . . . 7

2.2 Transição laminar-turbulento . . . . . . . . . . . . . . . . . . . . . . 9

2.2.1 Teoria de Estabilidade Linear _ . . . . . . . . . . . . . . . . 9

2.2.2 Análise espacial e temporal de instabilidades . . . . . . . . . . . . . . 13

2.2.3 Solução numérica da equação de Orr-Sommerfeld . . . . . . . . . . . . . . 13

2.3 Método numérico . . . . . . . . . . . . . . . . . . . . . . . 15

2.3.1 Introdução de perturbações . . . . . . . . . . . . . . . . . . 15

2.3.2 Esquemas multigrid . . . . . . . . . . . . . . . . . 16

2.3.3 Derivadas Temporais . . . . . . . . . . . . . . . . . 23

2.3.4 Aproximação numérica de derivadas espaciais . . . . . . . . . . . . . . . 24

2.3.5 Amortecimento de reflexões na entrada do escoamento . . . . . . . . . . . . 25

2.3.6 Relaminarização do escoamento . . . . . . . . . . . . . . . . . 25

2.3.7 Cálculo da vorticidade na parede . . . . . . . . . . . . . . . 26

2.3 .8 Filtragem Espacial . . . . . . . . . . . . . . . . . . . . . 27

2.3 .9 O método numérico . . . . . . . . . . . . . . . . . . . . 28

3 Técnicas de paralelização $r$

3.1 Paralelização de métodos multigrid . . . . . . . . . . . . . . . . . . . . 29

3.2 Diferenças compactas de alta ordem paralelizadas . . . . . . . . . . . . . . . 31

3.2.1 Algoritmo sequencial - Algoritmo clássico de Thomas . . . . . . . . . . . . 31

3.2.2 Estratégia de paralelização I . . . . . . . . . . . . . . . . . . . 32

3.2 .3 Estratégia de paralelização II . . . . . . . . . . . . . . . . . . . . . . 34

3.3 A paralelização na filtragem espacial . . . . . . . . . . . . . . . . . . 35

3.3.1 Filtragem espacial - Estratégia de paralelização I . . . . . . . . . . . . 35

3.3.2 Filtragem espacial - Estratégia de paralelização II . . . . . . . . . . . 36 
4 Resultados $\quad 37$

4.1 Paralelização de métodos multigrid . . . . . . . . . . . . . . . . . . 37

$4.1 .1 \quad$ Esquemas multigrid versus singlegrid . . . . . . . . . . . . . . . . 38

4.1.2 Influência da alocação da estrutura de dados . . . . . . . . . . . . . . . . 39

4.1.3 Comparação de métodos multigrid CS e FAS . . . . . . . . . . . . . . . . 41

4.2 Diferenças compactas de alta ordem paralelizadas . . . . . . . . . . . . . . . 45

4.3 A paralelização na filtragem espacial . . . . . . . . . . . . . . . . . . 46

4.4 Escoamento de Poiseuille . . . . . . . . . . . . . . . . . . . . . . . 48

4.4.1 Escoamento de Poiseuille e a Teoria de Estabilidade Linear . . . . . . . . . . 48

4.4.2 Escoamento de Poiseuille - Comparação entre os métodos multigrid CS e FAS 51

4.4.3 Escoamento de Poiseuille - Comparação entre estratégias de paralelização para diferenças compactas . . . . . . . . . . . . . . 55

5 Conclusões e trabalhos futuros $\quad 57$

5.1 Síntese do trabalho realizado . . . . . . . . . . . . . . . . 57

5.2 Considerações . . . . . . . . . . . . . . . . . . . . . 57

5.3 Trabalhos futuros $\ldots \ldots \ldots \ldots \ldots \ldots \ldots$

$\begin{array}{ll}\text { Referências } & 63\end{array}$

$\begin{array}{ll}\text { A Dados quantitativos } & 65\end{array}$ 


\section{Lista de Figuras}

2.1 2.1(a) Solução analítica da função $v$ e 2.1(b) Chute inicial utilizado.

2.2 Comportamento do módulo do erro após: (a) nenhuma iteração; (b) 1 iteração; (c) 25 iterações; (d) 1000 iterações. . . . . . . . . . . . . . . . . . . . . . 17

2.3 Esquema multigrid - Ciclo V . . . . . . . . . . . . . . . . . . . . . 18

2.4 Esquema de correção CS - Ciclo V . . . . . . . . . . . . . . . . . . . 18

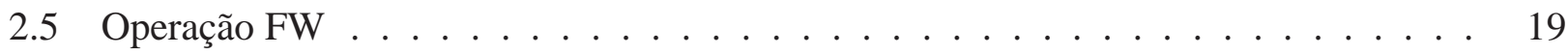

2.6 Interpolação bilinear . . . . . . . . . . . . . . . . . . . . . . 20

2.7 Método multigrid FAS - Ciclo V . . . . . . . . . . . . . . . . . . . . . 21

2.8 Operação SI . . . . . . . . . . . . . . . . . . . . . . . . . . 21

3.1 Intersecção entre domínios . . . . . . . . . . . . . . . . . . . 30

3.2 Pontos de comunicação: 3.2(a) esquema CS; 3.2(b) esquema FAS . . . . . . . . . . . 31

3.3 Comportamento do erro: 3.3(a) caso sequencial; 3.3(b) decomposição de domínio. . 34

3.4 Estratégia de paralelização II - Comunicação entre elementos de processamentos. . . 35

4.1 Razão de melhoramento entre os métodos SOR sequencial e o método multigrid CS

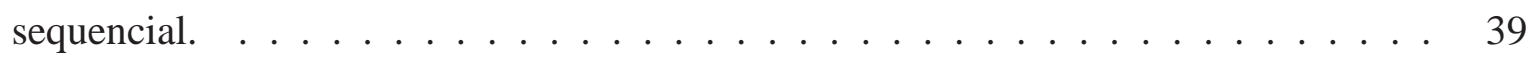

4.2 Influência da alocação de estrutura de dados utilizando o método multigrid FAS. . . . 40

4.3 Tempo de execução dos métodos multigrid CS e FAS sequenciais. . . . . . . . . . . 41

4.4 Speedup dos métodos multigrid paralelizados para o caso I. . . . . . . . . . . . . . 42

4.5 Eficiência dos métodos multigrid paralelizados para o caso I. . . . . . . . . . . . . 43

4.6 Speedup dos métodos multigrid paralelizados para o caso II. . . . . . . . . . . . . . 44

4.7 Eficiência dos métodos multigrid paralelizados para o caso II. . . . . . . . . . . . . . 44

4.8 Speedup e Eficiência referentes ao cálculo da $1^{a}$ derivada. . . . . . . . . . . . . . . 46

4.9 Speedup e Eficiência referentes ao cálculo da $2^{a}$ derivada. . . . . . . . . . . . . . . . 46

4.10 Speedup e Eficiência referentes a aplicação do filtro. . . . . . . . . . . . . . . . . 47

4.11 Diferença existente entre a aplicação do filtro sequencial e do filtro paralelizado utilizando a estratégia de paralelização II. . . . . . . . . . . . . . . . . . . . . . . 48 
4.12 Escoamento de Poiseuille - domínio considerado. . . . . . . . . . . . . . . . . . 49

4.13 Casos considerados frente à curva neutra. . . . . . . . . . . . . . . . . 50

4.14 Escoamento de Poiseuille - Caso A - dados qualitativos. . . . . . . . . . . . . . . . 51

4.15 Escoamento de Poiseuille - Caso B - dados qualitativos. . . . . . . . . . . . . . 52

4.16 Escoamento de Poiseuille - Caso C - dados qualitativos. . . . . . . . . . . . . . . 53

4.17 Escoamento de Poiseuille - Análise de Fourier - Comportamento referente ao primeiro modo. . . . . . . . . . . . . . . . . . . . . 54

4.18 Escoamento de Poiseuille - comparativo entre métodos multigrid sequenciais. . . . . 54

4.19 Escoamento de Poiseuille - comparativo entre métodos multigrid utilizando a estratégia I de paralelização de aproximação de diferenças compactas. . . . . . . . . . . . . . 55

4.20 Escoamento de Poiseuille - comparativo entre métodos multigrid utilizando a estratégia II de paralelização de aproximação de diferenças compactas. . . . . . . . . . . . . 55

4.21 Escoamento de Poiseuille - comparativo entre estratégias de paralelização de aproximação de diferenças compactas utilizando o método multigrid FAS. . . . . . . . . . . 56 


\section{Lista de Tabelas}

2.1 Classificação de instabilidades. . . . . . . . . . . . . . . . . . . . . 13

3.1 Estratégia de paralelização I - Aproximação de derivadas por diferenças compactas de alta ordem utilizando o conceito de pipeline. . . . . . . . . . . . . . . . . 33

4.1 Eixo nominal - malhas utilizadas - molécula computacional de 5 pontos. . . . . . . 38

4.2 Eixo nominal - malhas utilizadas - sistemas tri e pentadiagonais. . . . . . . . . . . 45

4.3 Escoamento de Poiseuille - Casos investigados. . . . . . . . . . . . . . . . . . . . 49

4.4 Escoamento de Poiseuille - Comparativo realizado utilizando a teoria de estabilidade linear. . . . . . . . . . . . . . . . . . . . . 50 50

4.5 Escoamento de Poiseuille - Eixo nominal - malhas utilizadas. . . . . . . . . . . . 52

A.1 Método multigrid CS - Caso I - 1p. . . . . . . . . . . . . . . . . . . . . . 66

A.2 Método multigrid CS - Caso I - 2p. . . . . . . . . . . . . . . . . . . . . . 67

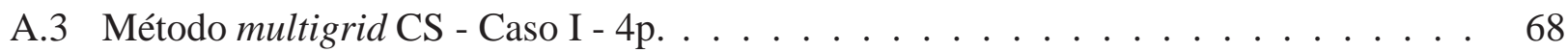

A.4 Método multigrid CS - Caso I - 8p. . . . . . . . . . . . . . . . . . . . . . 69

A.5 Método multigrid FAS - Caso I - 1 p . . . . . . . . . . . . . . . . . . . 70

A.6 Método multigrid FAS - Caso I - 2p. . . . . . . . . . . . . . . . . . . . 71

A.7 Método multigrid FAS - Caso I - 4p . . . . . . . . . . . . . . . . . 72

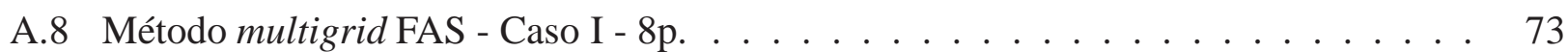

A.9 Método multigrid CS - Caso II - 1p. . . . . . . . . . . . . . . . . . . . . . . . . 74

A.10 Método multigrid CS - Caso II - 2p. . . . . . . . . . . . . . . . . . 75

A.11 Método multigrid CS - Caso II - 4p. . . . . . . . . . . . . . . . . 76

A.12 Método multigrid CS - Caso II - 8p. . . . . . . . . . . . . . . . . . . . . . 77

A.13 Método multigrid FAS - Caso II - 1p. . . . . . . . . . . . . . . . . . . . . . 78

A.14 Método multigrid FAS - Caso II - 2p. . . . . . . . . . . . . . . . . . . . . 79

A.15 Método multigrid FAS - Caso II - 4p . . . . . . . . . . . . . . . . . . 80

A.16 Método multigrid FAS - Caso II - 8p. . . . . . . . . . . . . . . . . . . . . 81 
A.17 Escoamento de Poiseuille - Caso B - 665 pontos - Estratégia de paralelização I. . . 82

A.18 Escoamento de Poiseuille - Caso B - 1049 pontos - Estratégia de paralelização I. . . . 83

A.19 Escoamento de Poiseuille - Caso B - 1433 pontos - Estratégia de paralelização I. 84

A.20 Escoamento de Poiseuille - Caso B - 665 pontos - Estratégia de paralelização II. . . . 85

A.21 Escoamento de Poiseuille - Caso B - 1049 pontos - Estratégia de paralelização II. . . 86

A.22 Escoamento de Poiseuille - Caso B - 1433 pontos - Estratégia de paralelização II. . 87 


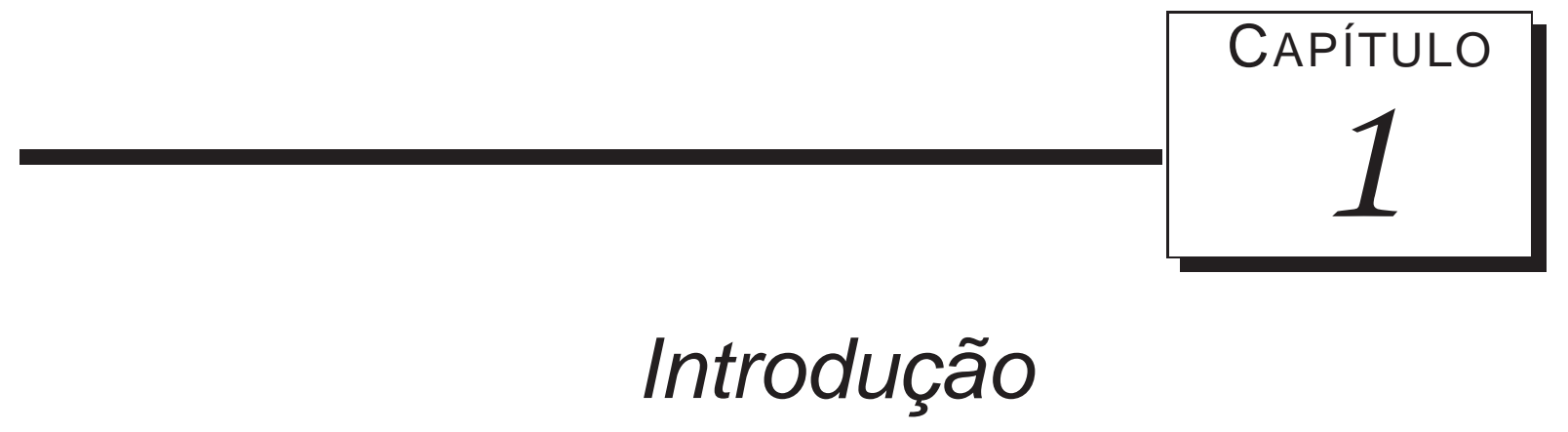

O movimento de oceanos, rios e lagos, bem como o sistema de circulação sanguínea e o movimento dos diversos meios de transportes fazem parte de um grande conjunto de fenômenos presentes no cotidiano que podem ser estudados por meio da mecânica dos fluidos.

Um marco significativo na história da mecânica dos fluidos moderna deu-se por Ludwig Prandtl, em 1904. Prandtl alcançou um alto grau de correlação entre campos analíticos e experimentais da dinâmica dos fluidos, ao desenvolver a teoria da camada limite. Por meio da teoria da camada limite, importantes avanços na área de transição à turbulência puderam ser alcançados.

O fenômeno de transição de um escoamento de um regime laminar a um regime turbulento foi inicialmente estudado, segundo Schlichting (1979), por Osborne Reynolds, ao final do século XIX. Reynolds observou o comportamento de escoamentos confinados por meio de um traçador no centro de um determinado conduto e verificou que, de acordo com um determinado parâmetro adimensional, o escoamento poderia ser laminar ou turbulento.

Fundamentado pelos estudos de Reynolds, Lord Rayleigh, em 1913, verificou que a instabilidade de um escoamento está associada à existência de um ponto de inflexão no perfil de velocidade desse escoamento. Além disso, os pesquisadores Orr e Sommerfeld desenvolveram respectivamente, nos anos 1907 e 1908, estudos teóricos independentes voltados a propagação de perturbações em uma camada limite. Esses estudos deram origem à equação de Orr-Sommerfeld. Tollmien e Schlichting, em 1933, demonstraram que essa equação possui perturbações instáveis para escoamentos, sem que hajam pontos de inflexão.

Tendo como referência o cenário científico atual, preocupações voltadas à busca pela redução de tempo de execução e geração de códigos capazes de alcançar altas ordens têm-se tornado frequentes. Além disso, conceitos associados à paralelização tornam-se cada vez mais comuns devido a ampla difusão de processadores multicore no cenário tecnológico. 
De maneira específica, torna-se interessante ressaltar que grande parte das formulações numéricas voltadas à simulação de escoamentos incompressíveis dependem da solução de uma equação de Poisson. A resolução numérica dessa equação diferencial parcial exige um alto custo computacional, já que faz-se necessário a resolução de um sistema linear. Neste sentido, a paralelização de métodos iterativos e a utilização de esquemas multigrid de alta ordem são estratégias que podem oferecer ganhos significativos no desempenho de códigos computacionais e minimizar os impactos decorrentes da complexidade e do alto custo de processamento.

Altas ordens podem ser alcançadas por meio da utilização de diferenças compactas. Mesmo exigindo um aumento no custo computacional, o uso dessa estratégia é justificada, segundo Lele (1992); Hirsh (1975); Wray e Hussaini (1994); Kloker (1998); Mahesh (1998) e Souza et al. (2005), pela baixa dispersão e dissipação numéricas ${ }^{1}$, redução do número de pontos a serem utilizados, diminuição do erro e alta resolução.

O método multigrid foi desenvolvido por Fedorenko(1964) com o propósito de resolver a equação de Poisson em um domínio retangular. No entanto, foram os estudos de Brandt (1977) que possibilitaram uma maior divulgação do método no cenário científico. A principal vantagem desse método é a rápida convergência. $\mathrm{O}$ método multigrid é mais eficiente computacionalmente por resolver equações diferenciais parciais elípticas discretizadas em $N$ pontos com uma complexidade da ordem do número de pontos, de acordo com Zhang (1996, 1997); Spitaleri (2000) e Gupta et al. (1997). A eficiência desses métodos, segundo de Velde (1994), refere-se a combinar métodos iterativos em malhas com diferentes espaçamentos. Isso se deve, segundo o autor, ao fato de que métodos iterativos reduzem efetivamente - com um pequeno número de iterações - erros associados apenas a altas frequências. Todavia, segundo Briggs et al. (2000), métodos multigrid podem exigir implementações específicas para cada tipo de problema a ser estudado.

De forma geral, há numerosas contribuições científicas, na área da dinâmica de fluidos computacional relacionadas à utilização de aproximações de altas ordens, aplicação de métodos multigrid e paralelização de processos, no sentido de se obter ganhos de desempenho frente a estratégias usuais. Dentre elas, citam-se:

Abdullah e Ali (1996) introduzem possíveis estratégias de paralelização dos métodos - Explict Group e Explicit Decoupled Group - para a resolução de equações diferenciais parciais elípticas. As estratégias consistem basicamente em dividir o domínio em faixas com largura de duas linhas ou em blocos contendo quatro incógnitas. Todas as estratégias de paralelização apresentaram ganhos frente a versões sequenciais.

John e Tobiska (2000) apresentam resultados voltados à utilização de métodos multigrid acoplados para resolução paralelizada das equações de Navier-Stokes incompressíveis. Destacam-se duas classes de suavizadores para método multigrid. A primeira classe considerada é conhecida pela utilização de métodos do tipo Gauss-Seidel por blocos, em cada passo da aplicação do suavizador. Em

\footnotetext{
${ }^{1} \mathrm{O}$ conceito de dispersão está associado à presença de oscilações na solução numérica. Já o conceito de dissipação volta-se para a suavização da solução.
} 
contrapartida, a segunda estratégia implica na resolução de um grande sistema por meio de métodos do tipo gradiente e visa a utilização de métodos similares ao método SIMPLE como suavizadores. A paralelização dá-se por meio de adaptações realizadas em um pacote de rotinas ugp 1.0 desenvolvidas por Bastian apud John e Tobiska (2000).

Brown e Ingber (2002) discutem a implementação de um código computacional paralelizado, utilizando um método de elementos finitos, voltado à simulação de escoamentos víscidos incompressíveis. Adota-se uma formulação vorticidade dividida em dois passos cinemáticos e um cinético. A paralelização ocorre por meio da utilização do pacote ScaLAPACK. O código paralelizado, segundo os autores, apresenta vantagens de escalabilidade ${ }^{2}$.

Zhang (2002) apresenta estudos voltados à resolução da equação de Poisson 2D, os quais retratam a influência do método multigrid usando métodos do tipo Gauss-Seidel e estratégias de semi engrossamento. Além disso, há utilização de esquemas compactos de quarta ordem em malhas não uniformes. Os métodos apresentados pelo autor comportam-se bem frente à resolução de esquemas compactos de quarta ordem voltados à resolução da equação de Poisson bidimensional. Ge (2010) estendeu os estudos de Zhang (2002) para o caso da equação de Poisson tridimensional.

Sterk e Trobec (2003) apresentam um estudo, implementação e comparação de dois métodos paralelizados - Successive Over-Relaxation(SOR) e um método multigrid. Para tanto, considera-se um domínio tridimensional arbitrário com condições de contorno do tipo Neumann. As derivadas são aproximadas por meio do método das diferenças finitas. O sistema linear decorrente dessa aproximação é resolvido utilizando os métodos iterativos anteriormente citados e constata-se ganhos no paralelismo, no que se refere a rapidez em domínios com um número significativo de pontos.

de Angeli et al. (2003) introduzem discussões a respeito da implementação de algoritmos paralelizados baseados no método de diferenças finitas, no sentido de simular escoamentos incompressíveis. Utilizam-se um esquema explícito e implícito, para o tratamento dado à pressão e à velocidade, respectivamente. A paralelização é baseada em decomposição de domínio e contempla a inicialização, a resolução de sistemas lineares utilizando o método SOR e a comunicação entre domínios. Estudos são realizados no sentido de verificar a influência do número de processos e estratégias de comunicação em relação à performance dos códigos paralelizados. Comparações associadas à influência de estratégias de comunicação utilizadas mostram que a utilização de um pequeno número de processos não implica mudanças significativas entre a performance dos códigos obtidos. No entanto, caso seja utilizado um número significativo de processos, a utilização de funções da biblioteca Message Passing Interface (MPI) aplicadas ao conceito de árvore binária apresenta os melhores resultados. Investiga-se também a influência do número de pontos de comunicação frente ao desempenho do código. De forma geral, os autores afirmam que valores ótimos dependem da classe de problemas a serem estudados.

Ali e Fu (2008) realizam um comparativo associado à obtenção da solução da equação de Pois-

\footnotetext{
${ }^{2} \mathrm{O}$ conceito de escalabilidade está associado ao aumento/diminuição proporcional dos índices de speedup em relação à um aumento/diminuição do número de processos.
} 
son bidimensional, com condição de contorno de Dirichlet. Os métodos Explicit Group e Explicit Decoupled Group são confrontados com dois métodos, criados pelos autores e denominados Modified Explicit Group e Parallel Modified Explicit Group. A adaptação proposta pelos autores está associada a empregar o método Explicit Decoupled Group considerando um espaçamento de $2 h$. De posse da solução numérica nestes pontos, obtém-se diretamente a solução nos demais pontos. Como resultados preliminares, os autores afirmam que o método paralelizado obteve os melhores índices de velocidade e acurácia. O método paralelizado mostrou-se também ser escalável com respeito ao número de processos.

Simens et al. (2009) propõem um novo código de alta resolução voltado à simulação de uma camada limite incompressível sob uma placa plana, onde o avanço temporal dá-se por meio de um método de Runge-Kutta de três passos e os termos convectivos e viscosos são aproximados por meio de diferenças compactas de estêncil igual a três. A paralelização dá-se por meio da biblioteca MPI. Segundo o autor, o código implementado é facilmente transportável a outras plataformas (similares) e demanda, em comunicação, $35 \%$ do tempo de execução.

Wang e Zhang (2009) descrevem o desenvolvimento de um método multigrid geométrico, similar ao método Full Multigrid, como forma de obter a solução da equação de Poisson 2D com sexta ordem. Essencialmente, segundo os autores, a equação é discretizada utilizando uma aproximação compacta de quarta ordem. A sexta ordem é alcançada por meio da aplicação da técnica de extrapolação de Richardson associada ao operador de interpolação do método multigrid. A estratégia mostra-se superior aos demais métodos de sexta ordem comparados, mantendo ainda baixo custo de processamento.

Henniger et al. (2010) apresentam um código paralelizado de alta ordem voltado à simulação de grandes escoamentos incompressíveis tridimensionais. Os autores utilizam diferenças finitas no que se refere à discretização espacial e um esquema de integração semi-implícito para avanço temporal. A paralelização dá-se pela decomposição de domínio em três dimensões. O autor demonstra que o método proposto é escalável utilizando um número de processos da ordem de $10^{4}$ e malhas contendo número de pontos na ordem de $10^{11}$.

Buckeridge e Scheichl (2010) realizam estudos voltados à resolução da equação de Poisson em coordenadas polares esféricas, por meio de um método multigrid geométrico paralelizado. A discretização é realizada por meio do método dos volumes finitos. A decomposição de domínio dá-se nas direções longitudinal e latitudinal. Emprega-se a biblioteca MPI para fins de paralelização. Em relação as medidas de speedup, os índices apresentados são quase ótimos utilizando até 8 processos. Os autores realizam diversos comparativos voltados a performance de códigos paralelizados. Com isso, demonstram ganhos em se aplicar métodos multigrid em relação a aplicação de métodos embasados, por exemplo, em subespaços de Krylov.

Neste contexto, o presente trabalho de pesquisa tem como objetivo o estudo e implementação de um código paralelizado de alta ordem para a solução das equações de Navier-Stokes, no sentido de investigar como ocorre o fenômeno de transição à turbulência, para um escoamento incompressível e isotérmico bidimensional de um fluido newtoniano. Especificamente, adota-se a formulação 
vorticidade-velocidade. Justifica-se o uso dessa formulação pela facilidade decorrente da eliminação do tratamento dado a pressão e pela utilização de malhas colocalizadas. O sistema linear associado a equação de Poisson é resolvido por meio de métodos multigrid. As derivadas espaciais são aproximadas por meio de diferenças compactas de alta ordem. O avanço temporal dá-se por meio de um método do tipo Runge-Kutta de quarta ordem. Além disso, a paralelização ocorre por meio de decomposição de domínio na direção $x$. Para tanto, utiliza-se a biblioteca MPI.

A presente dissertação está estruturada da seguinte forma: no capítulo 2 a discretização das equações de Navier-Stokes bidimensionais é realizada por meio da formulação vorticidade-velocidade. $\mathrm{Na}$ sequência, são apresentados conceitos referentes à teoria de estabilidade linear. Tópicos relevantes ao método numérico também são apresentados, como a descrição de métodos multigrid, aproximação por diferenças finitas compactas de alta ordem, tratamento dado a vorticidade, inserção de perturbações e aplicação de um filtro computacional. O capítulo 3 trata de conceitos e estratégias voltadas à paralelização do método numérico. Na sequência, no capítulo 4, são descritos os principais resultados obtidos. Tópicos referentes a vantagens na utilização de métodos multigrid, forma de alocação de estrutura de dados, e comparativos entre diferentes métodos paralelizados são realizados. Finalmente, o capítulo 5 volta-se para uma síntese geral do trabalho realizado. Tabelas contendo os principais dados gerados, são apresentadas no Apêndice. 


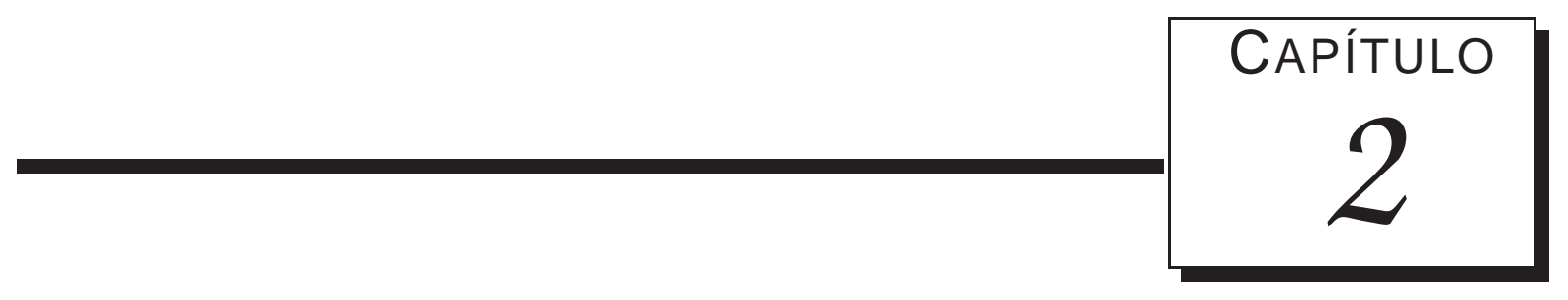

\section{Formulação e Método Numérico}

O presente capítulo apresenta a formulação numérica das equações de Navier-Stokes bidimensionais. Na sequência são abordados tópicos associados à transição laminar-turbulento, principalmente voltados à teoria de estabilidade linear. São também discutidos tópicos relevantes ao método numérico, tais como: a caracterização de métodos multigrid, aproximação de derivadas temporais e espaciais, tratamento dado à vorticidade, inserção de perturbações e aplicação de um filtro computacional.

\subsection{As equações de Navier-Stokes}

Escoamentos incompressíveis e isotérmicos, de fluidos newtonianos, podem ser modelados por meio das equações de Navier-Stokes. O sistema de equações gerado, juntamente com a equação da continuidade, é expresso na forma vetorial, por:

$$
\begin{aligned}
\nabla \cdot \vec{u}^{*} & =0, \\
\frac{\partial \vec{u}^{*}}{\partial t^{*}}+\left(\vec{u}^{*} \cdot \nabla\right) \vec{u}^{*} & =-\frac{1}{\rho^{*}} \nabla p^{*}+\nu^{*} \nabla^{2} \vec{u}^{*}
\end{aligned}
$$

em que $\vec{u}^{*}$ é o vetor velocidade, $p^{*}$ é a pressão, $t^{*}$ é o tempo, $\rho^{*}$ é a densidade e $\nu^{*}$ é a viscosidade cinemática. Especificamente, considerando um escoamento bidimensional, o sistema de equações na 
forma adimensional é dado como segue:

$$
\begin{aligned}
\frac{\partial u}{\partial x}+\frac{\partial v}{\partial y} & =0 \\
\frac{\partial u}{\partial t}+u \frac{\partial u}{\partial x}+v \frac{\partial u}{\partial y} & =-\frac{\partial p}{\partial x}+\frac{1}{R e}\left(\frac{\partial^{2} u}{\partial x^{2}}+\frac{\partial^{2} u}{\partial y^{2}}\right), \\
\frac{\partial v}{\partial t}+u \frac{\partial v}{\partial x}+v \frac{\partial v}{\partial y} & =-\frac{\partial p}{\partial y}+\frac{1}{R e}\left(\frac{\partial^{2} v}{\partial x^{2}}+\frac{\partial^{2} v}{\partial y^{2}}\right) .
\end{aligned}
$$

Os parâmetros adimensionais utilizados são:

$$
x=\frac{x^{*}}{L^{*}}, \quad y=\frac{y^{*}}{L^{*}}, \quad u=\frac{u^{*}}{U_{\infty}^{*}}, \quad v=\frac{v^{*}}{U_{\infty}^{*}}, \quad p=\frac{p^{*}}{\rho^{*} U_{\infty}^{*}}, \quad t=\frac{t^{*} U_{\infty}^{*}}{L^{*}},
$$

juntamente com,

$$
R e=\frac{U_{\infty}^{*} L^{*}}{\nu^{*}}
$$

Ressalta-se que as variáveis dimensionais são indicadas por $*$. O parâmetro $L^{*}$ é tomado como um comprimento de referência, $U_{\infty}^{*}$ é a velocidade de referência do escoamento sem perturbação e $R e$ é o número de Reynolds. Além disso, $x^{*}$ e $y^{*}$ representam as coordenadas nas direções longitudinal e normal, respectivamente.

Tendo como objetivo eliminar o tratamento dado à pressão nas equações de Navier-Stokes, opta-se pela utilização da formulação vorticidade-velocidade. O uso dessa formulação ainda permite a utilização de malhas colocalizadas, facilitando a implementação de métodos de alta ordem. Para tanto, define-se a vorticidade $\omega_{z}$ bidimensional, como:

$$
\omega_{z}=\frac{\partial u}{\partial y}-\frac{\partial v}{\partial x}
$$

Por meio da definição de vorticidade, e considerando a equação da continuidade, deriva-se uma equação de Poisson para o componente $v$ da velocidade:

$$
\frac{\partial^{2} v}{\partial x^{2}}+\frac{\partial^{2} v}{\partial y^{2}}=-\frac{\partial \omega_{z}}{\partial x} .
$$

Além disso, convenientes transformações envolvendo as equações de Navier-Stokes resultam na equação de transporte de vorticidade:

$$
\frac{\partial \omega_{z}}{\partial t}=-\frac{\partial\left(u \omega_{z}\right)}{\partial x}-\frac{\partial\left(v \omega_{z}\right)}{\partial y}+\frac{1}{R e}\left(\frac{\partial^{2} \omega_{z}}{\partial x^{2}}+\frac{\partial^{2} \omega_{z}}{\partial y^{2}}\right) .
$$

Sendo assim, tendo como referência a formulação adotada, o seguinte sistema de equações deve 
ser considerado:

$$
\begin{aligned}
\frac{\partial \omega_{z}}{\partial t} & =-\frac{\partial\left(u \omega_{z}\right)}{\partial x}-\frac{\partial\left(v \omega_{z}\right)}{\partial y}+\frac{1}{R e}\left(\frac{\partial^{2} \omega_{z}}{\partial x^{2}}+\frac{\partial^{2} \omega_{z}}{\partial y^{2}}\right), \\
\frac{\partial^{2} v}{\partial x^{2}}+\frac{\partial^{2} v}{\partial y^{2}} & =-\frac{\partial \omega_{z}}{\partial x} \\
\frac{\partial u}{\partial x}+\frac{\partial v}{\partial y} & =0 .
\end{aligned}
$$

As condições de contorno são especificadas de acordo com o problema a ser considerado.

\subsection{Transição laminar-turbulento}

Um escoamento, inicialmente laminar, está sujeito a perturbações que podem transicionar para a turbulência. Essas perturbações podem ser geradas, por exemplo, pela presença de rugosidades na superfície do escoamento e estão associadas a fatores como a viscosidade do fluido e/ou a condição de não deslizamento. Mendonça (2003) cita que, em casos onde a amplitude dessas perturbações é grande, a transição à turbulência é abrupta e não linear. Neste caso, a transição é denominada bypass transition. No entanto, quando a magnitude dessas perturbações é infinitesimal, podem ser geradas ondas. Estas ondas são denominadas ondas de Tollmien-Schlichting.

O conceito de ondas de Tollmien-Schlichting surge pela necessidade de se justificar o paradoxo gerado pelo fato de uma camada limite laminar - teoricamente estável por não possuir nenhum ponto de inflexão ${ }^{1}$ - transicionar para a turbulência. Normalmente, essa transição é provocada por meio da instabilidade da camada limite laminar frente à perturbações e pode ser estudada, por exemplo, pela teoria de estabilidade linear. Resultados por ela previstos possibilitam uma alternativa viável para uma verificação do método numérico a ser proposto.

\subsubsection{Teoria de Estabilidade Linear}

A teoria de estabilidade linear (LST, do inglês Linear Stability Theory) propõe, segundo Schlichting (1979), a decomposição de um determinado escoamento em duas partes: escoamento principal e perturbação. De forma geral, a investigação de como essas perturbações comportam-se frente ao escoamento principal é o que caracteriza a LST.

Em duas dimensões, o escoamento principal é tomado como permanente, tendo $u$ e $v$ como componentes cartesianos da velocidade e $p$ como a pressão. Os componentes da perturbação são pequenas em relação ao escoamento principal e, em um regime transiente, são representados por $\tilde{u}, \tilde{v}$ e $\tilde{p}$. Tomando como incompressível o escoamento principal em questão, supõem-se que o mesmo satisfaz as equações de Navier-Stokes. Considera-se ainda que o escoamento principal é invariante na direção $x$,

\footnotetext{
${ }^{1}$ O teorema de Rayleigh, proposto em 1913, afirma que uma condição necessária para a ocorrência de instabilidade é a existência de um ponto de inflexão no perfil de velocidade.
} 
ou seja,

$$
u=u(y), \quad v=0 \quad \text { e } p=p(x, y)
$$

Assim, os componentes de velocidade do escoamento podem ser dados por:

$$
U=u+\tilde{u}, \quad V=\tilde{v}
$$

e a pressão,

$$
P=p+\tilde{p}
$$

Já que o escoamento principal é por hipótese, solução das equações de Navier-Stokes, as resultantes acima descritas também devem satisfazer esse sistema de equações. Schlichting (1979) ainda afirma que as perturbações devem ser pequenas no sentido de que termos não lineares possam ser desprezados quando comparados com os termos lineares.

Substituindo as equações (2.15) e (2.16) no sistema composto pelas equações de Navier-Stokes descrito por (2.3) a (2.5), obtém-se:

$$
\begin{aligned}
\frac{\partial \tilde{u}}{\partial t}+u \frac{\partial \tilde{u}}{\partial x}+\tilde{v} \frac{\partial u}{\partial y}+\left[\tilde{u} \frac{\partial \tilde{u}}{\partial x}+\tilde{v} \frac{\partial \tilde{u}}{\partial y}\right] & =-\frac{\partial \tilde{p}}{\partial x}+\frac{1}{R e}\left(\frac{\partial^{2} \tilde{u}}{\partial x^{2}}+\frac{\partial^{2} \tilde{u}}{\partial y^{2}}\right) \\
\frac{\partial \tilde{v}}{\partial t}+u \frac{\partial \tilde{v}}{\partial x}+\left[\tilde{u} \frac{\partial \tilde{v}}{\partial x}+\tilde{v} \frac{\partial \tilde{v}}{\partial y}\right] & =-\frac{\partial \tilde{p}}{\partial y}+\frac{1}{R e}\left(\frac{\partial^{2} \tilde{v}}{\partial x^{2}}+\frac{\partial^{2} \tilde{v}}{\partial y^{2}}\right) \\
\frac{\partial \tilde{u}}{\partial x}+\frac{\partial \tilde{v}}{\partial y} & =0
\end{aligned}
$$

em que $\tilde{u}=\tilde{u}(x, y, t), \tilde{v}=\tilde{v}(x, y, t)$ e $\tilde{p}=\tilde{p}(x, y, t)$.

Tomando como válida a hipótese associada à pequena magnitude das perturbações, é possível linearizar o sistema de equações acima, desprezando-se os termos entre colchetes. Sendo assim, o sistema simplificado dá-se por:

$$
\begin{aligned}
\frac{\partial \tilde{u}}{\partial t}+u \frac{\partial \tilde{u}}{\partial x}+\tilde{v} \frac{\partial u}{\partial y} & =-\frac{\partial \tilde{p}}{\partial x}+\frac{1}{R e}\left(\frac{\partial^{2} \tilde{u}}{\partial x^{2}}+\frac{\partial^{2} \tilde{u}}{\partial y^{2}}\right), \\
\frac{\partial \tilde{v}}{\partial t}+u \frac{\partial \tilde{v}}{\partial x} & =-\frac{\partial \tilde{p}}{\partial y}+\frac{1}{R e}\left(\frac{\partial^{2} \tilde{v}}{\partial x^{2}}+\frac{\partial^{2} \tilde{v}}{\partial y^{2}}\right), \\
\frac{\partial \tilde{u}}{\partial x}+\frac{\partial \tilde{v}}{\partial y} & =0 .
\end{aligned}
$$


Considerando um escoamento de Poiseuille, as condições de contorno são dadas por:

$$
\begin{aligned}
& \tilde{u}=\tilde{v}=0 \quad \text { para } y=0, \\
& \tilde{u}=\tilde{v}=0 \text { para } y=2 L \text {, }
\end{aligned}
$$

as quais correspondem à condição de não escorregamento e impermeabilidade nas paredes. A linearização do sistema de equações diferenciais parciais em questão possibilita a redução do mesmo a um sistema de equações diferenciais ordinárias. Para tanto, busca-se por meio do método de separação de variáveis, soluções na forma

$$
\begin{aligned}
& \tilde{u}_{1}(x, y, t)=\bar{u}(y) e^{i(\alpha x-\omega t)}, \\
& \tilde{v}_{1}(x, y, t)=\bar{v}(y) e^{i(\alpha x-\omega t)}, \\
& \tilde{p}_{1}(x, y, t)=\bar{p}(y) e^{i(\alpha x-\omega t)},
\end{aligned}
$$

sendo $i$ a unidade imaginária, $\alpha$ o número de onda na direção $x$ e $\omega$ a frequência com que as perturbações, de comprimento de onda $\lambda=\frac{2 \pi}{\alpha}$, propagam-se. Considera-se ainda $\bar{u}, \bar{v}$ e $\bar{p}$ como amplitudes das perturbações. Torna-se interessante ressaltar que, supondo que as equações (2.25) a (2.27) compõem uma solução para o sistema simplificado, os complexos conjugados

$$
\begin{aligned}
\tilde{u_{2}}(x, y, t) & =\bar{u}(y)^{*} e^{i\left(\alpha^{*} x-\omega^{*} t\right)}, \\
\tilde{v_{2}}(x, y, t) & =\bar{v}(y)^{*} e^{i\left(\alpha^{*} x-\omega^{*} t\right)}, \\
\tilde{p_{2}}(x, y, t) & =\bar{p}(y)^{*} e^{i\left(\alpha^{*} x-\omega^{*} t\right)},
\end{aligned}
$$

também compõem uma possível solução do sistema em questão. Uma vez que, almeja-se uma solução pertencente ao conjunto dos números reais, é razoável tomar como solução uma combinação linear das soluções anteriormente apresentadas. Assim,

$$
\begin{aligned}
\tilde{u}(x, y, t) & =\frac{1}{2}\left(\tilde{u}_{1}+\tilde{u}_{2}\right), \\
\tilde{v}(x, y, t) & =\frac{1}{2}\left(\tilde{v}_{1}+\tilde{v}_{2}\right), \\
\tilde{p}(x, y, t) & =\frac{1}{2}\left(\tilde{p}_{1}+\tilde{p}_{2}\right) .
\end{aligned}
$$

Substituindo as equações (2.31), (2.32) e (2.33) no sistema linearizado composto pelas equações (2.20), (2.21) e (2.22), obtém-se a expressão

$$
i \alpha \bar{u}+\frac{d \bar{v}}{d y}=0
$$


para a conservação da massa e, para as equações de quantidade de movimento:

$$
\begin{aligned}
-i \omega \bar{u}+i \alpha \bar{u} u+\bar{v} \frac{d u}{d y} & =-i \alpha \bar{p}+\frac{1}{R e}\left((i \alpha)^{2} \bar{u}+\frac{d^{2} \bar{u}}{d y^{2}}\right) \\
-i \omega \bar{v}+i \alpha u \bar{v} & =-\frac{d \bar{p}}{d y}+\frac{1}{R e}\left((i \alpha)^{2} \bar{v}+\frac{d^{2} \bar{v}}{d y^{2}}\right) .
\end{aligned}
$$

Considerando $c=\omega / \alpha$ como sendo a velocidade de onda, reescreve-se as equações acima em função das autofunções $\bar{u}, \bar{v}$ e $\bar{p}$,

$$
\begin{aligned}
i \alpha(u-c) \bar{u}+\frac{d u}{d y} \bar{v}+i \alpha \bar{p} & =\frac{1}{R e}\left(\frac{d^{2} \bar{u}}{d y^{2}}-\alpha^{2} \bar{u}\right), \\
i \alpha(u-c) \bar{v}+\frac{d \bar{p}}{d y} & =\frac{1}{R e}\left(\frac{d^{2} \bar{v}}{d y^{2}}-\alpha^{2} \bar{v}\right), \\
i \alpha \bar{u}+\frac{d \bar{v}}{d y} & =0 .
\end{aligned}
$$

Simplificações podem ser realizadas derivando-se a equação (2.37) em relação a $y$,

$$
i \alpha(u-c) \frac{d \bar{u}}{d y}+i \alpha \bar{u} \frac{d u}{d y}+\frac{d \bar{v}}{d y} \frac{d u}{d y}+\bar{v} \frac{d^{2} u}{d y^{2}}+i \alpha \frac{d \bar{p}}{d y}=\frac{1}{R e}\left(\frac{d^{3} \bar{u}}{d y^{3}}-\alpha^{2} \frac{d \bar{u}}{d y}\right),
$$

multiplicado-se (2.38) pela constante $i \alpha$

$$
-\alpha^{2}(u-c) \bar{v}+i \alpha \frac{d \bar{p}}{d y}=\frac{1}{R e}\left(i \alpha \frac{d^{2} \bar{v}}{d y^{2}}-i \alpha^{3} \bar{v}\right)
$$

e realizando a diferença entre as equações (2.40) e (2.41), a qual resulta em:

$$
\begin{aligned}
i \alpha(u-c) \frac{d \bar{u}}{d y} & +i \alpha \bar{u} \frac{d u}{d y}+\frac{d \bar{v}}{d y} \frac{d u}{d y}+\bar{v} \frac{d^{2} u}{d y^{2}}+\alpha^{2}(u-c) \bar{v} \\
& =\frac{1}{R e}\left(\frac{d^{3} \bar{u}}{d y^{3}}-\alpha^{2} \frac{d \bar{u}}{d y}+i \alpha^{3} \bar{v}-i \alpha \frac{d^{2} \bar{v}}{d y^{2}}\right) .
\end{aligned}
$$

Sendo a equação da continuidade, obtém-se:

$$
\begin{aligned}
i(u-c) \frac{d}{d y}\left(i \frac{d \bar{v}}{d y}\right) & +\bar{v} \frac{d^{2} u}{d y^{2}}+\alpha^{2}(u-c) \bar{v} \\
& =\frac{1}{R e}\left(\frac{d^{3}}{d y^{3}}\left(\frac{i}{\alpha} \frac{d \bar{v}}{d y}\right)-\alpha^{2} \frac{d}{d y}\left(\frac{i}{\alpha} \frac{d \bar{v}}{d y}\right)+i \alpha^{3} \bar{v}-i \alpha \frac{d^{2} \bar{v}}{d y^{2}}\right) .
\end{aligned}
$$

Multiplicando-se a equação acima por $\alpha$, uma equação diferencial ordinária de quarta ordem é obtida. A esta equação, definida por (2.44), dá-se o nome de equação de Orr-Sommerfeld.

$$
\alpha(u-c)\left(\bar{v}^{\prime \prime}-\alpha^{2} \bar{v}\right)-u^{\prime \prime} \alpha \bar{v}=-\frac{i}{R e}\left(\bar{v}^{I V}-2 \alpha^{2} \bar{v}^{\prime \prime}+\alpha^{4} \bar{v}\right)
$$


em que a $\left({ }^{\prime}\right)$ representa derivação em função de $y$.

\subsubsection{Análise espacial e temporal de instabilidades}

A equação (2.25), derivada no campo dos números complexos, possui a autofunção $\bar{u}$, e as constantes $\omega$ e $\alpha$, complexos. Neste sentido, expressa-se a solução do sistema como:

$$
\tilde{u}(x, y, t)=\bar{u}(y) e^{\left(-\alpha_{i} x+\omega_{i} t\right)} e^{i\left(\alpha_{r} x-\omega_{r} t\right)},
$$

em que os índices $r$ e $i$ representam componentes real e imaginária, respectivamente.

Observa-se que, quando $\omega$ é um número real e $\alpha$ é um número complexo, a amplitude da perturbação cresce na direção do escoamento médio em $x$. Neste sentido, a formulação em questão é denominada formulação espacial. Nota-se que os componentes $\omega_{r}, \alpha_{r}$ e $\alpha_{i}$ representam, respectivamente, a frequência, o número de onda e a taxa de amplificação espacial. Em contrapartida, na formulação denominada temporal, $\alpha$ é tomado como um número real e $\omega$ como sendo um número complexo. Para este caso, a amplitude da perturbação cresce diretamente em função do tempo. A taxa de amplificação, para esta formulação, é dada por $\omega_{i}$. Portanto, tem-se:

Tabela 2.1: Classificação de instabilidades.

\begin{tabular}{cccc}
\hline \hline Tipo de análise & taxa de amplificação & amplitude & classificação \\
\hline \multirow{2}{*}{ análise temporal } & $\omega_{i}<0$ & decresce & estável \\
& $\omega_{i}=0$ & constante & neutro \\
& $\omega_{i}>0$ & cresce & instável \\
& $\alpha_{i}<0$ & cresce & instável \\
análise espacial & $\alpha_{i}=0$ & constante & neutro \\
& $\alpha_{i}>0$ & decresce & estável \\
\hline \hline
\end{tabular}

No presente trabalho somente a análise espacial é utilizada.

\subsubsection{Solução numérica da equação de Orr-Sommerfeld}

A solução da equação de Orr-Sommerfeld corresponde a um problema de autovalor. A existência de uma solução, neste sentido, está diretamente vinculada aos valores de $\alpha, \omega$ e ao número de Reynolds $R e$, e depende do perfil de velocidade do escoamento base em questão. De maneira geral, a equação de Orr-Sommerfeld pode ser reescrita como

$$
\bar{v}^{I V}+A(y) \bar{v}^{\prime \prime}+B(y) \bar{v}=0
$$


em que:

$$
\begin{aligned}
& A(y)=-2 \alpha^{2}-i \operatorname{Re}(\alpha u-\omega), \\
& B(y)=\alpha^{4}+i \operatorname{Re} \alpha^{2}(\alpha u-\omega)+i \alpha \operatorname{Reu} .
\end{aligned}
$$

Reescrevendo essa equação na forma matricial $\bar{V}^{\prime}=M \bar{V}$, tem-se:

$$
\left[\begin{array}{c}
\bar{v}^{\prime} \\
\bar{v}^{\prime \prime} \\
\bar{v}^{\prime \prime \prime} \\
\bar{v}^{I V}
\end{array}\right]=\left[\begin{array}{cccc}
0 & 1 & 0 & 0 \\
0 & 0 & 1 & 0 \\
0 & 0 & 0 & 1 \\
-B(y) & 0 & -A(y) & 0
\end{array}\right]\left[\begin{array}{c}
\bar{v} \\
\bar{v}^{\prime} \\
\bar{v}^{\prime \prime} \\
\bar{v}^{\prime \prime \prime}
\end{array}\right]
$$

Inicia-se o cálculo pela parede inferior. Para $y=0$, uma combinação linear de soluções deve satisfazer as condições $\bar{v}=\bar{v}^{\prime}=0$. Assim, o vetor solução $\bar{V}=\left[\bar{v}, \bar{v}^{\prime}, \bar{v}^{\prime \prime}, \bar{v}^{\prime \prime \prime}\right]$ pode ser escrito por meio da base canônica

$$
\bar{V}_{(y=0)}=a_{1}\left[\begin{array}{l}
1 \\
0 \\
0 \\
0
\end{array}\right]+a_{2}\left[\begin{array}{l}
0 \\
1 \\
0 \\
0
\end{array}\right]+a_{3}\left[\begin{array}{l}
0 \\
0 \\
1 \\
0
\end{array}\right]+a_{4}\left[\begin{array}{l}
0 \\
0 \\
0 \\
1
\end{array}\right]
$$

onde é necessário que $a_{1}=a_{2}=0$, para que a condição de não escorregamento e impermeabilidade seja satisfeita. Tem-se então, para o contorno inferior:

$$
b_{1} \bar{V}_{I}+b_{2} \bar{V}_{I I}=a_{3}\left[\begin{array}{l}
0 \\
0 \\
1 \\
0
\end{array}\right]+a_{4}\left[\begin{array}{l}
0 \\
0 \\
0 \\
1
\end{array}\right]
$$

que na forma matricial é dada por:

$$
\left[\begin{array}{cccc}
\bar{v}_{I} & \bar{v}_{I}^{\prime} & \bar{v}_{I}^{\prime \prime} & \bar{v}_{I}^{\prime \prime \prime} \\
\bar{v}_{I I} & \bar{v}_{I I}^{\prime} & \bar{v}_{I I}^{\prime \prime} & \bar{v}_{I I}^{\prime \prime \prime} \\
0 & 0 & -1 & 0 \\
0 & 0 & 0 & -1
\end{array}\right]\left[\begin{array}{l}
b_{1} \\
b_{2} \\
a_{3} \\
a_{4}
\end{array}\right]=\left[\begin{array}{l}
0 \\
0 \\
0 \\
0
\end{array}\right]
$$

Para o sistema linear acima admitir uma solução não trivial, faz-se necessário que:

$$
\left|\begin{array}{cccc}
\bar{v}_{I} & \bar{v}_{I}^{\prime} & \bar{v}_{I}^{\prime \prime} & \bar{v}_{I}^{\prime \prime \prime} \\
\bar{v}_{I I} & \bar{v}_{I I}^{\prime} & \bar{v}_{I I}^{\prime \prime} & \bar{v}_{I I}^{\prime \prime \prime} \\
0 & 0 & -1 & 0 \\
0 & 0 & 0 & -1
\end{array}\right|=0
$$


Um Shooting method, implementado por Mendonça (2003), é utilizado para resolver a equação de Orr-Sommerfeld. Para tanto, os seguintes passos são realizados:

a. Considera-se valores iniciais para os autovalores $\alpha, \omega$ e $R e$;

b. Integra-se a equação de Orr-Sommerfeld, por meio de um método de Runge-Kutta de quarta ordem, partindo-se do contorno inferior;

c. Verifica-se se a condição de contorno no contorno superior é satisfeita, ou seja, se a restrição (2.53) é respeitada;

d. Altera-se o valor de $\omega$, de forma que o mesmo esteja próximo do valor anterior;

e. Integra-se novamente a equação. Verifica-se se a restrição é satisfeita ${ }^{2}$;

f. Caso a restrição não seja respeitada, interpola-se $\omega$ por meio das últimas duas aproximações. Neste ponto, o algoritmo retorna ao item (e.).

Uma vez que os autovalores da equação de Orr-Sommerfeld apresentam distintas ordens de magnitude, faz-se necessário, segundo Mendonça (2003), garantir a independência linear da solução. Para tanto, adota-se o procedimento denominado ortonormalização de Gram-Schmidt sobre os vetores solução.

\subsection{Método numérico}

\subsubsection{Introdução de perturbações}

Em concordância com estudos de Fasel et al. (1990), a introdução de perturbações temporais é realizada através de injeção e sucção de massa nas paredes. Justifica-se a escolha devido a questões de estabilidade e maior coerência com resultados experimentais e numéricos ${ }^{3}$.

Para tanto, considerando o tempo inicial $t=0$, assume-se que o escoamento é não perturbado. A partir do tempo $t+\Delta t$, as perturbações são inseridas em uma determinada região dos contornos, delimitada pelos extremos $x_{1}$ e $x_{2}$. A perturbação é representada por meio de uma função $p(x)$ de $9^{o}$ grau para a velocidade $v$, na qual:

$$
\begin{aligned}
v & =A p(x) \operatorname{sen}(\omega t) \quad x_{1}<x<x_{2} \\
& \mathrm{e} \\
v & =0 \text { para } x \leq x_{1} \text { ou } x \geq x_{2} .
\end{aligned}
$$

\footnotetext{
${ }^{2} \mathrm{O}$ uso dos valores de $\omega^{k-1}$ e $\omega^{k}$, no sentido de se estimar $\omega^{k+1}$, é caracterizado pelo método das Secantes.

${ }^{3}$ Como alternativa, de acordo com os trabalhos de Souza (2003), há possibilidade dessas perturbações serem inseridas na entrada do escoamento.
} 
onde $A$ representa uma constante, $\omega$ é a frequência e $t$, o tempo. Os coeficientes do polinômio são escolhidos convenientemente objetivando anular o valor da função e das suas primeira e segunda derivadas nos pontos $x_{1}$ e $x_{2}$.

\subsubsection{Esquemas multigrid}

Na tentativa de explicitar a característica de que métodos iterativos reduzem efetivamente erros associados às altas frequências, considera-se a equação de Poisson bidimensional, em um domínio retangular de dimensões $0 \leq x \leq 1$ e $0 \leq y \leq 1$, dada por

$$
\nabla^{2} v=s
$$

sujeita à condição de contorno

$$
v(x, y)=\operatorname{sen}(x) \cos (y)
$$

E o termo fonte definido,

$$
s(x, y)=-2 \operatorname{sen}(x) \cos (y) .
$$

A solução analítica da equação (2.56), sujeita à (2.57) e (2.58) e representada pela figura 2.1(a), é dada por $v(x, y)=\operatorname{sen}(x) \cos (y)$. Aproxima-se a equação diferencial (2.56) por meio de diferenças finitas centradas de $2^{\mathrm{a}}$ ordem, considerando $i \max =j \max =100$ como o número de pontos discretizados nas direções $x$ e $y$. O sistema linear obtido por meio dessa aproximação pode ser resolvido numericamente, por exemplo, pelo método de Jacobi subrelaxado por um fator $w=0.9$. Considera-se então, o chute inicial dado como:

$$
v_{0}(x, y)=\operatorname{sen}(2 \pi x) \cos (80 \pi x)
$$

Ressalta-se que a escolha do chute inicial, dado por (2.59), contempla um modo de baixa $(n=2)$ e alta $(n=80)$ frequência ${ }^{4}$. O gráfico associado à função (2.59) é representado pela figura 2.1(b).

As figuras 2.2(a), 2.2(b), 2.2(c) e 2.2(d) apresentam o comportamento do módulo do erro após 0, 1, 25 e 1000 iterações, respectivamente. Nota-se claramente que, com um pequeno número de iterações do método de Jacobi subrelaxado, os modos de alta frequência do erro são drasticamente reduzidos, prevalecendo os modos associados às baixas frequências. Tal fato justifica, por exemplo, o fato do método de Jacobi subrelaxado ser considerado como um suavizador.

A estratégia referente a utilização de um conjunto diferente de malhas para um método multigrid é justificada devido ao fato de que a linha de divisão entre altas e baixas frequências do erro varia

\footnotetext{
${ }^{4}$ Segundo Briggs et al. (2000), os números de onda pertencentes a parte inferior do espectro, ou seja, pertencentes ao intervalo $1 \leq k \leq \frac{n}{2}$ são denominados de modos suaves ou modos de baixa frequência. Além disso, os números de onda que pertencem a parte superior do espectro, ou seja, $\frac{n}{2} \leq k \leq n-1$ são denominados de modos oscilatórios ou modos de alta frequência.
} 


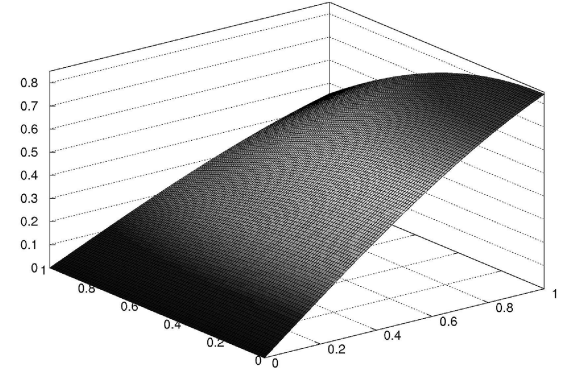

(a) Solução analítica

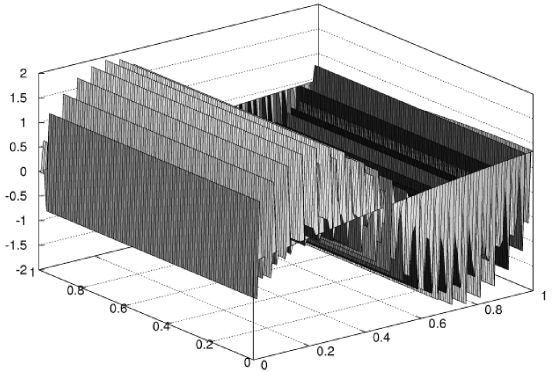

(b) Chute inicial

Figura 2.1: 2.1(a) Solução analítica da função $v$ e 2.1(b) Chute inicial utilizado.

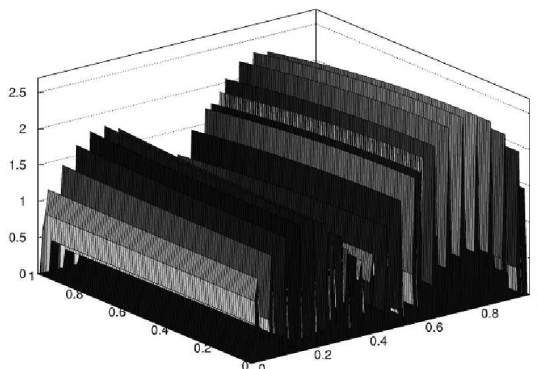

(a) inicial

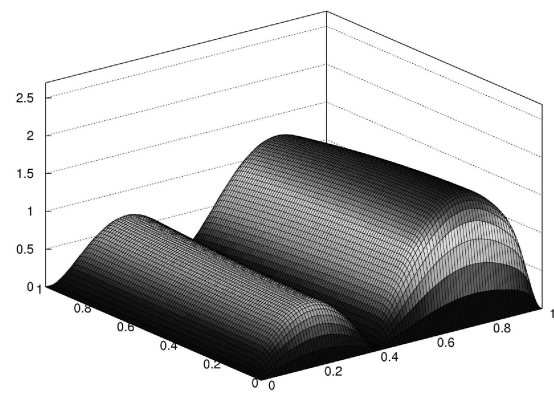

(c) 25 iterações

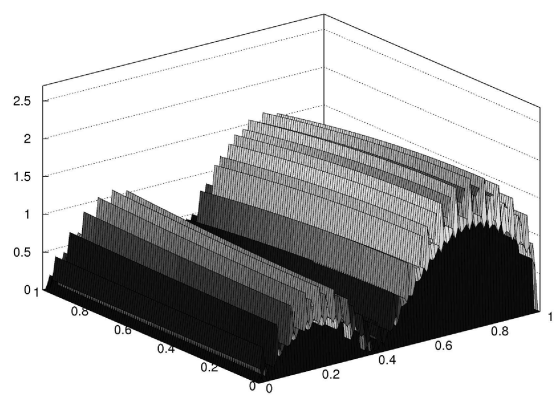

(b) 1 iteração

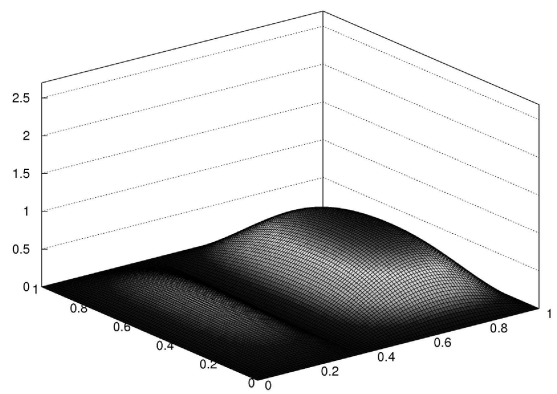

(d) 1000 iterações

Figura 2.2: Comportamento do módulo do erro após: (a) nenhuma iteração; (b) 1 iteração; (c) 25 iterações; (d) 1000 iterações.

de acordo com o espaçamento. De forma simplista, baixas frequências associadas a uma malha de espaçamento $h$ podem ser consideradas altas em malhas com maior espaçamento. A consequência direta desse fato é o aumento da taxa de convergência, como citado anteriormente, uma vez que há redução do erro em todas as frequências.

São analisados/implementados dois métodos multigrid: um esquema de correção - CS, do inglês 
Correction Scheme e um esquema de aproximação total - FAS, do inglês Full Aproximation Scheme. Para os casos considerados, o sistema linear foi resolvido por meio de um ciclo $\mathrm{V}$ composto por 4 níveis representado pela figura 2.3, onde $S$ representa a aplicação do suavizador (e/ou método iterativo), $R$ representa a operação de restrição e $P$, a operação de prolongamento. Além disso, $h$ e $8 h$ representam os níveis mais e menos refinados, respectivamente. Uma vez que objetiva-se a resolução de um problema elíptico opta-se, de acordo com os trabalhos de Brandt (1977), por uma razão de engrossamento igual a 2 .

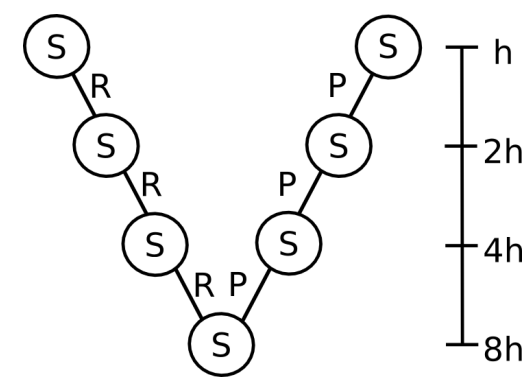

Figura 2.3: Esquema multigrid - Ciclo V

\subsubsection{Esquema de correção CS}

Seja a equação de Poisson bidimensional

$$
\frac{\partial^{2} v}{\partial x^{2}}+\frac{\partial^{2} v}{\partial y^{2}}=s
$$

em que $v$ representa a função a ser obtida e $s$ o termo fonte da equação.

$\mathrm{O}$ sistema linear associado à discretização dessa equação em cada malha de um ciclo $\mathrm{V}$, composto por quatro níveis e representado pela figura 2.4, é resolvido por meio de métodos iterativos. As constantes $N_{1}$ e $N_{2}$ representam o número de iterações aplicado em um determinado nível.

O método de Jacobi por linhas é aplicado a todos os níveis, exceto ao referente a malha mais grossa, na qual é aplicado o método SOR por linhas. Para o método de Jacobi é utilizado um fator de relaxação de $r_{1}$. No caso do método SOR, o fator utilizado é de $r_{2}$. Vale ressaltar que não há aplicação de métodos iterativos no ramo direito do ciclo V, como explicitado na figura 2.4.

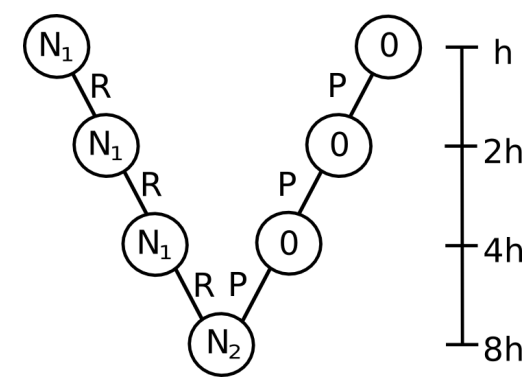

Figura 2.4: Esquema de correção CS - Ciclo V 
Tomando como referência as informações apresentadas, o algoritmo associado ao esquema CS pode ser descrito da seguinte forma:

Partindo da malha mais refinada - considerando um espaçamento $h$ - são realizadas $N_{1}$ iterações visando a solução da equação de Poisson:

$$
\nabla^{2} v_{h}=s_{h}
$$

De posse do valor obtido após essas $N_{1}$ iterações, obtém-se o resíduo $\left(d_{h}\right)$, por meio de:

$$
d_{h}=s_{h}-\nabla^{2} v_{h}
$$

Para o caso do esquema CS, a transferência de valores de um nível mais refinado $(h)$, para outro, menos refinado $(2 h)$, é dada por uma operação denominada restrição. Por meio dessa operação, os valores associados ao resíduo são transmitidos para a malha mais grossa, através de uma ponderação. Os valores obtidos por meio dessa ponderação, na malha mais grossa, são então definidos como o termo fonte da equação de Poisson associada ao erro ${ }^{5}$.

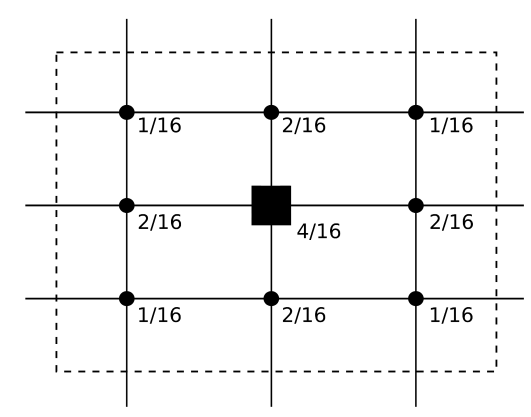

Figura 2.5: Operação FW

Essa operação é denominada ponderação completa - FW, do inglês Full Weight. A expressão abaixo exprime essa operação, a qual é ilustrada pela figura 2.5.

$$
d_{h} \Rightarrow s_{2 h} .(F W)
$$

Tendo como referência a figura 2.5, apenas o elemento identificado pelo retângulo central é transmitido, de forma ponderada, para a malha menos refinada. A contribuição de cada ponto é apresentada ao lado do mesmo.

Considerando agora a malha representada por $(2 h)$, faz-se novamente $N_{1}$ iterações, sendo:

$$
\nabla^{2} v_{2 h}=s_{2 h}
$$

Nota-se que a solução do sistema linear nessa malha objetiva a obtenção do erro associado às

\footnotetext{
${ }^{5}$ Vale ressaltar que, para um dado sistema linear, o erro pode ser definido como $e=\bar{v}-v$, onde $v$ é a solução numérica e $\bar{v}$ é a solução analítica. Assim, $A e=A \bar{v}-A v=s-A v=d$.
} 
baixas frequências decorrentes da solução do sistema na malha de espaçamento $(h)$. Estima-se como nulo o erro inicial. De forma análoga a apresentada anteriormente, o resíduo $d_{2 h}$ é obtido por meio de:

$$
d_{2 h}=s_{2 h}-\nabla^{2} v_{2 h}
$$

Realiza-se sucessivamente os passos apresentados até que se alcance o nível menos refinado (8h). Nesta malha, especificamente, são realizadas $N_{2}$ iterações com o método SOR por linhas.

O retorno a um nível mais refinado dá-se através de uma interpolação bilinear e posterior correção do valor da solução, onde:

$$
\begin{aligned}
& v_{8 h} \Rightarrow \operatorname{corr}_{4 h}, \\
& v_{4 h} \Leftarrow v_{4 h}+\operatorname{corr}_{4 h} .
\end{aligned}
$$

Ressalta-se que a interpolação deve ser aplicada até que se alcance o nível de espaçamento $(h)$. A interpolação bilinear é representada pela figura 2.6.

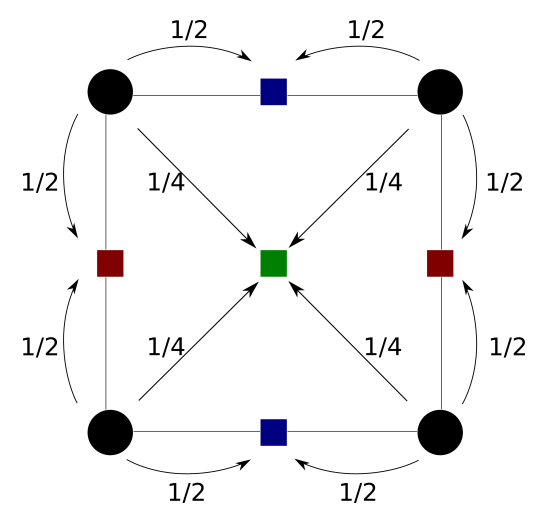

Figura 2.6: Interpolação bilinear

Atinge-se o critério de parada quando o resíduo obtido na malha mais fina for menor do que uma dada tolerância de referência. Caso contrário, o ciclo é novamente aplicado.

\subsubsection{Esquema de aproximação total FAS - Full Aproximation Scheme}

Considera-se novamente a equação de Poisson dada pela equação (2.60) e o ciclo V representado pela figura 2.7.

A resolução dessa equação, ao contrário do método anterior, é realizada apenas utilizando o método SOR por linhas. Especificamente, aplica-se o método de Gauss-Seidel após a operação de prolongamento. Nos demais casos, o fator de relaxação utilizado é dado por $r_{2}$. O algoritmo associado ao esquema FAS pode ser descrito da seguinte forma:

Partindo da malha mais refinada - considerando um espaçamento $h$ - são realizadas $N_{1}$ iterações 


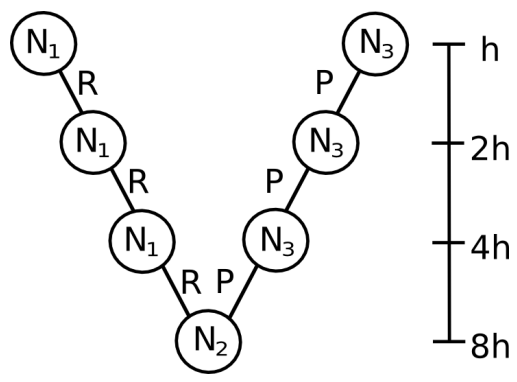

Figura 2.7: Método multigrid FAS - Ciclo V

visando a solução da equação de Poisson:

$$
\nabla^{2} v_{h}=s_{h}
$$

De posse do valor obtido após essas $N_{1}$ iterações, obtém-se o resíduo $\left(d_{h}\right)$, por meio de:

$$
d_{h}=s_{h}-\nabla^{2} v_{h}
$$

Da mesma forma como ocorre no método CS, a transição de valores de um nível mais refinado $(h)$, para outro, menos refinado $(2 h)$, é dada pela operação de restrição, em que o resíduo é transmitido por meio de uma ponderação (FW). Porém, faz-se também necessário transmitir os valores associados à solução intermediária para a malha mais grossa. Nestes casos, não há aplicação de nenhum tipo de ponderação e os valores são transmitidos diretamente da malha mais fina para a mais grossa. Essa operação, representada pela figura 2.8, é denominada restrição por injeção direta - SI, do inglês Straight Injection. A expressão abaixo exprime-a, juntamente com a operação FW.

$$
\begin{aligned}
& v_{h} \Rightarrow v_{2 h}(S I), \\
& d_{h} \Rightarrow d_{2 h}(F W) .
\end{aligned}
$$

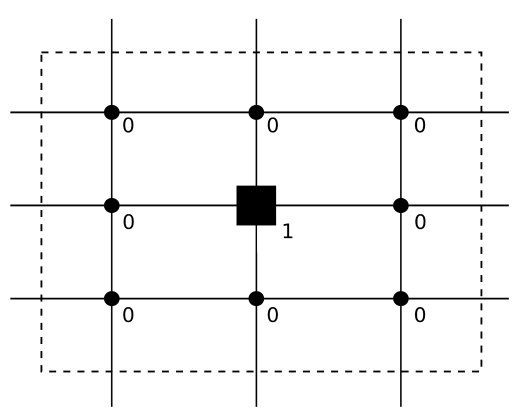

Figura 2.8: Operação SI

Tomando como referência os valores transmitidos, o termo fonte é tomado como:

$$
s_{2 h}=d_{2 h}+\nabla^{2} v_{2 h}
$$


Considerando a malha representada por $(2 h)$, faz-se novamente $N_{1}$ iterações, sendo:

$$
\nabla^{2} v_{2 h}=s_{2 h}
$$

Neste caso, o resultado obtido leva em consideração além do erro associado a malha $(h)$, a solução do problema de Poisson na malha $(2 h)$. Isso se dá uma vez que o termo fonte referente à malha $(2 h)$ é obtido considerando informações obtidas por meio das operações SI e FW. O valor inicial adotado, neste sentido, provém diretamente da operação SI. Estima-se o valor do resíduo $d_{2 h}$ como:

$$
d_{2 h}=s_{2 h}-\nabla^{2} v_{2 h}
$$

Realiza-se os passos apresentados até que se alcance o nível menos refinado (8h). Nesta malha, especificamente, são realizadas $N_{2}$ iterações.

Na sequência, gera-se a correção referente à malha mais grossa, dada por:

$$
\operatorname{corr}_{8 h}=v_{8 h}^{n}-v_{8 h}^{\prime}
$$

em que $v_{8 h}^{\prime}$ representa a aproximação gerada por meio da operação de restrição e $v_{8 h}^{n}$ a aproximação mais recentemente calculada.

O retorno à um nível mais refinado dá-se através de uma interpolação bilinear, onde:

$$
\begin{aligned}
\operatorname{corr}_{8 h} & \Rightarrow \operatorname{corr}_{4 h} ; \\
v_{4 h} & \Leftarrow v_{4 h}+\operatorname{corr}_{4 h} .
\end{aligned}
$$

Finalmente, realiza-se mais $N_{3}$ iterações, aplicando-se o método de Gauss-Seidel:

$$
\nabla^{2} v_{4 h}=s_{4 h}
$$

Para todos os casos considerados, $N_{3}=1$.

O ciclo é finalizado após a aplicação da interpolação bilinear referente à malha com espaçamento $(2 h)$. O número de ciclos necessário para que a solução da equação seja, de fato, obtida, está associado ao resíduo obtido na malha mais fina. Se o valor obtido for menor do que uma dada tolerância de referência, o algoritmo é finalizado.

\subsubsection{Aproximações utilizadas}

No sentido de estimar as derivadas associadas à equação de Poisson, por meio de diferenças finitas, são utilizadas as seguintes aproximações para a função $v$.

1. Na direção $x$ : 
- $i=2$ :

$$
\left.\frac{\partial^{2} v}{\partial x^{2}}\right|_{2, j}=\frac{10 v_{1, j}-15 v_{2, j}-4 v_{3, j}+14 v_{4, j}-6 v_{5, j}+v_{6, j}}{12 \Delta x^{2}}+O\left(\Delta x^{4}\right)
$$

- $3 \leq i \leq \operatorname{imax}-2$ :

$$
\left.\frac{\partial^{2} v}{\partial x^{2}}\right|_{i, j}=\frac{-v_{i-2, j}+16 v_{i-1, j}-30 v_{i, j}+16 v_{i+1, j}-v_{i+2, j}}{12 \Delta x^{2}}+O\left(\Delta x^{4}\right) ;
$$

- $i=i \max -1$ : uma aproximação similar a $i=2$ é utilizada.

2. Na direção $y$ :

- $j=2$ :

$$
\left.\frac{4}{5} \frac{\partial^{2} v}{\partial y^{2}}\right|_{i, 2}+\left.\frac{1}{5} \frac{\partial^{2} v}{\partial y^{2}}\right|_{i, 3}=\frac{254 v_{i, 1}-432 v_{i, 2}+162 v_{i, 3}+16 v_{i, 4}}{180 \Delta y^{2}}+O\left(\Delta y^{5}\right)
$$

- $3 \leq j \leq j \max -2$ :

$$
\begin{aligned}
& \left.\frac{2}{15} \frac{\partial^{2} v}{\partial y^{2}}\right|_{i, j-1}+\left.\frac{11}{15} \frac{\partial^{2} v}{\partial y^{2}}\right|_{i, j}+\left.\frac{2}{15} \frac{\partial^{2} v}{\partial y^{2}}\right|_{i, j+1}= \\
& \frac{3 v_{i, j-2}+48 v_{i, j-1}-102 v_{i, j}+48 v_{i, j+1}+3 v_{i, j+2}}{60 \Delta y^{2}}+O\left(\Delta y^{6}\right)
\end{aligned}
$$

- $j=j \max -1$ : uma aproximação similar a $j=2$ é utilizada.

3. Na direção $y$, para casos onde há condição de contorno do tipo Neumann no contorno superior:

- $j=j \max -1$ :

$$
\frac{\partial^{2} v}{\partial^{2} y}=\frac{v_{i, j \max -2}-2 v_{i, j \max -1}+v_{i, j \max }}{\Delta y^{2}}+O\left(\Delta y^{2}\right)
$$

- $j=j \max :$

$$
\frac{\partial^{2} v}{\partial^{2} y}=-\frac{v_{i, j \max -2}+8 v_{i, j \max -1}-7 v_{i, j \max }}{2 \Delta y^{2}}+3 \frac{\partial v}{\partial y}+O\left(\Delta y^{2}\right) .
$$

Ressalta-se que as constantes $\Delta x$ e $\Delta y$ representam os espaçamentos nas direções $x$ e $y$, respectivamente. Busca-se a resolução da equação de Poisson, em um domínio retangular, em que imax e jmax representam o número máximo de pontos da discretização na direção $x$ e $y$, respectivamente.

\subsubsection{Derivadas Temporais}

O avanço temporal dá-se por meio da aplicação de um método Runge-Kutta clássico de quarta ordem de precisão, citado por Ferziger e Peric (1997). O método Runge-Kutta empregado é do 
tipo Previsor-Corretor onde, os métodos de Euler explícito e implícito são utilizados como previsor e corretor (respectivamente) para um avanço temporal de $t$ a $t+\frac{\Delta t}{2}$. O avanço para $t+\Delta t$ dá-se pela aplicação de um previsor baseado na regra do ponto médio e um corretor baseado na regra de Simpson. Os quatro passos do método são descritos como segue:

$$
\begin{aligned}
\omega_{z}^{\left(n+\frac{1}{2}\right)^{*}}= & \omega_{z}^{n}+\frac{\Delta t}{2} g\left(t^{n}, \omega_{z}^{n}\right), \\
\omega_{z}^{\left(n+\frac{1}{2}\right)^{* *}}= & \omega_{z}^{n}+\frac{\Delta t}{2} g\left(t^{n+\frac{1}{2}}, \omega_{z}^{\left(n+\frac{1}{2}\right)^{*}}\right), \\
\omega_{z}^{(n+1)^{*}}= & \omega_{z}^{n}+\Delta t g\left(t^{n+\frac{1}{2}}, \omega_{z}^{\left(n+\frac{1}{2}\right)^{* *}}\right), \\
\omega_{z}^{n+1}= & \omega_{z}^{n}+\frac{\Delta t}{6}\left[g\left(t^{n}, \omega_{z}^{n}\right)+2 g\left(t^{n+\frac{1}{2}}, \omega_{z}^{\left(n+\frac{1}{2}\right)^{*}}\right)\right. \\
& \left.+2 g\left(t^{n+\frac{1}{2}}, \omega_{z}^{\left(n+\frac{1}{2}\right)^{* *}}\right)+g\left(t^{n+1}, \omega_{z}^{(n+1)^{*}}\right)\right],
\end{aligned}
$$

onde $g\left(t, \omega_{z}\right)=-\frac{\partial\left(u \omega_{z}\right)}{\partial x}-\frac{\partial\left(v \omega_{z}\right)}{\partial y}+\frac{1}{R e}\left(\frac{\partial^{2} \omega_{z}}{\partial x^{2}}+\frac{\partial^{2} \omega_{z}}{\partial y^{2}}\right)$.

\subsubsection{Aproximação numérica de derivadas espaciais}

O uso de diferenças finitas compactas para estimar as derivadas primeira e segunda de uma função $f$ torna necessária a resolução de um sistema linear tridiagonal, para $6^{a}$ ordem de precisão. Para o cálculo da aproximação referente à primeira derivada, tem-se:

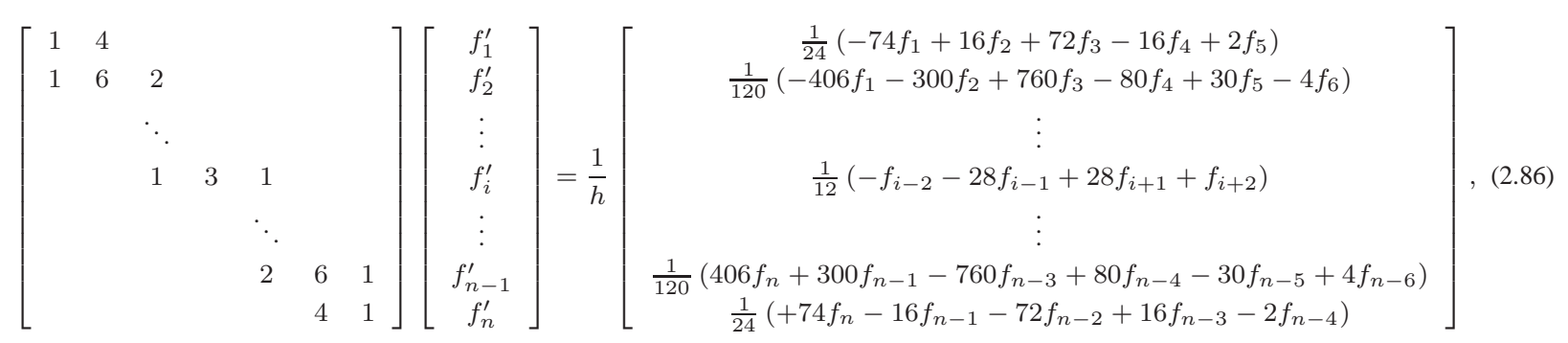

em que $h$ representa o espaçamento, $n$ é o número de pontos discretizados. De forma similar, o cálculo da segunda derivada resulta no sistema:

$$
\left[\begin{array}{ccccccc}
13 & 137 & & & & & \\
1 & 12 & 3 & & & & \\
& & \ddots & & & & \\
& & 2 & 11 & 2 & & \\
& & & & \ddots & & \\
& & & & 3 & 12 & 1 \\
& & & & & 137 & 13
\end{array}\right]\left[\begin{array}{c}
f_{1}^{\prime \prime} \\
f_{2}^{\prime \prime} \\
\vdots \\
f_{i}^{\prime \prime} \\
\vdots \\
f_{n-1}^{\prime \prime} \\
f_{n}^{\prime \prime}
\end{array}\right]=
$$




$$
=\frac{1}{h^{2}}\left[\begin{array}{c}
\frac{1}{60}\left(9775 f_{1}-20285 f_{2}+11170 f_{3}-550 f_{4}-145 f_{5}+36 f_{6}\right) \\
\frac{1}{360}\left(4834 f_{1}-8424 f_{2}+1890 f_{3}+2320 f_{4}-810 f_{5}+216 f_{6}-26 f_{7}\right) \\
\vdots \\
\frac{1}{4}\left(3 f_{i-2}+48 f_{i-1}-102 f_{i}+48 f_{i+1}+3 f_{i+2}\right) \\
\vdots \\
\frac{1}{360}\left(4834 f_{n}-8424 f_{n-1}+1890 f_{n-2}+2320 f_{n-3}-810 f_{n-4}+216 f_{n-5}-26 f_{n-6}\right) \\
\frac{1}{60}\left(9775 f_{n}-20285 f_{n-1}+11170 f_{n-2}-550 f_{n-3}-145 f_{n-4}+36 f_{n-5}\right)
\end{array}\right] .
$$

\subsubsection{Amortecimento de reflexões na entrada do escoamento}

Visando evitar possíveis reflexões, uma zona de amortecimento próxima à entrada é adotada e os componentes de vorticidade são ponderados por meio de uma função da forma:

$$
f_{1}(x)=f_{1}(\epsilon)=6 \epsilon^{5}-15 \epsilon^{4}+10 \epsilon^{3},
$$

em que $\epsilon$ é definida como uma função dependente dos pontos de discretização na direção longitudinal. A função $\epsilon$ é definida como:

$$
\epsilon=\frac{i-1}{i_{1}-1}
$$

onde $1 \leq i \leq i_{1}$. Assim, a função $f_{1}(x)$ é aplicada, no intervalo em questão, como segue:

$$
\omega_{k}(x, y, t)=f_{1}(x) \omega_{z}^{*}(x, y, t)
$$

Ressalta-se que $\omega_{z}^{*}(x, y, t)$ representa o componente de vorticidade que sai do integrador temporal e $\omega_{z}(x, y, t)$ é o valor do componente obtido após a aplicação do amortecimento.

\subsubsection{Relaminarização do escoamento}

Assim como ocorre na região de entrada do escoamento, efeitos não físicos associados à reflexão numérica de perturbações convectivas, na região de saída do escoamento, podem comprometer a qualidade dos resultados numéricos. Fundamento pelos trabalhos de Kloker (1998) e Meitz (1996), Souza (2003) adotou uma técnica de relaminarização no sentido de eliminar tais efeitos de reflexão de onda.

A técnica consiste em, considerando um intervalo próximo à saída do escoamento, impor que as perturbações dos componentes de vorticidade decaiam a zero. Neste sentido, considerando um intervalo $x_{3}<x<x_{4}$ em correspondência com os pontos de malha $i_{3}$ e $i_{4}$, utiliza-se uma função $f_{2}$, a qual varia de $f_{2}\left(x_{3}\right)=1$ até $f_{2}\left(x_{4}\right)=0$. Assim,

$$
\omega_{z}(x, y, t)=f_{2}(x) \omega_{z}^{*}(x, y, t)
$$

para todo o intervalo anteriormente definido. De forma análoga a subseção anterior, vale ressaltar que $\omega_{z}^{*}(x, y, t)$ representa o componente de vorticidade que sai do integrador temporal e $\omega_{z}(x, y, t)$ é 
tomado como o valor obtido após a relaminarização.

Kloker (1998) utiliza um polinônimo de $5^{a}$ ordem para a função $f_{2}(x)$, onde

$$
f_{2}(x)=f_{2}(\epsilon)=1-6 \epsilon^{5}+15 \epsilon^{4}+10 \epsilon^{3},
$$

tal que $\epsilon=\left(i-i_{3}\right) /\left(i_{4}-i_{3}\right)$, para todo $i_{3} \leq i \leq i_{4}$.

Além disso, Meitz (1996) verificou os efeitos de se aplicar a técnica de relaminarização $n$ vezes, a cada passo do integrador temporal. Ou seja,

$$
\omega_{z}(x, y, t)=f_{2}^{n}(x) \omega_{z}^{*}(x, y, t)
$$

Testes realizados por Meitz (1996), voltados à aplicação da equação (2.93) a uma função $\omega_{k}(x, y, t)$ constante, com $n=1,50,100$ e 150, mostraram que a repetida aplicação da função $f_{2}(x)$ gera um decaimento brusco. Este decaimento pode causar reflexões de onda na região de relaminarização. Como forma de evitar este comportamento brusco, Meitz (1996) propôs a utilização de uma função $f_{3}(x)$ na forma:

$$
f_{3}(x)=f_{3}(\epsilon)=e^{-\frac{\epsilon^{4}}{10}}\left(1-\epsilon^{50}\right)^{4} .
$$

No presente trabalho, opta-se pela utilização da função proposta por Meitz (1996). Souza (2003) ainda ressalta que, para o bom funcionamento da técnica, as seguintes restrições devem ser respeitadas:

$$
\begin{aligned}
i_{4}-i_{3} & \geq 2 \lambda, \\
i_{\max }-i_{4} & \geq \lambda,
\end{aligned}
$$

sendo $\lambda$ um comprimento de onda de Tollmien-Schlichting.

\subsubsection{Cálculo da vorticidade na parede}

Obtidas as soluções numéricas da equação de Poisson e da continuidade, o cálculo da vorticidade em $y=0$ ou $y=2 L$ pode ser realizado, segundo Souza (2003), efetuando-se os seguintes passos:

a. Calcula-se:

$$
J=\frac{\partial^{2} v}{\partial x^{2}}+\frac{\partial^{2} v}{\partial y^{2}}
$$

Observa-se que a segunda derivada na direção $x$, no intervalo entre $x_{1}$ e $x_{2}$, é obtida (analiticamente) considerando a mesma função utilizada para a inserção das ondas de Tollmien-Schlichting. Além disso, toma-se como nula a derivada nos demais pontos. Na direção $y$, a aproxima- 
ção explícita e descentrada abaixo apresentada é utilizada.

$$
\begin{aligned}
\left.\frac{\partial^{2} v}{\partial y^{2}}\right|_{y=0} & =\frac{1}{18 \Delta y^{2}}\left[-134.89 v_{1}+216 v_{2}-135 v_{3}+80 v_{4}-\right. \\
& \left.-33.75 v_{5}+8.64 v_{6}-v_{7}\right]+O\left(\Delta y^{6}\right)
\end{aligned}
$$

Em $y=2 L$, uma aproximação similar é empregada. Essas aproximações reforçam o fato de que $\left.\frac{\partial^{2} v}{\partial y^{2}}\right|_{y=\text { parede }}=0$.

b. Tendo os valores do laplaciano da velocidade, o componente de vorticidade é então obtido por meio da equação:

$$
\frac{\partial \omega_{z}}{\partial x}=-J
$$

As aproximações numéricas empregadas, também propostas por Souza (2003), são:

$$
\begin{aligned}
\text { para } i & =2 \text { : } \\
\omega_{z_{2}} & =\frac{1}{720}\left[\Delta x\left(251 \omega_{z_{1}}^{\prime}+646 \omega_{z_{2}}^{\prime}-264 \omega_{z_{3}}^{\prime}+106 \omega_{z_{4}}^{\prime}-19 \omega_{z_{5}}^{\prime}\right)+720 \omega_{z_{1}}\right]+O\left(\frac{3}{160} \Delta x^{6}\right) ;
\end{aligned}
$$

para $3 \leq i \leq i \max -2$ :

$$
\omega_{z_{i}}=\frac{1}{57}\left[\Delta x\left(10 \omega_{z_{i-1}}^{\prime}+57 \omega_{z_{i-1}}^{\prime}+24 \omega_{z_{i}}^{\prime}-\omega_{z_{i-1}}^{\prime}\right)+33 \omega_{z_{i-2}}+24 \omega_{z_{i-1}}\right]+O\left(\frac{11}{3420} \Delta x^{6}\right)
$$

para $i=i \max -1$ uma aproximação similar a $i=2$ é utilizada.

\subsubsection{Filtragem Espacial}

Souza (2003) em sua tese, estuda duas técnicas de filtragem no sentido de eliminar oscilações. A primeira, proposta por Kloker (1998), utiliza alternadamente esquemas descentrados compactos de $6^{a}$ ordem a montante e a juzante, em cada passo do método de Runge-Kutta. Ressalta-se que o esquema descentrado aplicado no último passo do integrador é novamente aplicado no primeiro passo do integrador no tempo posterior. A segunda estratégia, a qual foi de fato adotada, decorre de Lele (1992). Dentre os diversos métodos compactos de filtragem propostos pelo autor, opta-se pelo uso de um método compacto de $4^{a}$ ordem. A filtragem é aplicada ao final de cada iteração temporal e consiste em recalcular a distribuição dos componentes de vorticidade por meio de um sistema pentadiagonal, 
apresentado por Souza (2003):

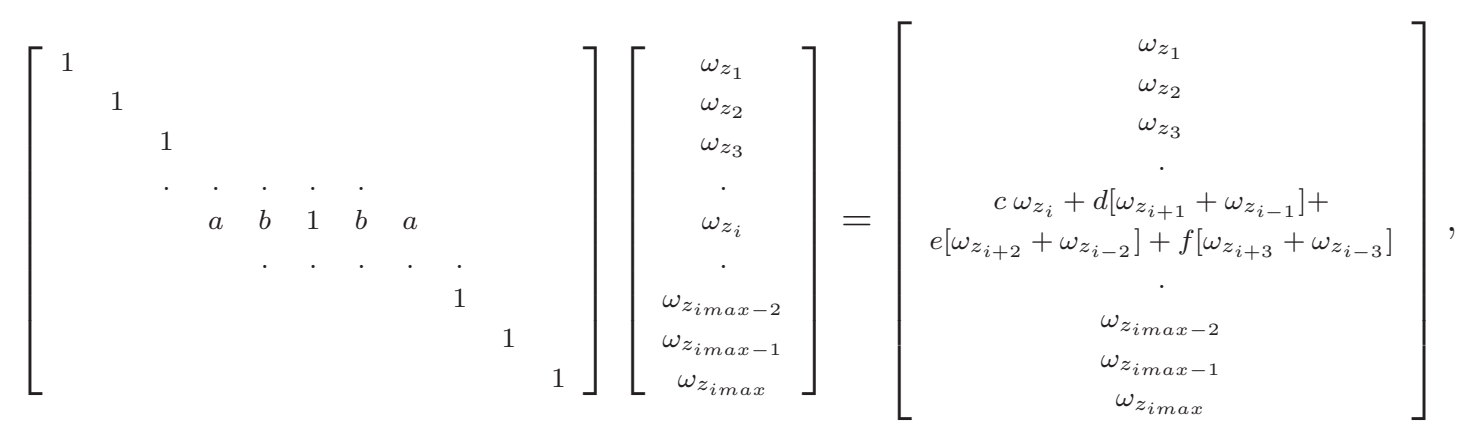

em que $\omega_{z}^{*}$ representa a vorticidade antes da aplicação do filtro computacional. Define-se também as constantes $a=0.1702929, b=0.6522474, c=0.9891856, d=0.66059, e=0.1666774 \mathrm{e}$ $f=0.000679925$.

\subsubsection{O método numérico}

Considerando a formulação vorticidade-velocidade, as equações de transporte de vorticidade, equação de Poisson e a equação da continuidade, definidas respectivamente pela equações (2.11), (2.12) e (2.13), são resolvidas numericamente pelos seguintes passos:

- aplicar um subpasso do integrador temporal;

- aplicar as funções responsáveis pelo amortecimento e relaminarização do escoamento;

- introduzir, a partir de um tempo $t>0$, perturbações próximas a entrada do domínio através de injeção e sucção nas paredes;

- calcular o lado direito da equação de Poisson, dada pela equação (2.12);

- calcular o valor de $v$, por meio da resolução do sistema linear decorrente da equação (2.12);

- calcular o valor de $u$, pela equação da continuidade, representada por (2.13);

- atualizar o valor da vorticidade $\omega_{z}$ nas paredes.

Após o último subpasso do integrador temporal, aplica-se o filtro computacional. O método numérico é finalizado quando atinge-se o tempo limite de simulação.

O próximo capítulo volta-se para a descrição de questões de paralelização do método numérico utilizado. 


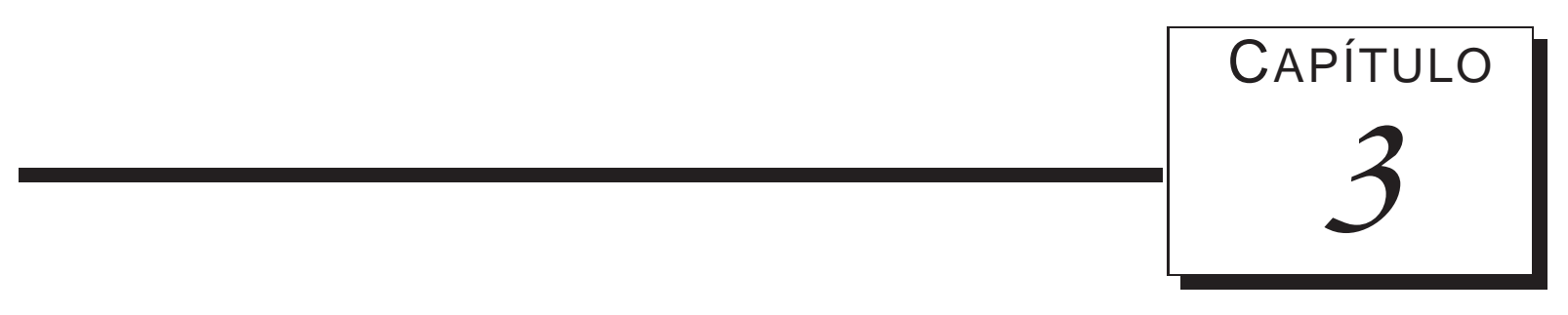

\section{Técnicas de paralelização}

Descreve-se, no presente capítulo, questões associadas à paralelização do método numérico utilizado. São apresentadas estratégias voltadas à resolução de métodos multigrid, paralelização de códigos referentes à geração de aproximações compactas de alta ordem e aplicação do filtro computacional.

\subsection{Paralelização de métodos multigrid}

No que se refere à paralelização dos métodos multigrid apresentados, propõe-se a divisão do domínio do problema apenas na direção $x$. Essa escolha é justificada devido ao fato do número de pontos utilizados na direção $x$ ser significativamente maior que os utilizados na direção $y$, nos problemas de mecânica dos fluidos que se deseja resolver.

Neste sentido, considerando o nível referente à malha mais refinada e um domínio inicial contendo imax pontos na direção $x$ e jmax pontos na direção $y$, cada elemento de processamento é responsável por um subdomínio contendo $N x$ pontos na direção $x$ e jmax pontos na direção $y$. O valor associado à $N x$ é calculado por meio de:

$$
N x=\frac{i \max +(\text { inter }+1)(p-1)}{p},
$$

sendo $p$ o número de elementos de processamento utilizados e inter a sobreposição associada à malha mais refinada com espaçamento entre pontos $(h)$. Torna-se interessante ressaltar que o tamanho da molécula computacional define o número de informações (colunas) a serem transmitidas entre elementos de processamento. 
A sobreposição entre os subdomínios é obtida por meio da relação abaixo:

$$
\text { inter }=2^{(N-1)}(m-2),
$$

sendo $N$ o número de níveis utilizados e $m$ o comprimento da molécula computacional. Por exemplo, se for considerada a aproximação

$$
\frac{\partial^{2} v}{\partial x^{2}}=\frac{v_{i+1}-2 v_{i}+v_{i-1}}{\Delta x^{2}}+O\left(\Delta x^{2}\right)
$$

o comprimento da molécula computacional é $m=3$. Além disso, a utilização dessas expressões pode ser explicitada considerando um exemplo, ilustrado pela figura 3.1. Para esse caso considera-se um método multigrid composto por quatro níveis, ou seja, $N=4$. Neste sentido, tem-se que $i n t e r=$ 8 células. Nota-se que a informação associada a apenas uma coluna é transmitida, tomando o cuidado de sempre manter a concordância com o nível menos refinado $(8 h)$.

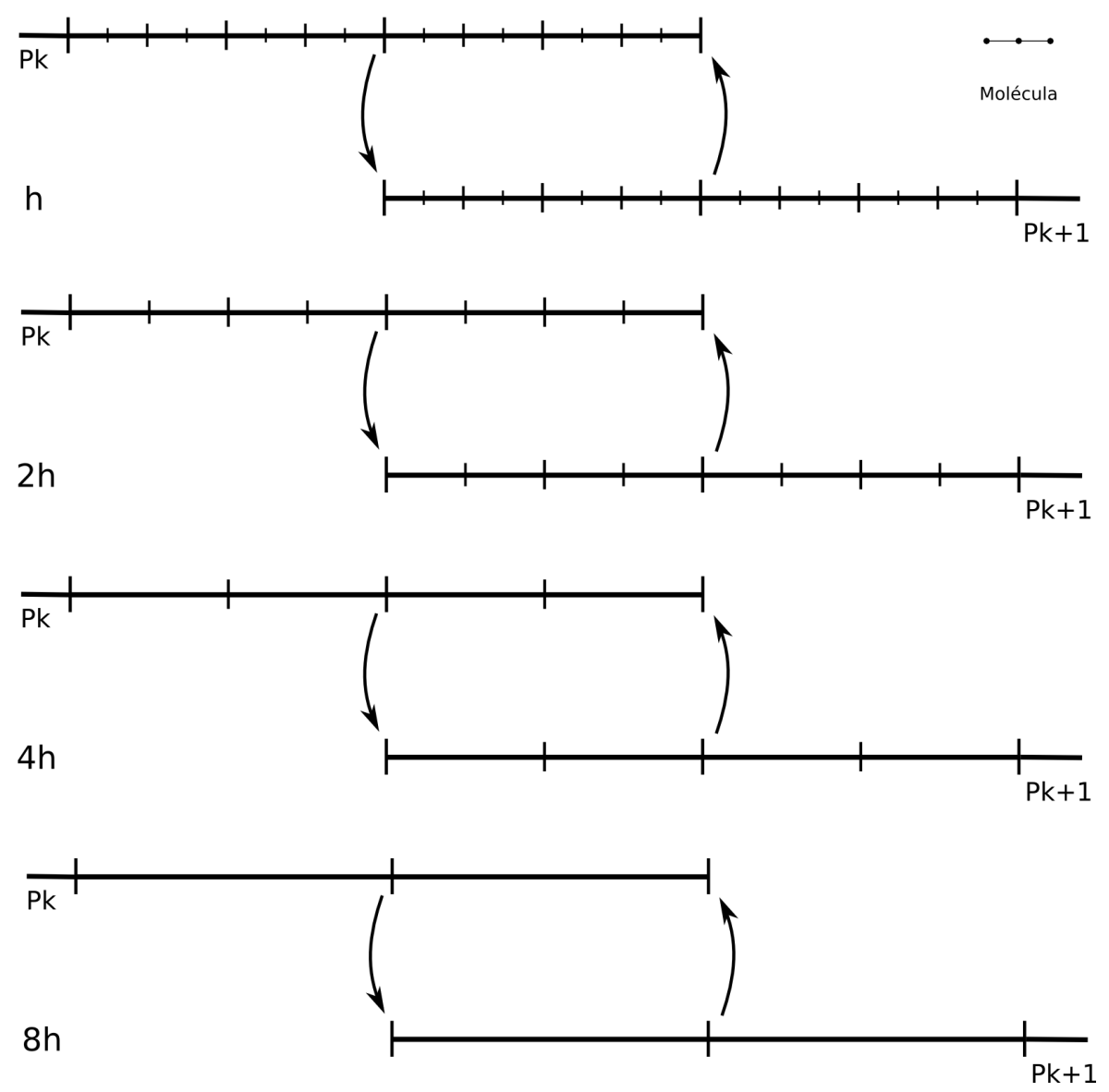

Figura 3.1: Intersecção entre domínios

Vale observar também que, em cada subdomínio, há a aplicação dos métodos anteriormente citados com o acréscimo de pontos de comunicação entre subdomínios adjacentes. No caso do método multigrid CS, essa comunicação dá-se: $(a)$ antes da aplicação da operação de restrição; $(b)$ antes de 
cada passo associado a aplicação do suavizador e $(c)$ antes da interpolação. No que se refere a aplicação do método FAS, a comunicação é realizada: $(a)$ entre a correção e a interpolação; $(b)$ depois da aplicação da operação de restrição; $(c)$ antes de cada iteração do suavizador. Em ambos os casos, a informação a ser transmitida refere-se ao valor da variável $v$.

Os retângulos presentes nas figuras 3.2(a) e 3.2(b) ilustram os pontos de comunicação utilizados, nos métodos CS e FAS, respectivamente.

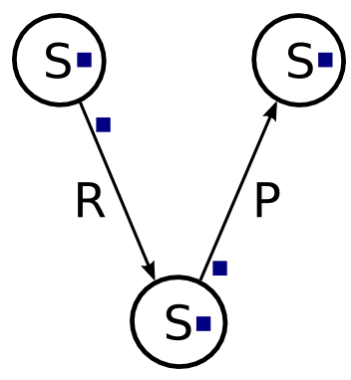

(a) esquema CS

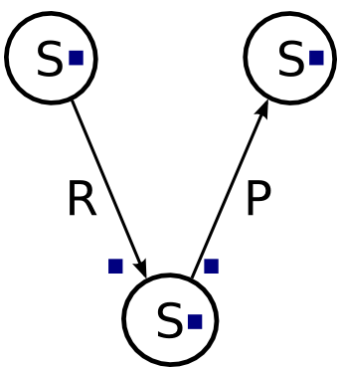

(b) esquema FAS

Figura 3.2: Pontos de comunicação: 3.2(a) esquema CS; 3.2(b) esquema FAS.

O critério de parada utilizado toma como referência o valor máximo absoluto do resíduo em cada subdomínio em questão. O valor obtido é comparado com uma tolerância pré-estabelecida.

\subsection{Diferenças compactas de alta ordem paralelizadas}

Tomando como referência o algoritmo de Thomas, são criadas duas estratégias visando a paralelização do cálculo de derivadas utilizando diferenças compactas. A estratégia de paralelização I emprega o conceito de pipeline enquanto a estratégia II tira proveito da intersecção decorrente do uso da decomposição de domínio necessária para os esquemas multigrid. Os métodos citados são descritos na sequência.

De maneira geral, considera-se um domínio discreto de dimensão imax $\times$ jmax. Define-se como $p$ o número de elementos de processamento envolvidos. Toma-se ainda $N x$ como a dimensão em $x$ de cada subdomínio do problema e inter como a constante relacionada à intersecção entre subdomínios adjacentes.

\subsubsection{Algoritmo sequencial - Algoritmo clássico de Thomas}

O Algoritmo de Thomas, também conhecido como tridiagonal matrix algorithm (TDMA) é uma adaptação do método da eliminação de Gauss voltada à resolução de sistemas de equações na forma tridiagonal.

Considerando um sistema linear tridiagonal dado por: 


$$
\left[\begin{array}{ccclccc}
b_{1} & c_{1} & 0 & \ldots & & & \\
a_{2} & b_{2} & c_{2} & \ldots & & & \\
& & & \ldots & & & \\
& & & \ldots & a_{i \max -1} & b_{i \max -1} & c_{i \max -1} \\
& & & \ldots & 0 & a_{\text {imax }} & b_{\text {imax }}
\end{array}\right]\left[\begin{array}{c}
u_{1} \\
u_{2} \\
\vdots \\
u_{i \max -1} \\
u_{\text {imax }}
\end{array}\right]=\left[\begin{array}{c}
d_{1} \\
d_{2} \\
\vdots \\
d_{i \max -1} \\
d_{\text {imax }}
\end{array}\right]
$$

A primeira etapa do algoritmo consiste em calcular:

$$
\begin{aligned}
c_{i}^{\prime}=\left\{\begin{aligned}
\frac{c_{i}}{d_{i}}, & \text { para } i=1, \\
\frac{c_{i}}{b_{i}-c_{i-1}^{\prime} a_{i}}, & \text { para } i=2, \ldots, \text { imax },
\end{aligned}\right. \\
d_{i}^{\prime}=\left\{\begin{aligned}
\frac{d_{i}}{b_{i}}, & \text { para } i=1, \\
\frac{d_{i}-d_{i-1}^{\prime} a_{i}}{b_{i}-c_{i-1}^{\prime} a_{i}}, & \text { para } i=2, \ldots, i \max .
\end{aligned}\right.
\end{aligned}
$$

A segunda etapa consiste em calcular:

$$
\left\{\begin{array}{l}
u_{i \max }=d_{i \max }^{\prime}, \\
u_{i}=d_{i}^{\prime}-c_{i}^{\prime} u_{i+1}, \quad \text { para } i=i \max -1, i \max -2, \ldots, 1 .
\end{array}\right.
$$

Na sequência, são apresentadas duas técnicas computacionais com o objetivo de paralelizar a solução de sistemas tridiagonais. Ambos os métodos implementados baseiam-se no algoritmo clássico de Thomas.

\subsubsection{Estratégia de paralelização I}

O problema de se estimar as derivadas no domínio em questão pode ser observado da seguinte forma:

A primeira etapa volta-se para a eliminação dos elementos abaixo da diagonal principal e a resolução do sistema triangular inferior (associado a matriz $L$ da decomposição $L U$ ) por substituição direta, para todas as linhas do domínio do problema. A segunda etapa objetiva o cálculo de todas as matrizes triangulares superiores $U$ e, como consequência, o término da resolução dos sistemas tridiagonais em questão.

A paralelização, tomando como referência as duas etapas anteriormente apresentadas, consiste em utilizar o conceito de pipeline. O esquema apresentado pela tabela 3.1 ilustra a estratégia de pipeline voltada à primeira etapa da resolução dos sistemas tridiagonais. A resolução da segunda etapa é realizada de forma similar.

Por facilidade, o domínio é decomposto da mesma forma que a realizada para a aplicação do método multigrid. Para tanto, considera-se o uso de $p$ elementos de processamento, representados 
pelas colunas da tabela 3.1 e denotados por $P_{k}, k=1,2, \ldots, p$. Toma-se ainda as linhas da tabela 3.1 como estágios do pipeline. A matriz triangular inferior é divida em $p$ partes, de acordo com a estrutura utilizada pelo método multigrid. Cada parte é denotada por $L_{k}, k=1, \ldots, p$.

Além disso, considerando que objetiva-se a aproximação das derivadas em um domínio retangular, $j_{p, q}$ denota a parte do sistema tridiagonal referente ao subdomínio $q$ e a linha $p$ do domínio retangular. As posições marcadas com o símbolo (-) representam a não utilização do recurso devido ao término de processamento ou à espera de informações provenientes do elemento de processamento anterior.

Tabela 3.1: Estratégia de paralelização I - Aproximação de derivadas por diferenças compactas de alta ordem utilizando o conceito de pipeline.

\begin{tabular}{|c|c|c|c|c|c|}
\hline estágio/elemento de proc. & $P_{1}$ & $P_{2}$ & $P_{3}$ & $\ldots$ & 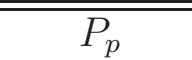 \\
\hline 1 & $j_{1,1} L_{1}$ & - & - & - & - \\
\hline 2 & $j_{2,1} L_{1}$ & $j_{1,2} L_{2}$ & - & - & - \\
\hline 3 & $j_{3,1} L_{1}$ & $j_{2,2} L_{2}$ & $j_{1,3} L_{3}$ & - & - \\
\hline$\vdots$ & $\vdots$ & $\vdots$ & $\vdots$ & - & - \\
\hline$p$ & $j_{p, 1} L_{1}$ & $j_{p-1,2} L_{2}$ & $j_{p-2,3} L_{3}$ & ․ & $j_{1, p} L_{p}$ \\
\hline$\vdots$ & $\vdots$ & $\vdots$ & $\vdots$ & & $\vdots$ \\
\hline$n$ & $j_{n, 1} L_{1}$ & $j_{n-1,2} L_{2}$ & $j_{n-2,3} L_{3}$ & . & $j_{n-p, p} L_{p}$ \\
\hline$n+1$ & - & $j_{n, 2} L_{2}$ & $j_{n-1,3} L_{3}$ & $\cdots$ & $j_{n-p+1, p} L_{p}$ \\
\hline$n+2$ & - & - & $j_{n, 3} L_{3}$ & .. & $j_{n-p+2, p} L_{p}$ \\
\hline$\vdots$ & - & - & - & .. & $\vdots$ \\
\hline$n+p$ & - & - & - & - & $j_{n, p} L_{p}$ \\
\hline
\end{tabular}

De forma geral, a paralelização da primeira etapa, para uma dada linha, dá-se da seguinte forma:

- o elemento de processamento $k=1$ efetua as operações referentes à geração da matriz $L$, da 
decomposição $L U$, do ponto 1 até o ponto $N x-2$;

- o elemento de processamento $k, k>1$ aguarda o recebimento de informações associadas às inter últimas linhas do subdomínio $k-1$ e os associam às primeiras linhas do subdomínio $k$;

- o elemento de processamento $k$, para $1<k<p$, efetua as operações associadas ao cálculo da matriz $L$ partindo-se do ponto inter -1 até o ponto $N x-2$. O elemento de processamento $k=p$ realiza a decomposição até o ponto $N x$;

- o elemento de processamento $k \operatorname{com} k<p$, transmite informações associadas às inter últimas incógnitas para o elemento de processamento $k+1$.

\subsubsection{Estratégia de paralelização II}

A figura 3.3(a) ilustra o comportamento do erro absoluto cometido pela aplicação do algoritmo sequencial de Thomas no sentido de aproximar a derivada segunda da função $v(x, y)=\operatorname{sen}(x)$. Para esse experimento, toma-se o espaçamento como $\Delta x=0.2 \mathrm{em}$ um domínio que varia de 0 à 57.6. Nota-se que a redução na ordem das aproximações próximas aos contornos, e o fato das mesmas serem descentradas, implica erros significativamente maiores.

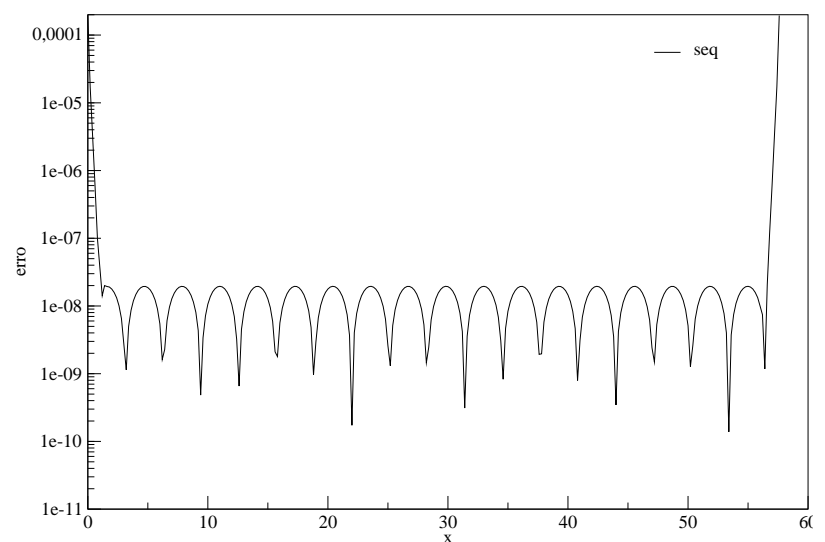

(a) caso sequencial

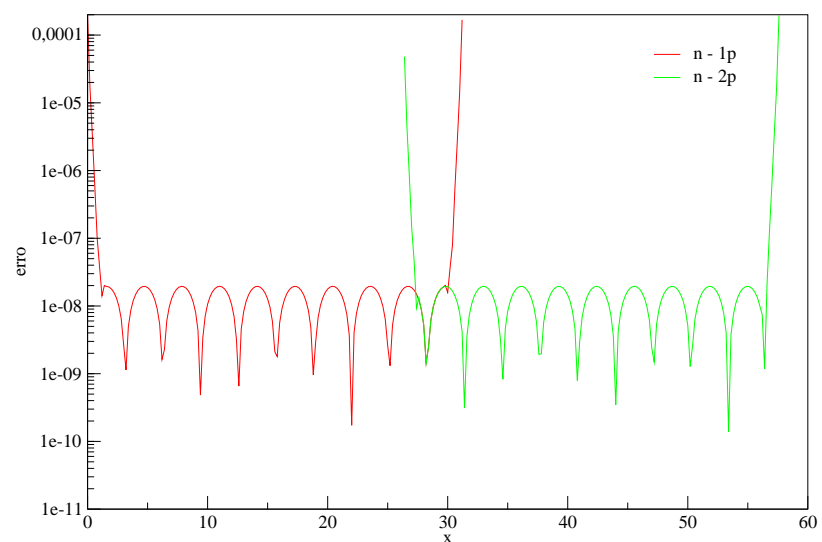

(b) decomposição de domínio

Figura 3.3: Comportamento do erro: 3.3(a) caso sequencial; 3.3(b) decomposição de domínio.

Uma alternativa direta de paralelização refere-se à utilização da estratégia de decomposição de domínio, já explicitada em meio às considerações referentes ao método multigrid. Neste sentido, é aceitável supor que cada subdomínio (ou elemento de processamento) fique responsável por gerar aproximações para a derivada em questão de forma independente em relação aos demais. Ressalta-se que a presente estratégia não se volta para a paralelização da resolução de um sistema tridiagonal.

O erro cometido por meio dessa estratégia de paralelização é apresentado na figura 3.3(b). Por meio dela, verifica-se que a independência entre os domínios gera um inconveniente. Na região de intersecção entre os domínios, tomando como referência uma mesma coordenada, há uma significativa 
diferença entre o erro cometido por cada elemento de processamento. Uma forma de contornar esse problema consiste em, ao término do cálculo de todas as aproximações, trocar informações entre elementos de processamento adjacentes na tentativa de sempre considerar a melhor aproximação.

Experimentalmente, foi possível identificar que a aproximação compacta de ordem reduzida utilizada no contorno afeta, de forma significativa, apenas os inter $/ 2$ pontos próximos aos extremos, quando inter $=24$. Neste sentido, propõe-se a seguinte estratégia de comunicação: após o término do cálculo de todos os sistemas, o elemento de processamento $k$ envia ao elemento de processamento $k+1$, as colunas $N x$-inter até $N x$-inter $/ 2$. Além disso, o elemento de processamento $k$ aguarda o recebimento de inter $/ 2$ colunas referentes ao elemento de processamento $k+1$ e os armazena nas últimas inter $/ 2$ colunas. O esquema de comunicação é ilustrado pela figura 3.4.

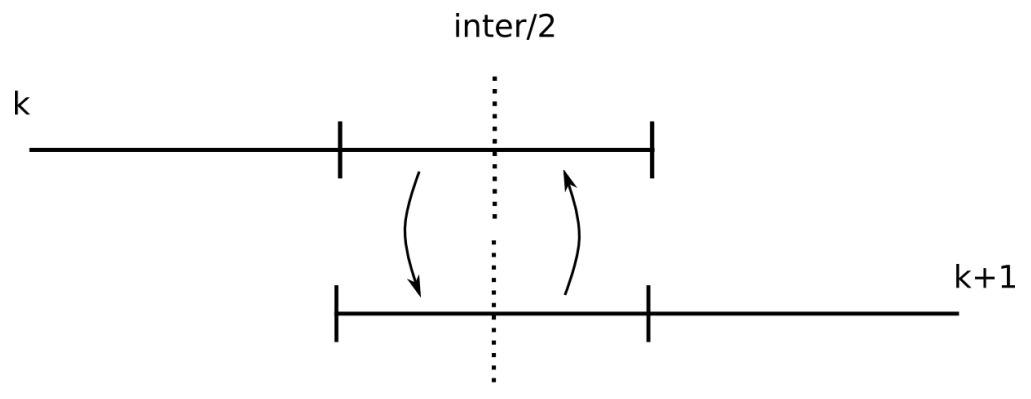

Figura 3.4: Estratégia de paralelização II - Comunicação entre elementos de processamentos.

\subsection{A paralelização na filtragem espacial}

A resolução sequencial do sistema pentadiagonal em questão dá-se pela decomposição da matriz A no produto LU, sem pivotamento. A solução final também é obtida por meio da resolução dos sistemas triangulares decorrentes da decomposição.

\subsubsection{Filtragem espacial - Estratégia de paralelização I}

A abordagem utilizada é similar a estratégia de paralelização I para aproximação de derivadas. A matriz $A$, de dimensão $n \times 5$ é alocada em cada elemento de processamento $k$ associado à decomposição do domínio. Cada elemento de processamento realiza a mesma decomposição $L U$ da matriz $A$, de forma independente.

A paralelização, utilizando o conceito de pipeline, explora a resolução dos sistemas triangulares. Cada elemento de processamento fica responsável pelo cálculo de $N x-3$ incógnitas do sistema triangular. Exceções são observadas no primeiro e último subdomínio, para o caso triangular inferior e superior, respectivamente. Para esses casos, todo o sistema deve ser resolvido.

De forma geral, considera-se um laço em $j$, variando de 2 à $j$ max - 1. Para um dado $j$ fixo, cada elemento de processamento $k$, exceto o primeiro:

- aguarda o recebimento das três últimas incógnitas do elemento de processamento $k-1$ e as atribui as três primeiras incógnitas em $k$; 
- realiza parte da resolução do sistema triangular inferior;

- envia as últimas três incógnitas para o elemento de processamento $k+1$.

Após finalizado o laço referente ao cálculo da solução do sistema triangular inferior, inicia-se a resolução do sistema triangular superior, e como consequência, a obtenção da solução do sistema pentadiagonal. Na segunda etapa da paralelização, o mesmo laço em $j$ deve ser considerado. No entanto, para um $j$ fixo, cada elemento de processamento $k$, exceto o último:

- aguarda o recebimento das três primeiras incógnitas do elemento de processamento $k+1$ e as atribui as três últimas incógnitas em $k$;

- realiza parte da resolução do sistema triangular superior;

- envia as primeiras três incógnitas para o elemento de processamento $k-1$.

\subsubsection{Filtragem espacial - Estratégia de paralelização II}

De forma análoga ao caso apresentado para o sistema tridiagonal, a estratégia utilizada pelo método consiste em dividir o domínio em $p$ subdomínios e considerá-los independentes entre si. Ao término do cálculo da solução em cada subdomínio, troca-se informações entre domínios adjacentes na tentativa de minimizar o erro.

O capítulo a ser apresentado objetiva a descrição e análise dos principais resultados obtidos por meio do presente projeto de pesquisa. 


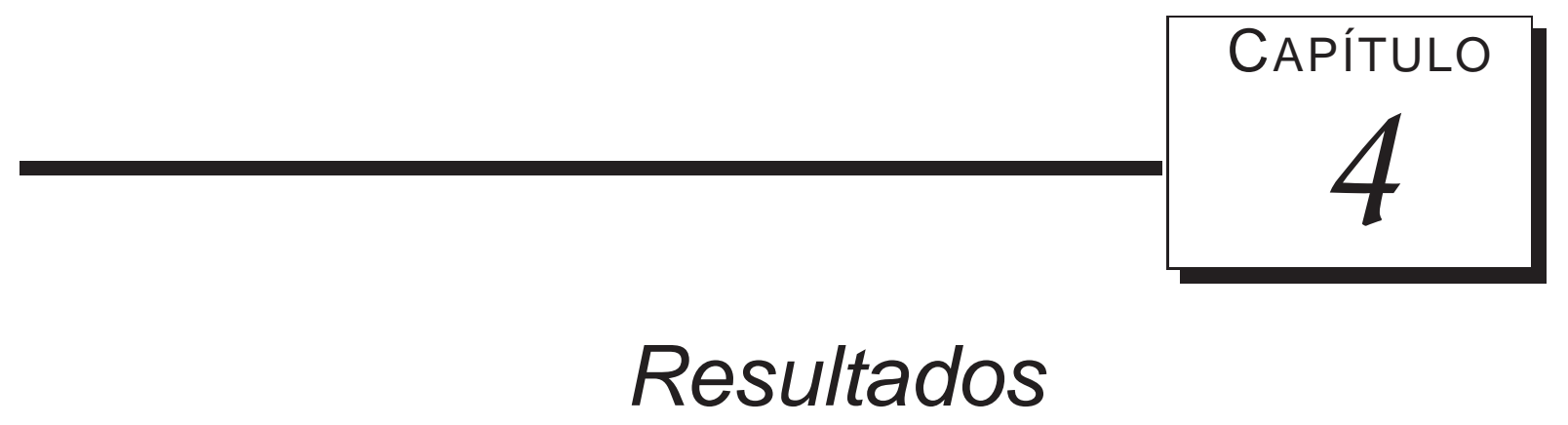

Apresenta-se, neste capítulo, os principais resultados obtidos. São contemplados tópicos referentes às vantagens na utilização de métodos multigrid, forma de alocação de estrutura de dados, comparativo entre estratégias de paralelização de métodos multigrid e técnicas de aproximação compacta de alta ordem. Investiga-se também como essas técnicas de paralelização influenciam nos resultados referentes à simulação de escoamento de Poiseuille bidimensional. Os resultados mais relevantes dispostos na forma de gráfico, neste capítulo, tem seus valores apresentados na forma de tabelas no Apêndice A, no final desta dissertação.

\subsection{Paralelização de métodos multigrid}

Considera-se a equação de Poisson bidimensional em um domínio retangular. No que se refere às condições de contorno, tem-se dois casos de testes:

- Caso I: condição de contorno de Dirichlet igual à $v(x, y)=\operatorname{sen}(x)\left(y^{5}-y^{4}-y^{3}+y^{2}\right)=$ $\operatorname{sen}(x) q(y)$ em todos os limites do domínio;

- Caso II: condição de contorno do tipo Neumann no contorno superior, e condição de contorno de Dirichlet igual ao caso anterior para os demais contornos.

Esses casos são adotados, pois são necessários para a solução de escoamentos entre paredes (caso I) e camada limite (caso II). O termo fonte para ambos os casos é tomado como $s=\operatorname{sen}(x)\left[q^{\prime \prime}-q\right]$. $\mathrm{O}$ valor de $\Delta y$, definido como sendo a distância entre pontos de malha consecutivos na direção $y$, é computado como sendo $\Delta y=\frac{1}{j m a x-1}$. Toma-se ainda $\Delta x=\Delta y$, como sendo a distância entre pontos de malha consecutivos na direção $x$. O critério de parada leva em consideração o máximo resíduo absoluto, com valor menor que $10^{-5}$. 
São implementadas versões paralelizadas para os métodos multigrid CS e FAS. Adota-se, para ambos os métodos multigrid, um ciclo $\mathrm{V}$ composto por quatro níveis. O método CS empregado utiliza o método de Jacobi por linhas subrelaxado em todos os níveis do ciclo V, exceto no menos refinado, onde utiliza-se o método SOR por linhas. Em relação ao método FAS, o método Gauss-Seidel por linhas é aplicado em situações onde há aplicação de apenas uma iteração. Para os demais casos, o método SOR por linhas é utilizado.

Os fatores de relaxação empregados para o método de Jacobi e SOR são, respectivamente, $r_{1}=$ 0.9 e $r_{2}=1.1$. Considerando o método multigrid CS, emprega-se $N_{2}=100$ iterações para o nível menos refinado e $N_{1}=2$ iterações para os demais. Em relação ao método multigrid FAS, os mesmos parâmetros são utilizados. Adicionalmente, uma iteração é aplicada nos níveis intermediários associados a parte direita do ciclo $\mathrm{V}$, ou seja, com $N_{3}=1$.

Os testes são gerados utilizando 1, 2, 4 e 8 elementos de processamento, utilizando processadores Intel Xeon E5345 com 2.33Ghz, 16Gb de memória RAM, todos com 8 elementos de processamento. Além disso, os resultados referentes a tempo de execução aqui apresentados são obtidos por meio de uma média aritmética gerada por quatro simulações.

A tabela 4.1 explicita o conjunto de malhas utilizado. Faz-se interessante ressaltar que para um bloco onde jmax é constante, não há variação de $\Delta x$, caracterizando um aumento do domínio a ser discretizado.

Tabela 4.1: Eixo nominal - malhas utilizadas - molécula computacional de 5 pontos.

\begin{tabular}{|c|c|c|c|c|c|c|c|c|}
\hline & & & & \\
\hline $\mathrm{N}$ & imax & $\overline{\text { jmax }}$ & $\overline{\mathrm{N}}$ & imax & $\overline{\mathrm{jmax}}$ & $\mathrm{N}$ & imax & $\overline{\mathrm{jmax}}$ \\
\hline 0 & 153 & 33 & 12 & 153 & 129 & 24 & 153 & 513 \\
\hline 1 & 281 & 33 & 13 & 281 & 129 & 25 & 281 & 513 \\
\hline 2 & 537 & 33 & 14 & 537 & 129 & 26 & 537 & 513 \\
\hline 3 & 1049 & 33 & 15 & 1049 & 129 & 27 & 1049 & 513 \\
\hline 4 & 2073 & 33 & 16 & 2073 & 129 & 28 & 2073 & 513 \\
\hline 5 & 4121 & 33 & 17 & 4121 & 129 & 29 & 4121 & 513 \\
\hline 6 & 153 & 65 & 18 & 153 & 257 & 30 & 153 & 1025 \\
\hline 7 & 281 & 65 & 19 & 281 & 257 & 31 & 281 & 1025 \\
\hline 8 & 537 & 65 & 20 & 537 & 257 & 32 & 537 & 1025 \\
\hline 9 & 1049 & 65 & 21 & 1049 & 257 & 33 & 1049 & 1025 \\
\hline 10 & 2073 & 65 & 22 & 2073 & 257 & 34 & 2073 & 1025 \\
\hline 11 & 4121 & 65 & 23 & 4121 & 257 & 35 & 4121 & 1025 \\
\hline
\end{tabular}

\subsubsection{Esquemas multigrid versus singlegrid}

Com o intuito de explicitar a diminuição do tempo de execução de métodos multigrid em relação a métodos singlegrid, apresenta-se um comparativo entre o método multigrid CS e o método singlegrid SOR por linhas, com fator de relaxação $r=1.1$. Os parâmetros utilizados para o método multigrid foram descritos na seção anterior. 
Esse comparativo volta-se para a resolução de uma equação de Poisson em um domínio retangular, sujeita as condições dadas pelo caso I. O critério de parada leva em consideração o resíduo máximo absoluto na malha mais refinada, que deve ser menor do que $10^{-5}$. O conjunto de malhas utilizado é descrito pela tabela 4.1 .

A figura 4.1 ilustra uma razão de melhoramento, gerada por meio da seguinte expressão:

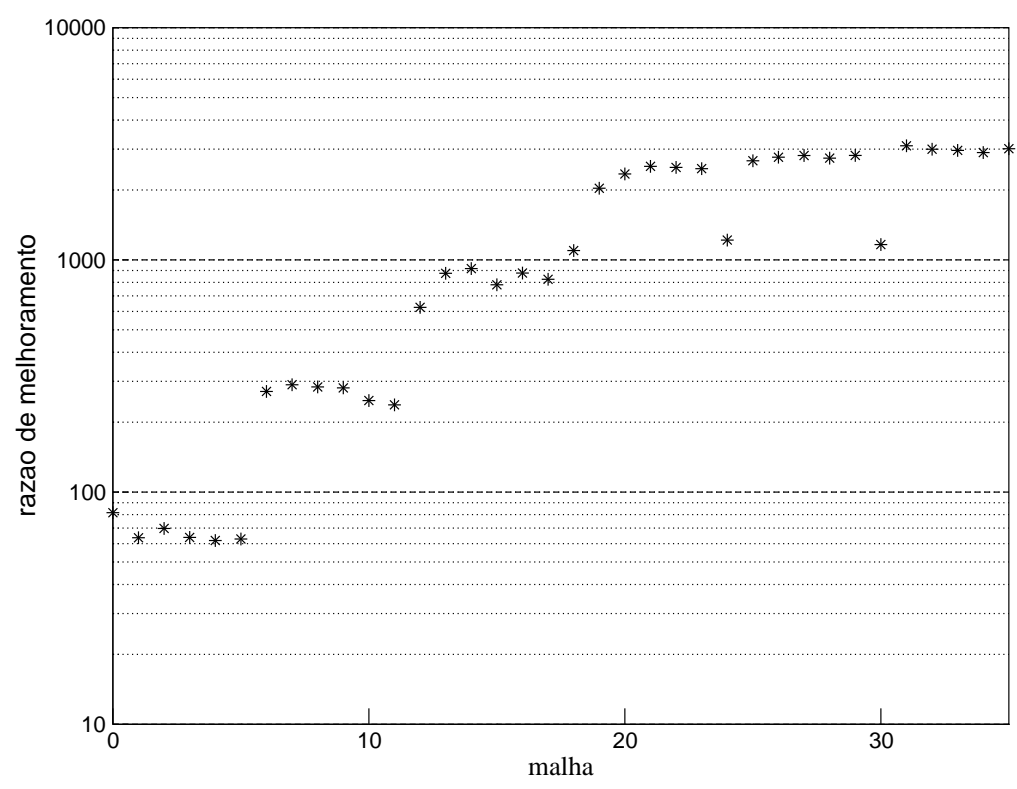

Figura 4.1: Razão de melhoramento entre os métodos SOR sequencial e o método multigrid CS sequencial.

$$
\text { razão de melhoramento }=\frac{\text { tempo de execução do método singlegrid }}{\text { tempo de execução do método multigrid }} .
$$

Nota-se claramente, por meio da figura 4.1, ganhos significativos de tempo, principalmente com o aumento do número de variáveis utilizadas.

\subsubsection{Influência da alocação da estrutura de dados}

Diversas são as variáveis que influenciam, direta ou indiretamente, no desempenho de um código computacional. Cita-se como variáveis: recursos físicos, sistema operacional, compilador (e respectivas tags de otimização) e linguagem de programação adotada.

Utiliza-se, para o presente trabalho, a linguagem de programação FORTRAN. Além disso, a representação e armazenamento das informações contidas nos diversos níveis do método multigrid dá-se por meio de uma estrutura de dados de dimensão 3. Justifica-se essa escolha pela simplicidade no acesso a um determinado nível e pela generalização que a estrutura tridimensional proporciona. Sendo assim, considerando um código multigrid sequencial, a estrutura $v$ (imax, jmax, msh) é capaz de armazenar a solução da equação de Poisson em todos os msh níveis do método multigrid. Neste 


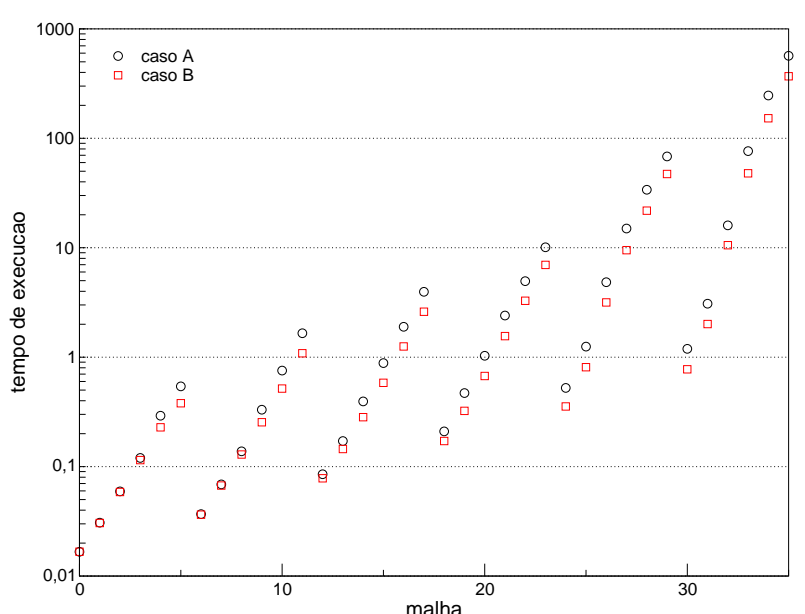

(a)

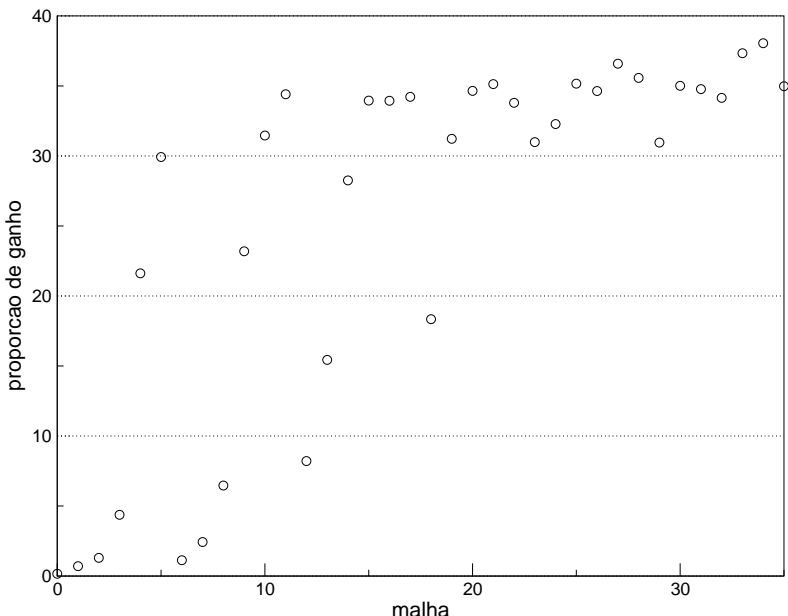

(b)

Figura 4.2: Influência da alocação de estrutura de dados utilizando o método multigrid FAS.

sentido, considerando o método multigrid FAS sequencial, para o caso I, realizou-se uma investigação no sentido de mensurar o impacto que a forma de alocação desta estrutura de dados causa frente ao tempo de execução. Assim, dois casos são considerados:

- caso A: A estrutura de dados é alocada na forma $v($ msh, imax, jmax);

- caso B: A estrutura de dados é alocada na forma $v($ imax, jmax, msh).

O gráfico representado pela figura 4.2(a) apresenta o tempo de execução do código multigrid FAS sequencial, considerando os casos $\mathrm{A}$ e $\mathrm{B}$, anteriormente propostos. No eixo $x$, são representados nominalmente - os conjuntos de pontos utilizados, em concordância com a tabela 4.1. O tempo de execução, em segundos, é representado pelo eixo y, em escala logarítmica e obtido pela média de 4 simulações. Além disso, a figura 4.2(b) apresenta a proporção de melhoramento ou diferença entre as duas estratégias. O eixo y representa o ganho percentual obtido com a utilização do caso B. O cálculo do percentual de ganho é gerado por meio da expressão:

$$
\% \text { de ganho }=\frac{t e_{A}-t e_{B}}{t e_{A}} \cdot 100
$$

sendo $t e_{A}$ o tempo de execução do código utilizando o caso A e te $e_{B}$ o tempo de execução do código utilizando o caso $\mathrm{B}$, em segundos.

Considerando o tempo de execução dos códigos em questão, a diferença entre as estratégias A e B é relativamente pequena, para malhas com um pequeno número de pontos. A afirmação pode ser explicitada para o método multigrid em questão, considerando as malhas $0,1,2,3,6,7,8,12,13$ e 18 onde há ganhos inferiores a $20 \%$. No entanto, há diminuição do tempo de execução para todos os casos testado e, de forma geral, ganhos de até $40 \%$ podem ser observados. 


\section{1 - Paralelização de métodos multigrid}

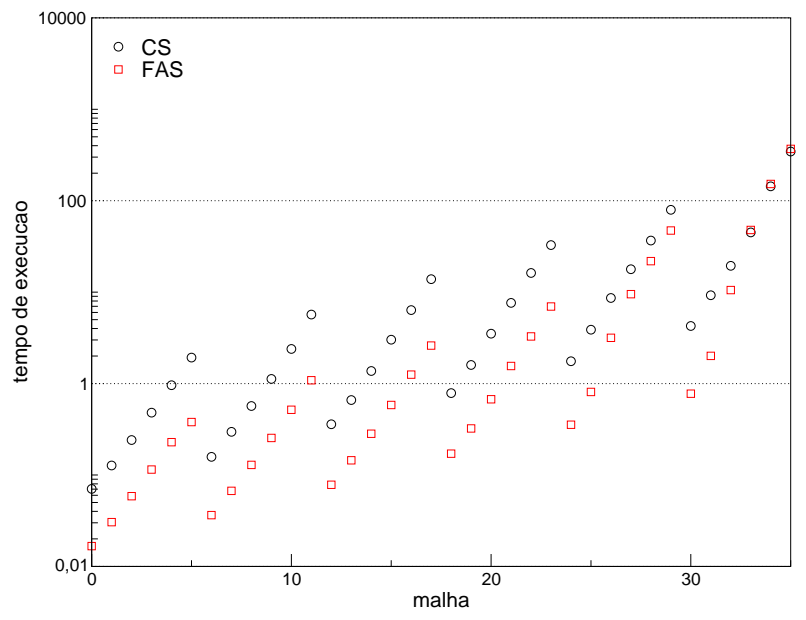

(a) caso I

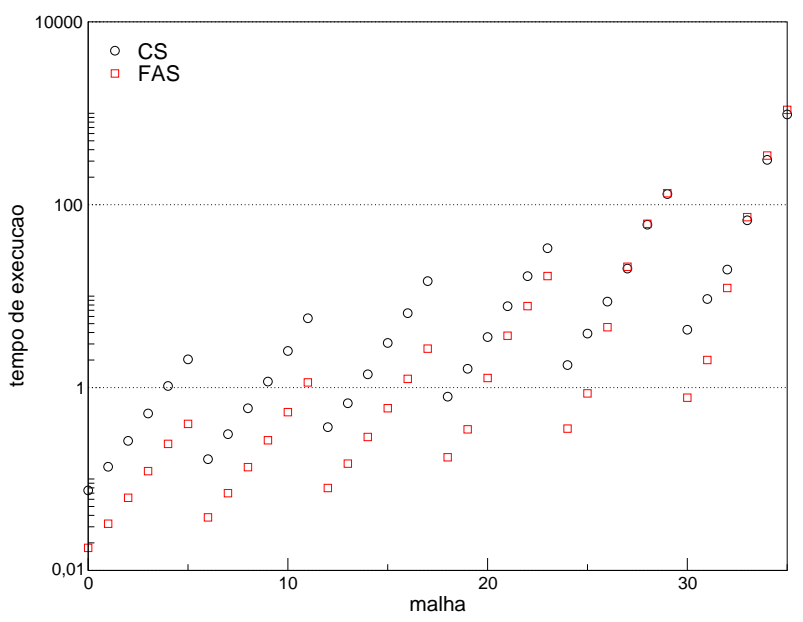

(b) caso II

Figura 4.3: Tempo de execução dos métodos multigrid CS e FAS sequenciais.

\subsubsection{Comparação de métodos multigrid CS e FAS}

Os gráficos 4.3(a) e 4.3(b) representam o tempo de execução de ambos os métodos multigrid sequenciais, para os casos $I$ e $I I$, respectivamente. O eixo $x$, em concordância com a tabela 4.1, representa o conjunto de malhas utilizado. O tempo de execução, em segundos, é representado pelo eixo $y$, em escala logarítmica.

Considerando a figura 4.3(a), o método multigrid FAS utiliza tempos de execução inferiores se comparados com o método CS. O fato é explicitado devido a significativa diminuição do número de ciclos V que o método FAS demanda em relação ao método CS. Para um conjunto de malhas cujo Ny é fixo, percebe-se um crescimento exponencial do tempo de execução gasto por ambos os métodos multigrid testados. Nota-se também que a variação do tempo de execução dos métodos multigrid testados, para grande parte das malhas utilizadas apresenta um certo padrão. No entanto, o aumento do número de pontos implica em uma tendência à diminuição dessa diferença, tornando o método CS mais competitivo. O método CS apresenta índices competitivos para os casos 33, 34 e 35.

Em concordância com os dados apresentados para o caso I, a aplicação do método FAS para o caso II, apresenta os melhores resultados para a maioria dos casos observados. No entanto, a diferença existente entre os tempos de execução dos métodos FAS e CS, para o caso II, é significativamente menor que a diferença apresentada pelo métodos no caso I. Uma menor diferença entre as estratégias testadas fica evidenciada nas malhas 27, 28, 29, 33, 34 e 35, onde o método CS apresenta desempenho igual ou superior ao método FAS. Vale ressaltar ainda que o tempo de execução gasto por ambos os métodos, considerando o caso II, é similar ao tempo de execução empregado no caso I.

Analisando a performance de métodos paralelizados, as figuras 4.4(a) e 4.4(b) representam o speedup referente ao caso I, para os métodos multigrid CS e FAS, respectivamente. Já as figuras 4.6(a) e 4.6(b), analogamente, representam o speedup para os métodos CS e FAS considerando o caso II. De maneira complementar, as figuras 4.5(a) e 4.5(b) representam a eficiência dos métodos 
CS e FAS, considerando o primeiro caso. A eficiência associada ao segundo caso, considerando respectivamente os métodos multigrid CS e FAS, é representada pelas figuras 4.7(a) e 4.7(b).

Define-se speedup como:

$$
S=\frac{T_{1}}{T_{p}}
$$

em que $T_{1}$ representa o tempo de execução de um código com o menor número de elementos de processamento (para o presente caso, considera-se um código paralelizado utilizando apenas um elemento de processamento) e $T_{p}$ o tempo de execução de um código paralelizado. Essa métrica possibilita analisar o quanto um código paralelizado é mais rápido (ou não) que sua versão sequencial. Além disso, define-se a métrica eficiência como:

$$
E=\frac{S}{p}
$$

em que $S$ representa o speedup e $p$ o número de elementos de processamento.

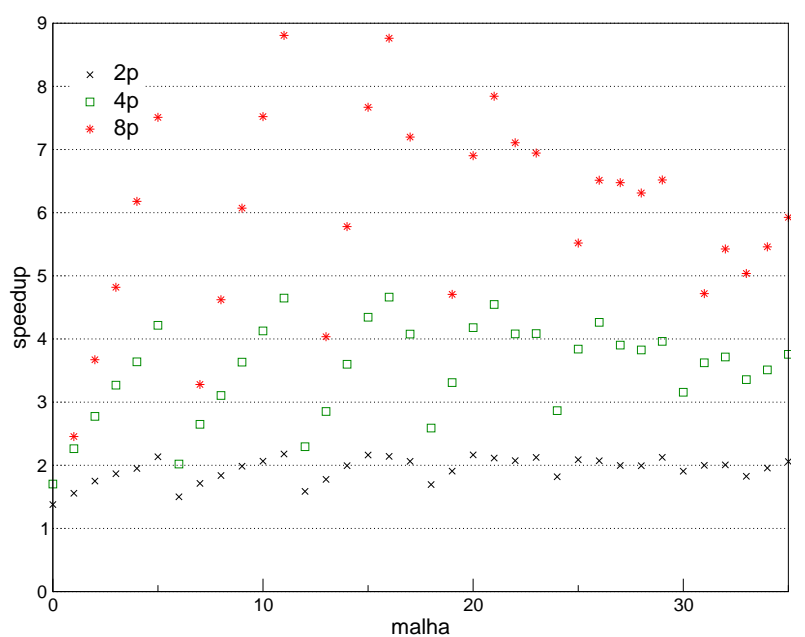

(a) método CS

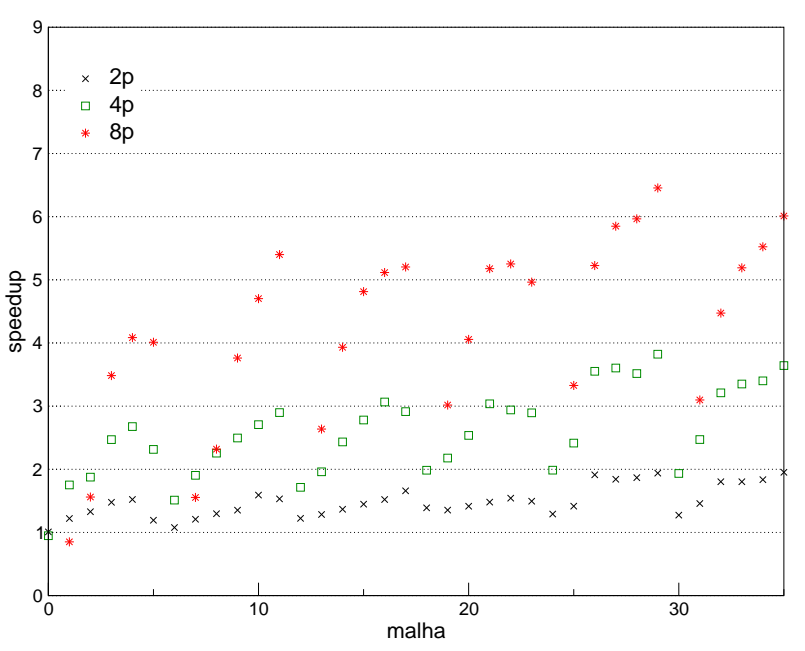

(b) método FAS

Figura 4.4: Speedup dos métodos multigrid paralelizados para o caso I.

Por meio da figura 4.4(a), é possível perceber que ótimos resultados são obtidos pelo uso do método multigrid CS paralelizado, considerando o caso I. Um número significativo de malhas apresentou speedup acima do esperado. Citam-se por exemplo, as malhas 11 e 16 para 4 e 8 elementos de processamento e as malhas $5,10,11,15,16,17,20,21,22,23$ e 26 com 4 elementos de processamento. O código, utilizando 2 elementos de processamento, mostra resultados muito positivos. No entanto, é possível que os índices de speedup, para malhas menos refinadas sofram, de alguma forma, influência de variáveis externas, já que o tempo de execução para esses casos é relativamente pequeno. As maiores variações dos valores obtidos são observadas com a utilização de 8 elementos de processamento. Essa variação pode ser justificada principalmente devido à comunicação entre domínios adjacentes. 
Em relação a figura 4.4(b), observa-se que os maiores índices considerando 4 e 8 elementos de processamento, fixando-se o número de elementos na direção $y$, ocorrem geralmente em malhas com um grande número de pontos na direção $x$, como 2073 e 4121. O aumento do número de pontos nas direções $x$ implica, na maioria dos casos, um aumento nos índices de speedup. O mesmo comportamento pode ser observado em casos onde há aumento do número de pontos na direção $y$.

De forma geral, os índices de speedup para o caso I, considerando a aplicação do método multigrid FAS, não alcançam valores tão expressivos se comparados com o método multigrid CS. Esse fato é justificado pela aplicação de uma iteração do método de Gauss-Seidel na volta do ciclo V referente ao método FAS, a qual implica pontos adicionais de comunicação. O tempo de comunicação referente a esses pontos de comunicação faz com que os índices de speedup caiam. No entanto, considera-se o método FAS competitivo, no sentido que a maioria dos casos testados apresentam bons índices de speedup.

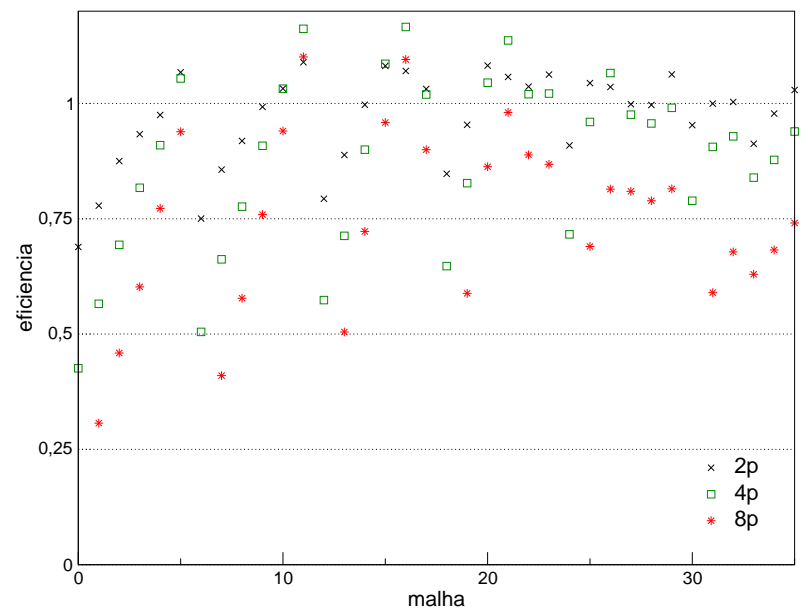

(a) método CS

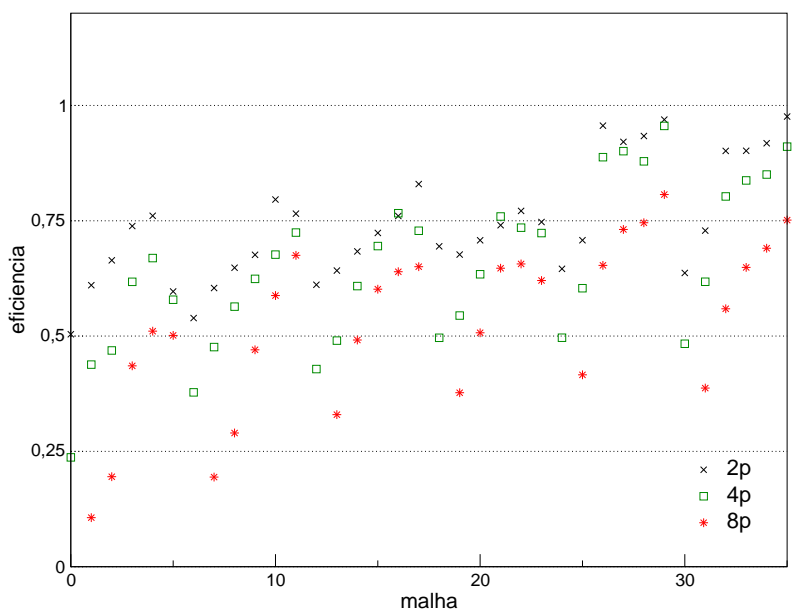

(b) método FAS

Figura 4.5: Eficiência dos métodos multigrid paralelizados para o caso I.

Fica evidenciado, por meio do gráfico 4.5(a), ganhos de tempo acima do esperado para todas as malhas em que o índice de eficiência é maior do que um. Nota-se ainda, por meio das figuras 4.4(a) e 4.5(a) que o método CS apresenta ótimos resultados para todas malhas testadas. Análises voltadas aos gráficos 4.4(b) e 4.5(b) possibilitam afirmar que o método FAS paralelizado é ineficiente apenas nas duas primeiras malhas testadas, considerando respectivamente 4 e 8 elementos de processamento. É possível perceber ainda, por meio dos gráficos 4.5(a) e 4.5(b), os efeitos de comunicação frente ao aumento do número de elementos de processamento. Neste sentido, os menores índices de eficiência geralmente estão associados à 8 elementos de processamento.

Em relação à figura 4.6(a), os índices de speedup para 2 e 4 elementos de processamento apresentam um comportamento menos oscilatório, se comparados com 8 elementos de processamento. Nota-se que, utilizando 2 elementos de processamento, a variação de pontos na direção $x$, considerando fixo o número de pontos em y não implica uma significativa variação do índice de speedup. O speedup apresenta valores acima do esperado para os casos 11 e 16, utilizando-se 8 elementos 


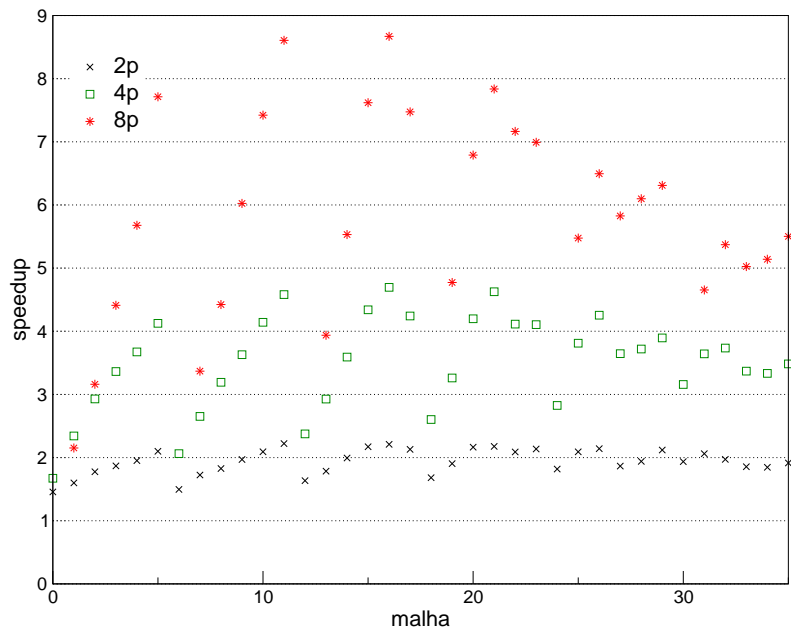

(a) método CS

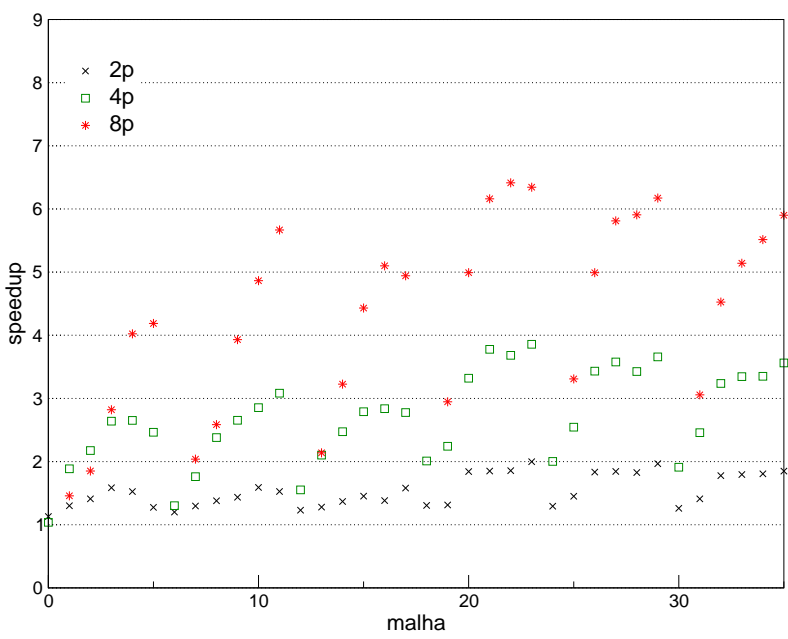

(b) método FAS

Figura 4.6: Speedup dos métodos multigrid paralelizados para o caso II.

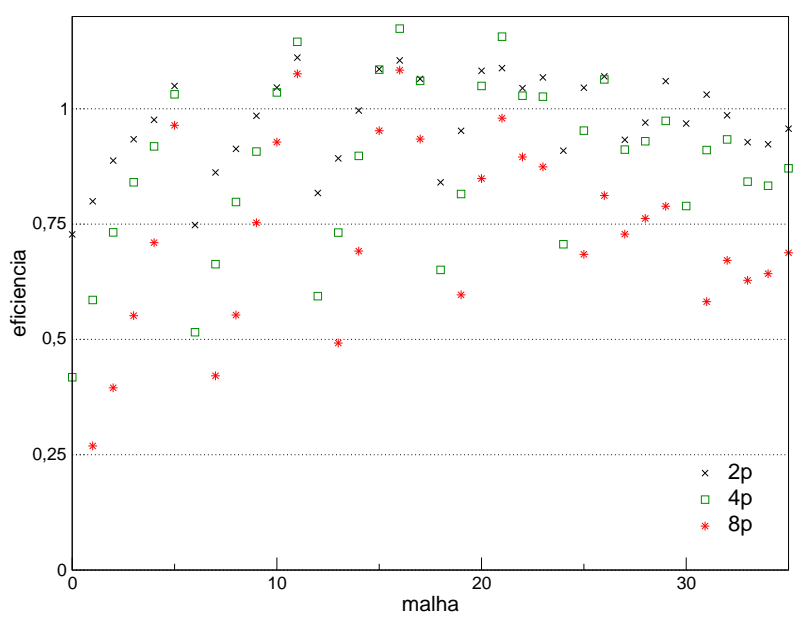

(a) método CS

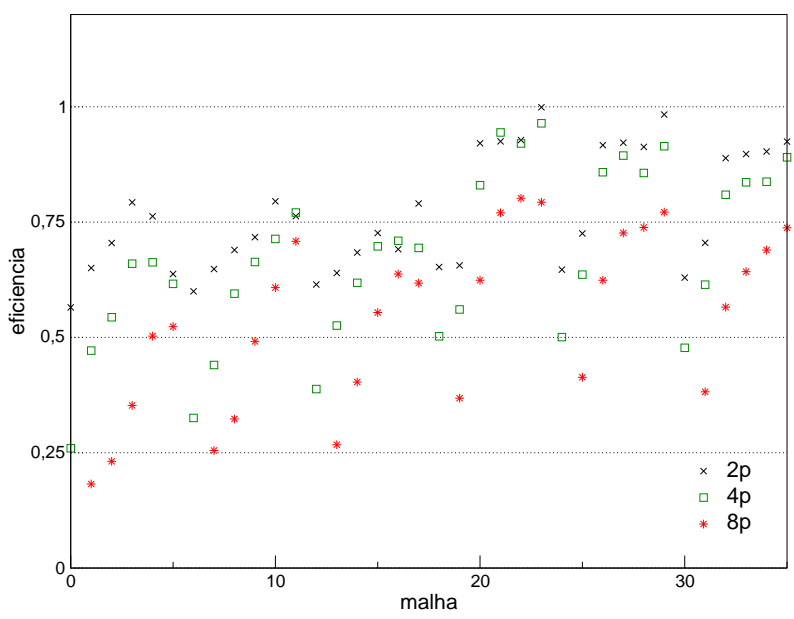

(b) método FAS

Figura 4.7: Eficiência dos métodos multigrid paralelizados para o caso II.

de processamento e para os casos $5,10,11,15,16,17,20,21,22,23$ e 26, utilizando-se 4 elementos de processamento. Os índices de speedup próximos e acima de 2 , considerando 2 elementos de processamento, são observados na grande maioria das malhas testadas.

Por meio de análises voltadas à figura 4.6(b), o método FAS comporta-se de forma similar ao caso I. Em concordância com os resultados anteriores, o crescimento do índice de speedup acompanha o aumento do número de pontos na direção $x$ para a grande parte do conjunto de dados testados. Ganhos de mais de 6 vezes podem ser observados nas malhas intermediárias, com a utilização de 8 elementos de processamento.

É possível afirmar, observando as figuras 4.6(b) e 4.7(b), que o método FAS é eficiente para o caso testado. De forma análoga, nota-se que método CS também é eficiente para todas as malhas testadas de acordo com as figuras 4.6(a) e 4.7(a), apresentado eficiência maior do que 1 em vários casos. 
Tabela 4.2: Eixo nominal - malhas utilizadas - sistemas tri e pentadiagonais.

\begin{tabular}{ccc}
\hline \hline $\mathrm{N}$ & imax & jmax \\
\hline 1 & 1049 & 129 \\
2 & 2073 & 129 \\
3 & 4121 & 129 \\
4 & 1049 & 257 \\
5 & 2073 & 257 \\
6 & 4121 & 257 \\
7 & 1049 & 512 \\
8 & 2073 & 512 \\
9 & 4121 & 512 \\
\hline \hline
\end{tabular}

\subsection{Diferenças compactas de alta ordem paralelizadas}

No sentido de avaliar o desempenho dos algoritmos apresentados na seção 3.2, realiza-se um comparativo entre o código sequencial e os códigos paralelizados, considerando 2,4 e 8 elementos de processamento.

Testes são gerados considerando malhas descritas pela tabela 4.2. Os sistemas tridiagonais utilizados provém do uso de diferenças compactas de alta ordem para estimar as derivadas de primeira e segunda ordem de uma função na direção $x$, que para o presente teste é dada por $g(x)=\operatorname{sen}(x)$. $\mathrm{O}$ espaçamento utilizado é $\Delta y=\Delta x=0.1$.

O tempo de execução apresentado leva em consideração o tempo de computação necessário para se estimar 1000 vezes os valores das derivadas. Essa escolha é justificada uma vez que o tempo necessário para se obtenha a solução do problema é muito pequeno e sendo assim, diferenças significativas entre as estratégias poderiam não ser, de fato, observadas.

Os valores descritos são obtidos por meio de uma média aritmética considerando 5 simulações, na tentativa de se considerar variações de tempo de execução decorrentes de variáveis como a influência do sistema operacional, acesso à memória, entre outros.

Os gráficos 4.8(a) e 4.9(a) apresentam o speedup associado às estratégias de paralelização I e II do cálculo de derivadas de primeira e segunda ordens, respectivamente. De maneira complementar, os gráficos 4.8(b) e 4.9(b) apresentam a eficiência dos métodos em questão.

De forma geral, por meio de análises associadas às figuras 4.8(a) e 4.9(a), é possível notar que entre os casos testados, o aumento no número de pontos nas direções $x$ e $y$ não implica variações significativas em relação aos índices de speedup considerando 2 e 4 elementos de processamento. Além disso, fixando-se o número de elementos de processamento, a diferença entre índices de speedup obtidos pelas duas estratégias é praticamente constante.

Torna-se interessante ressaltar que os melhores resultados são obtidos por meio da segunda versão paralelizada, fato justificado pela diminuição do número de comunicações realizadas em relação a primeira versão e por um melhor aproveitamento dos recursos computacionais. 


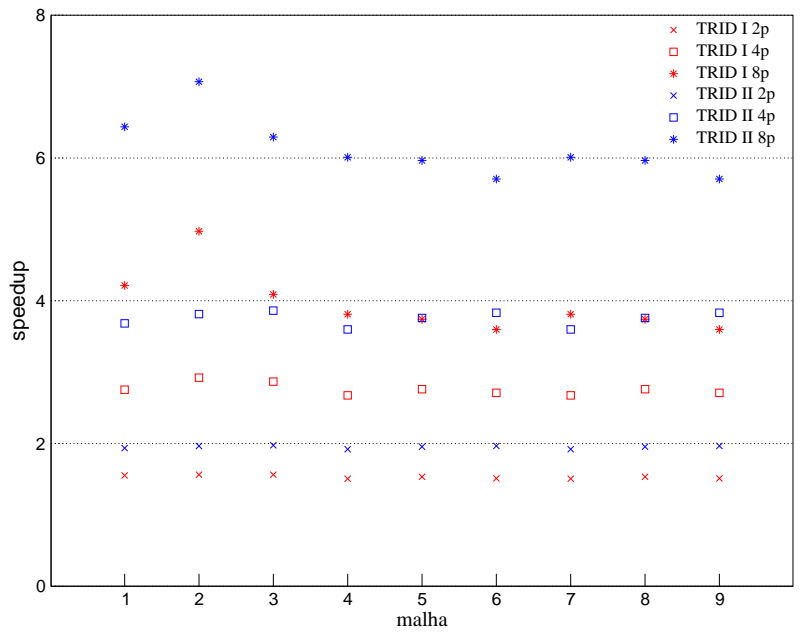

(a) speedup

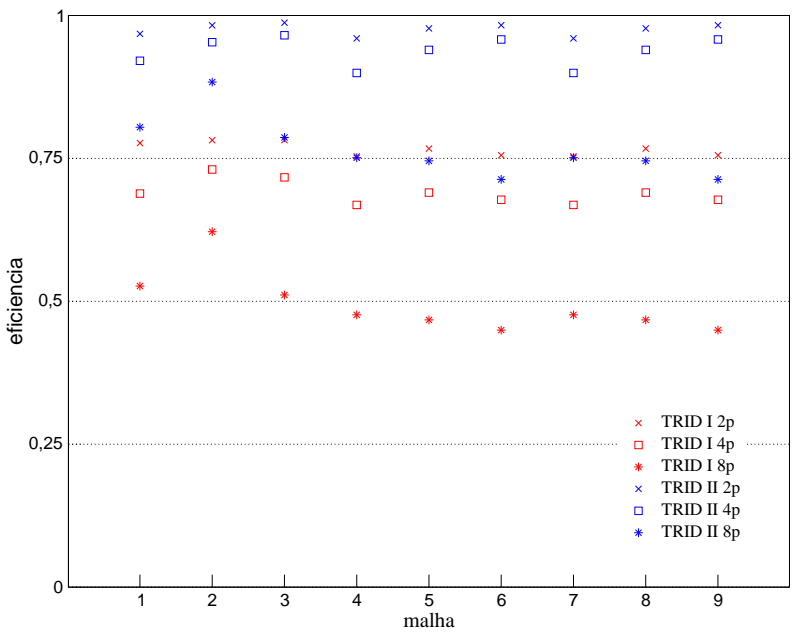

(b) eficiência

Figura 4.8: Speedup e Eficiência referentes ao cálculo da $1^{a}$ derivada.

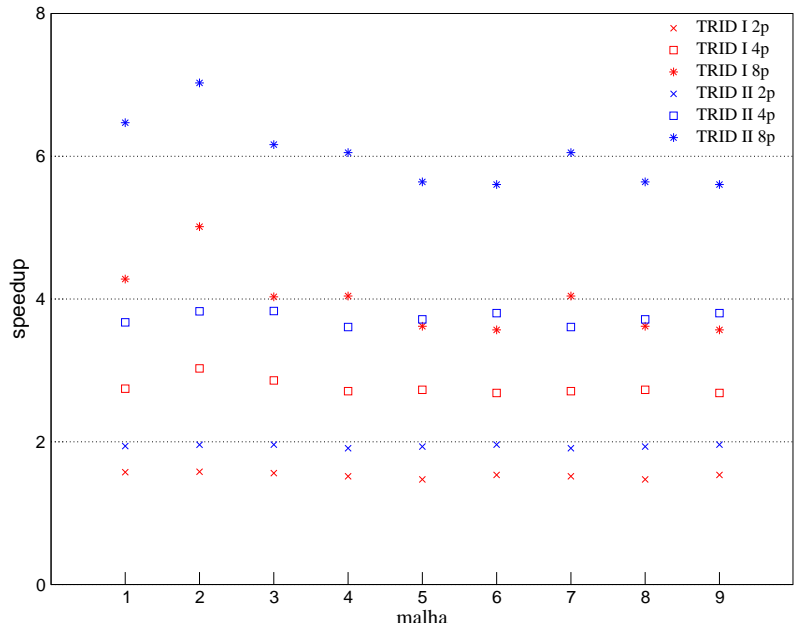

(a) speedup

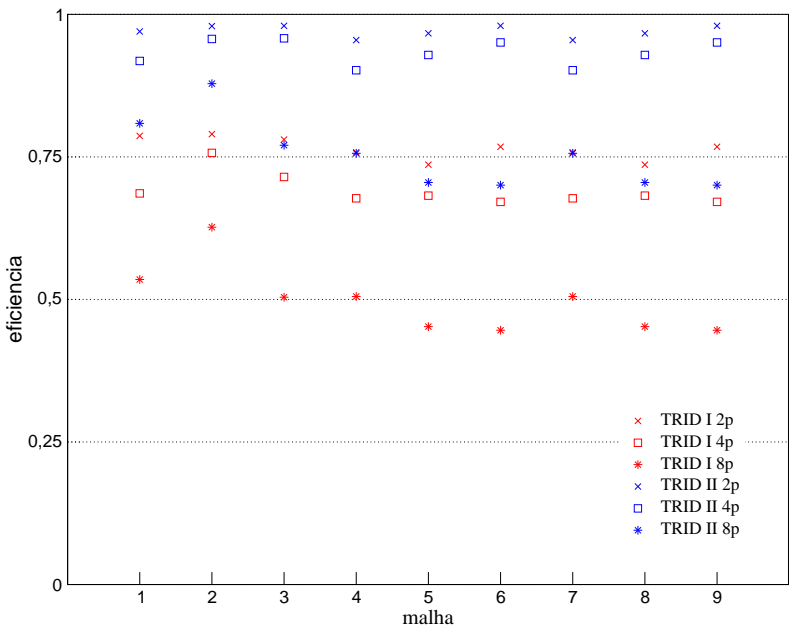

(b) eficiência

Figura 4.9: Speedup e Eficiência referentes ao cálculo da $2^{a}$ derivada.

De maneira complementar, diferenças existentes (mesmo que pequenas) entre o cálculo de aproximações para as derivadas de primeira e segunda ordens podem ser justificadas pela diferença no número de operações aritméticas necessárias para se obter os termos independentes associados aos sistemas lineares em questão.

\subsection{A paralelização na filtragem espacial}

Testes são gerados no sentido de verificar vantagens e desvantagens na aplicação de cada uma das técnicas de paralelização voltadas à aplicação do filtro. O caso teste considerado impõem a aplicação 
do filtro a uma função descontinua $g(x)$, onde

$$
g(x)=\left\{\begin{array}{lll}
0.5 & \text { se } & x \leq a \\
1.5 & \text { se } & x>a
\end{array}\right.
$$

em que $a$ representa a posição onde se encontra a descontinuidade.

O gráfico 4.10(a) apresenta o speedup associado às estratégias de paralelização implementadas, considerando 1000 vezes a aplicação do filtro, em uma função à qual a descontinuidade encontra-se na região de intersecção entre domínios adjacentes. O gráfico de eficiência é descrito pela figura 4.10(b).

Os domínios a serem considerados contemplam as malhas descritas pela tabela 4.2. O espaçamento utilizado é de $\Delta x=\Delta y=0.1$. Assim como nos testes anteriores, os resultados apresentados são gerados por meio de uma média aritmética entre 5 simulações.

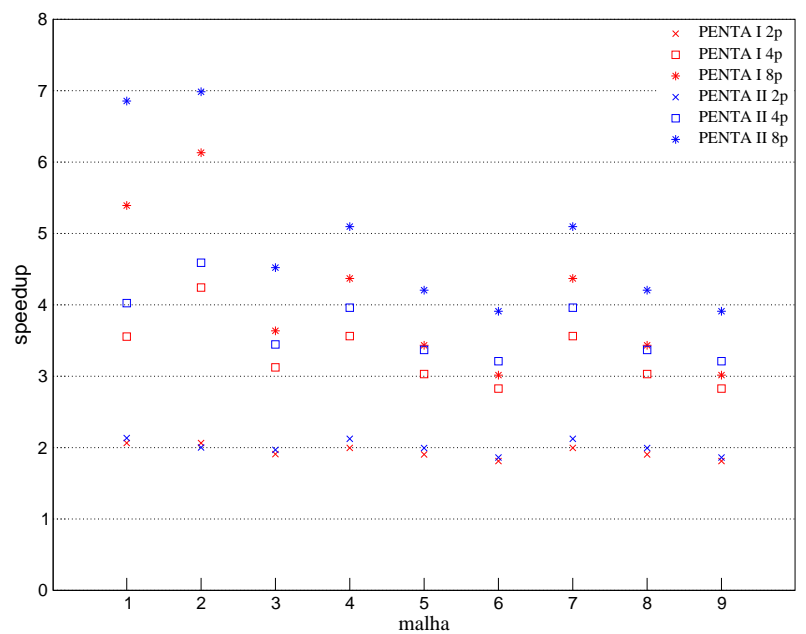

(a) speedup

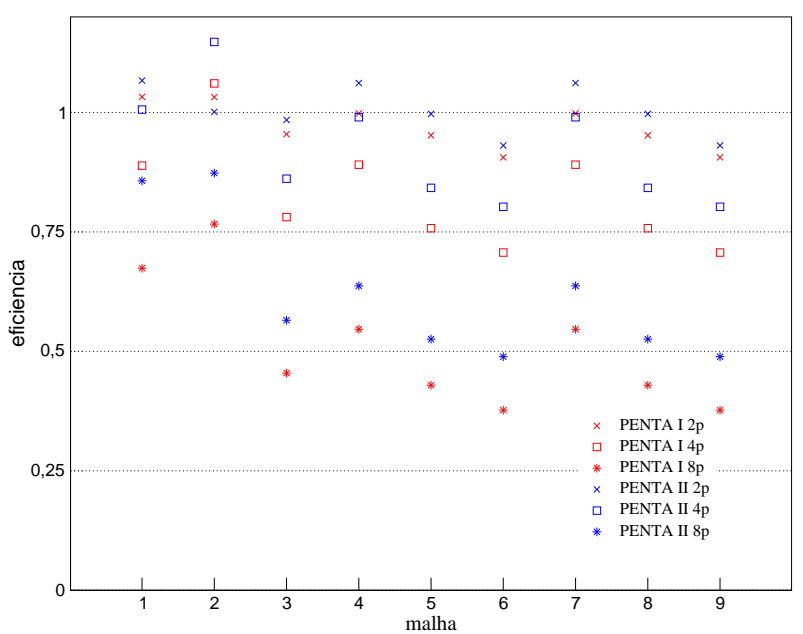

(b) eficiência

Figura 4.10: Speedup e Eficiência referentes a aplicação do filtro.

Por meio de análises voltadas aos gráficos 4.10(a) e 4.10(b), é possível identificar o impacto do número de elementos de processamento frente aos índices de speedup. O aumento do número de pontos, em ambas as direções, demanda um maior tempo de comunicação entre elementos de processamento adjacentes, o que justifica os baixos índices de speedup, notadamente no caso onde utiliza-se 8 elementos de processamento.

Análises voltadas à figura 4.10(b) possibilitam afirmar que há casos em que os índices excedem valores teóricos. Exemplifica-se a afirmação por meio das malhas 1 e 2, com a utilização de 2 elementos de processamento, para ambas as estratégias de paralelização.

A segunda estratégia de paralelização mostrou-se superior a primeira para todos os casos testados. De forma análoga ao algoritmo voltado à geração de aproximações compactas de alta ordem pela segunda estratégia de paralelização, os ganhos obtidos decorrem principalmente da diminuição do número de pontos de comunicação e um maior aproveitamento dos recursos computacionais. No 
entanto, considerando apenas a versão II, faz-se necessário investigar possíveis diferenças existentes entre as soluções obtidas pelo caso sequencial e pelos casos paralelizados.

Para tanto, considera-se a função dada pela equação (4.5) anteriormente citada, com o ponto de descontinuidade pertencente a uma região de intersecção. Toma-se ainda 377 pontos na direção em que o filtro é aplicado utilizando um espaçamento igual à $\Delta x=0.0086875$. A paralelização é realizada utilizando 2 elementos de processamento, por simplicidade.

A figura 4.11 apresenta a diferença absoluta entre a solução obtida pela aplicação do filtro referente à versão sequencial e a gerada por meio da segunda estratégia de paralelização apresentada. A diferença entre a versão sequencial e a primeira estratégia de paralelização é da ordem de $10^{-14}$.

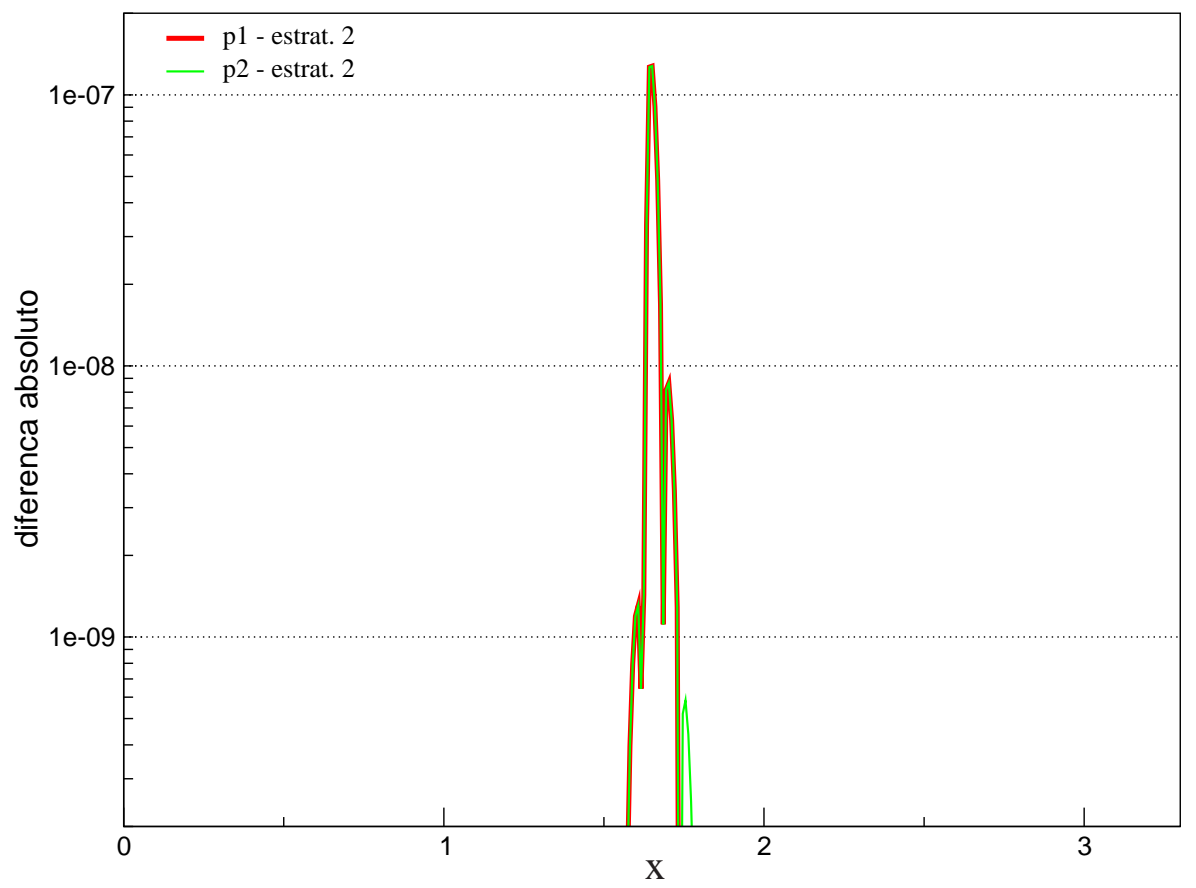

Figura 4.11: Diferença existente entre a aplicação do filtro sequencial e do filtro paralelizado utilizando a estratégia de paralelização II.

Analisando a figura 4.11, é possível perceber uma diferença significativa próxima a região de intersecção entre os domínios adjacentes. Justifica-se essa diferença pelo fato da técnica utilizada para o filtro não alterar os três elementos que compõem os extremos. Neste sentido, por mais que a estratégia de paralelização II seja mais rápida que a estratégia de paralelização I, a magnitude dessa diferença torna a aplicação da segunda técnica inviável.

\subsection{Escoamento de Poiseuille}

\subsubsection{Escoamento de Poiseuille e a Teoria de Estabilidade Linear}

Objetiva-se investigar o comportamento das estratégias de paralelização do método numérico proposto frente à simulação de transição à turbulência em um escoamento de Poiseuille incompressível e 
a teoria de estabilidade linear. Neste sentido, considera-se um domínio retangular, representado pela figura 4.12 .

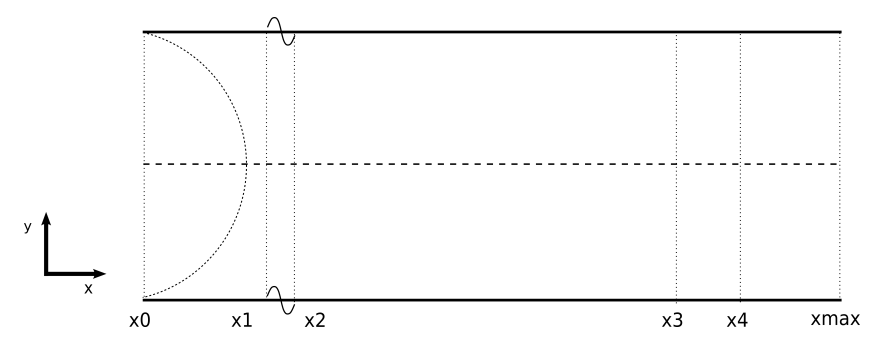

Figura 4.12: Escoamento de Poiseuille - domínio considerado.

O escoamento base, parabólico, é gerado pela função $u(y)=-y^{2}+2 y$. Adota-se $A=0.0005$ como a constante referente à função perturbação. O número de passos de tempo considerados por período de onda é de 128 . Utiliza-se ainda, 32 pontos por comprimento de onda de Tollmien-Schlichting. Define-se ainda $i \max =1433$ e $j \max =81$ como sendo o número de pontos na direção $x$ e $y$, respectivamente. Toma-se $\Delta y=\frac{2}{j \max -1}$ e $\Delta x=\frac{2 \pi}{32 \alpha_{r}}$, sendo $\alpha_{r}$ a parte real do número de onda. O tempo final de simulação é obtido pela aplicação de 42 vezes o passo por período considerado. As demais constantes utilizadas, em função do espaçamento da malha são:

$$
\begin{aligned}
& x_{0}=0, \\
& x_{1}=39 \Delta x, \\
& x_{2}=71 \Delta x, \\
& x_{3}=(i \max -100) \Delta x, \\
& x_{4}=(\text { imax }-40) \Delta x .
\end{aligned}
$$

A tolerância associada ao método multigrid FAS é de $10^{-6}$. O fator de relaxação adotado para o método iterativo por linhas SOR é $r_{2}=1.1$. Em relação ao número de iterações aplicado, toma-se $N_{1}=2, N_{2}=40$ e $N_{3}=1$. No que se refere ao cálculo de derivadas por diferenças compactadas, opta-se pela utilização da segunda estratégia de paralelização.

Os testes gerados consideram os casos neutro, estável e instável, descritos pela tabela 4.3.

Tabela 4.3: Escoamento de Poiseuille - Casos investigados.

\begin{tabular}{ccccc}
\hline \hline & $\operatorname{Re}$ & $\alpha_{r}$ & $\alpha_{i}$ & $\omega$ \\
\hline $\mathrm{A}$ & 5000 & 1.1570000 & 0.0100000 & 0.3300000 \\
$\mathrm{~B}$ & 10000 & 1.0950000 & 0.0000988 & 0.2700000 \\
$\mathrm{C}$ & 10000 & 1.0000000 & -0.0100000 & 0.2375000 \\
\hline \hline
\end{tabular}

Por meio da figura 4.13, é possível verificar a localização dos casos A, B e C estudados em relação à curva neutra, gerada por meio da solução numérica da equação de Orr-Sommerfeld. 


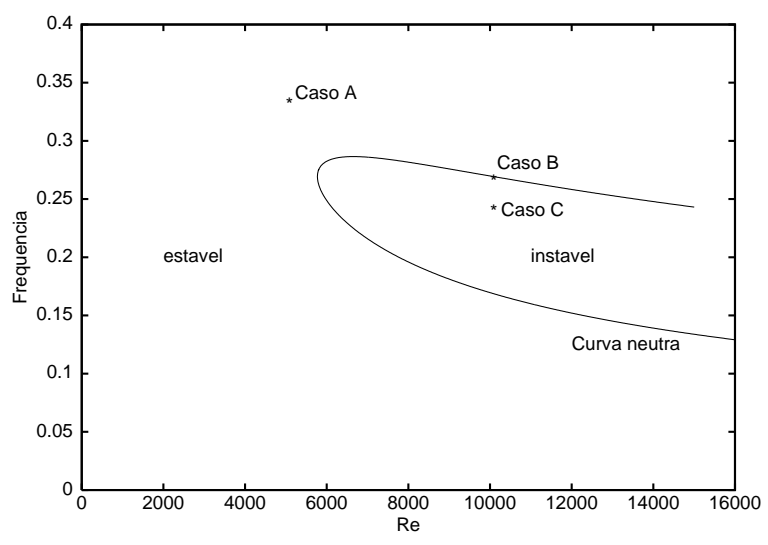

Figura 4.13: Casos considerados frente à curva neutra.

As figuras 4.14(a), 4.14(b) e 4.14(c) apresentam dados qualitativos associados à simulação do caso A utilizando 8 elementos de processamento. Da mesma forma, as figuras 4.15(a), 4.15(b) e 4.15(c) apresentam dados qualitativos associados à simulação do caso neutro. Os dados referentes ao caso instável são apresentados pelas figuras 4.16(a), 4.16(b) e 4.16(c).

Os gráficos 4.17(a) e 4.17(b) apresentam, respectivamente, a velocidade máxima e a taxa de amplificação associada ao primeiro modo de Fourier da velocidade $u$, para cada ponto associado à direção $x$. São apresentados os casos neutro, instável e estável, em concordância com os valores presentes na tabela 4.3.

Observa-se que os valores apresentados pelo gráfico 4.17(b) estão de acordo com dados gerados pela teoria de estabilidade linear. Nota-se que as pequenas oscilações apresentadas decorrem, fundamentalmente, do nível de refinamento da malha considerada. Além disso, justificam-se as oscilações próximas aos extremos devido à introdução de perturbações e a questão da relaminarização do escoamento. Por meio do gráfico 4.17(a) fica claramente evidenciado o comportamento de crescimento, estabilidade e decrescimento da amplitude máxima da perturbação da velocidade $U_{\max }$, para os casos instável, neutro e estável, respectivamente. Os valores associados às taxas de amplificação estão próximos dos valores de referência, presentes na tabela 4.3. Os valores obtidos são apresentados pela tabela 4.4.

Tabela 4.4: Escoamento de Poiseuille - Comparativo realizado utilizando a teoria de estabilidade linear.

\begin{tabular}{cccc}
\hline \hline & $\mathrm{Re}$ & $\alpha_{i}($ LST $)$ & $\alpha_{i}($ Numérico $)$ \\
\hline $\mathrm{A}$ & 5000 & 0.0100000 & 0.0104254 \\
$\mathrm{~B}$ & 10000 & 0.0000988 & 0.0000513 \\
$\mathrm{C}$ & 10000 & -0.0100000 & -0.0111146 \\
\hline \hline
\end{tabular}




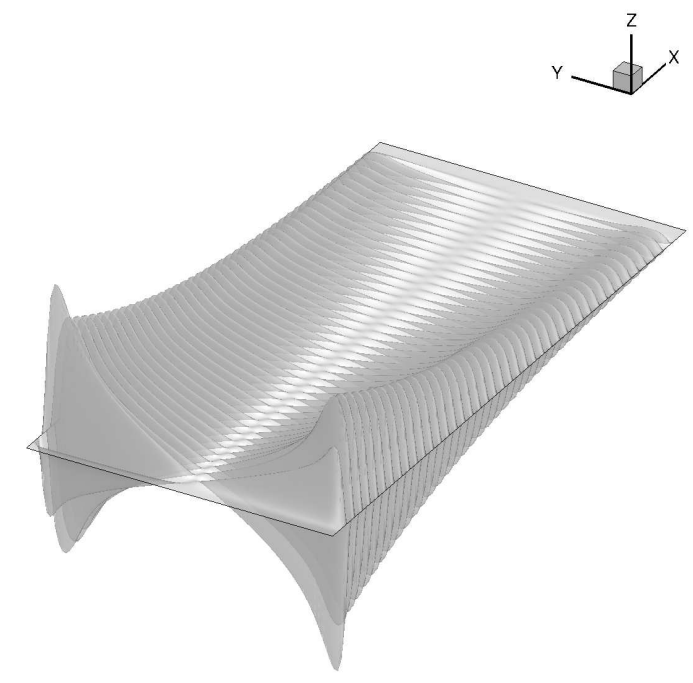

(a) velocidade $u$

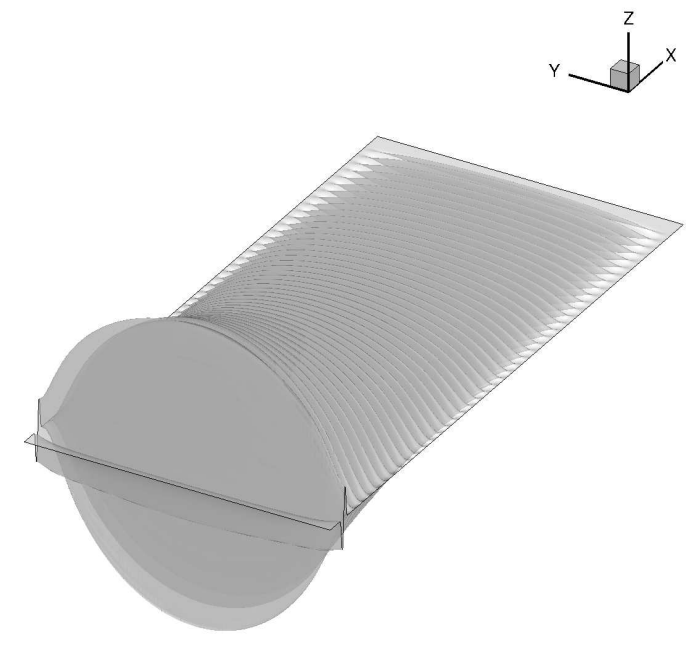

(b) velocidade $\mathrm{v}$

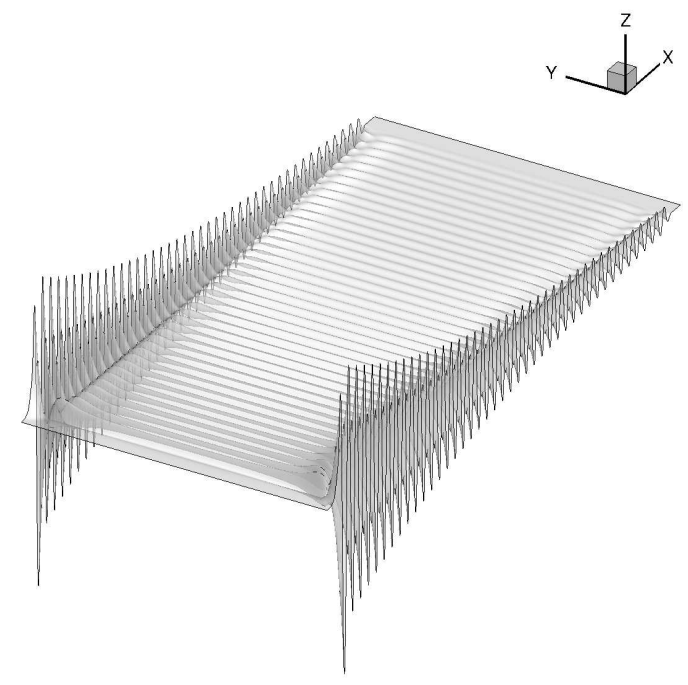

(c) vorticidade

Figura 4.14: Escoamento de Poiseuille - Caso A - dados qualitativos.

\subsubsection{Escoamento de Poiseuille - Comparação entre os métodos multi- grid CS e FAS}

Visando verificar a influência da paralelização frente à simulação do escoamento de Poiseuille em questão, considera-se novamente o caso B, apresentado pela tabela 4.3. O domínio considerado é representado pela figura 4.12. Os mesmos parâmetros, citados na seção anterior, são utilizados. Os testes são gerados considerando um conjunto de 9 malhas, descrito pela tabela 4.5.

Os resultados apresentados consideram ainda, como tempo final de simulação, 19, 30 e 48 vezes o passos por período associados às malhas considerando 665, 1049 e 1433 na direção $x$, respectivamente. De maneira complementar, o método multigrid CS em questão utiliza $r_{1}=0.75$ e $r_{2}=1.1$, como fatores de relaxação associados aos métodos de Jacobi e SOR, respectivamente. No que se 


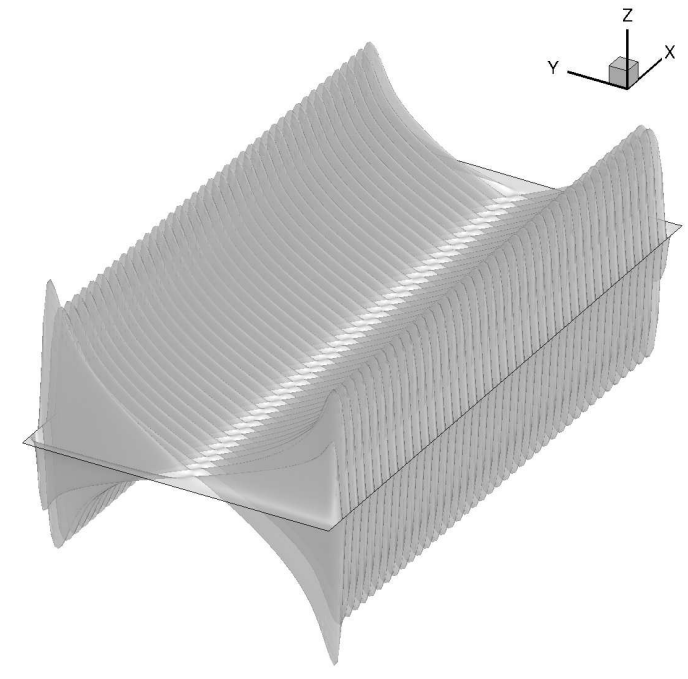

(a) velocidade $u$

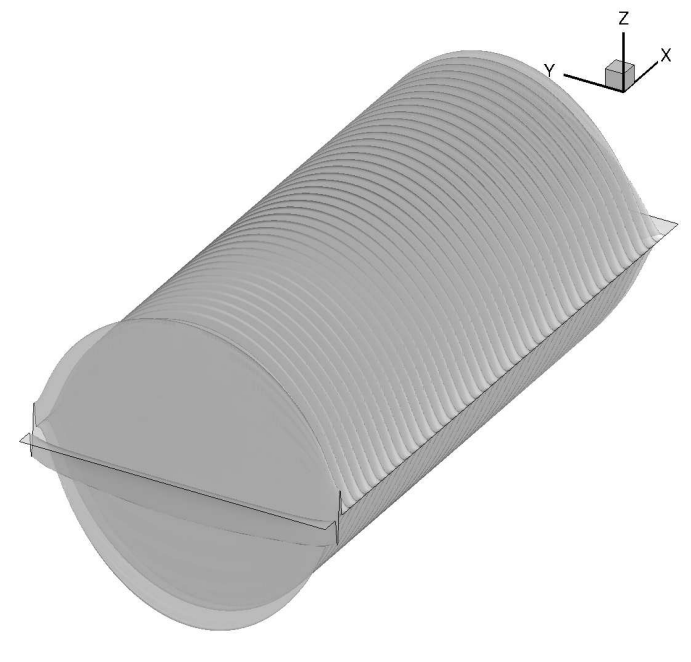

(b) velocidade $\mathrm{v}$

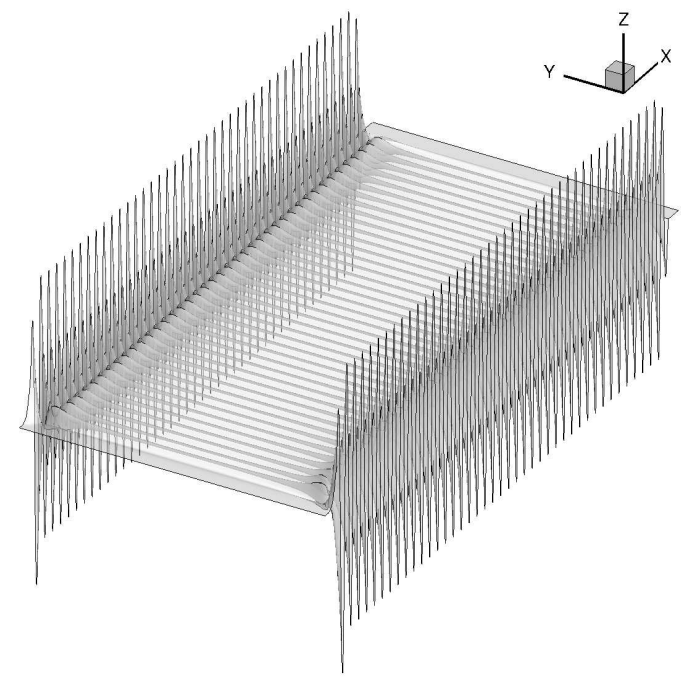

(c) vorticidade

Figura 4.15: Escoamento de Poiseuille - Caso B - dados qualitativos.

Tabela 4.5: Escoamento de Poiseuille - Eixo nominal - malhas utilizadas.

\begin{tabular}{ccc}
\hline \hline $\mathrm{N}$ & imax & jmax \\
\hline 1 & 665 & 49 \\
2 & 665 & 65 \\
3 & 665 & 81 \\
4 & 1049 & 49 \\
5 & 1049 & 65 \\
6 & 1049 & 81 \\
7 & 1433 & 49 \\
8 & 1433 & 65 \\
9 & 1433 & 81 \\
\hline \hline
\end{tabular}




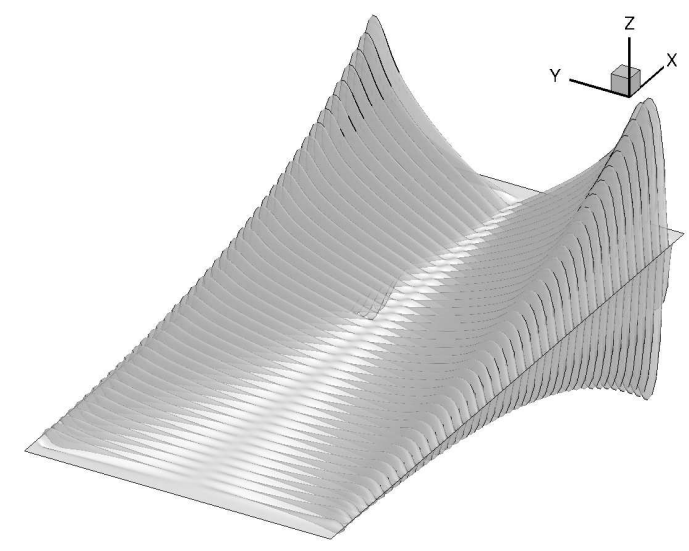

(a) velocidade $u$

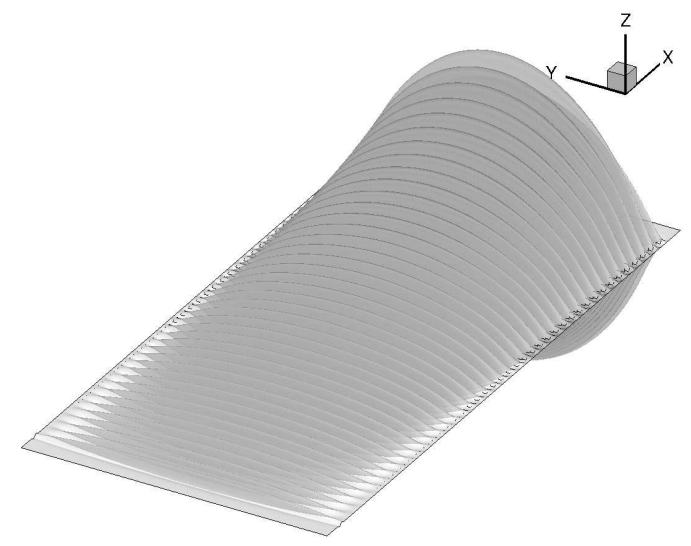

(b) velocidade $\mathrm{v}$

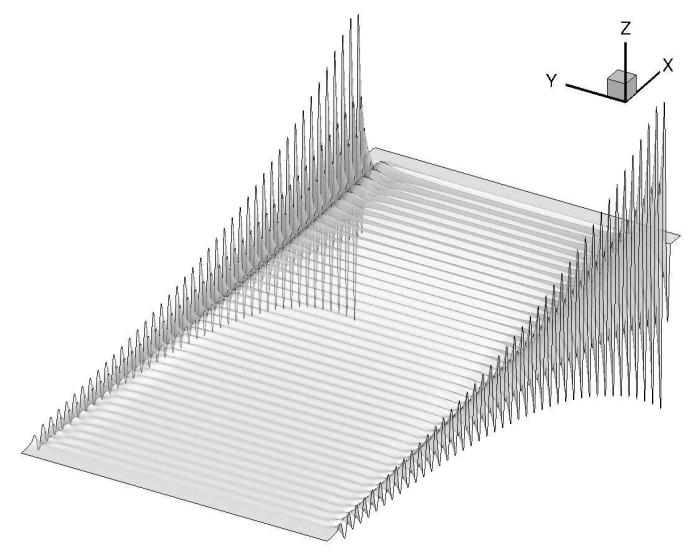

(c) vorticidade

Figura 4.16: Escoamento de Poiseuille - Caso C - dados qualitativos.

refere à paralelização dos métodos voltados a geração de aproximações compactas, opta-se pela utilização da estratégia de paralelização I.

O gráfico 4.18 ilustra o tempo de execução associado à simulação dos 9 casos, apresentados pela tabela 4.5. O teste apresenta o comportamento sequencial dos métodos multigrid CS e FAS.

Em concordância com os resultados obtidos anteriormente, o método multigrid FAS mostrou-se mais rápido que o método $\mathrm{CS}$, para todos os casos considerados. $\mathrm{O}$ aumento do número de pontos em $y$ implica em um crescimento no tempo destinado a execução dos códigos em questão. Ressalta-se que não há sentido em realizar comparativos entre grupos de malhas com número de pontos em $x$ distintos, já que o tempo total de simulação é alterado.

Visando analisar o comportamento dos métodos multigrid paralelizados, as medidas de speedup e eficiência, voltados a estratégia de paralelização I, são apresentadas pelas figuras 4.19(a) e 4.19(b). 


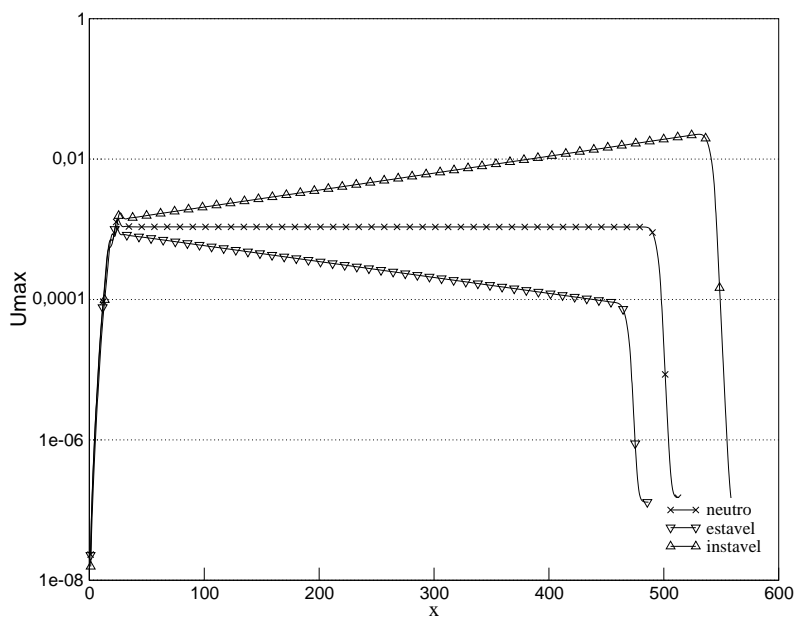

(a) velocidade máxima

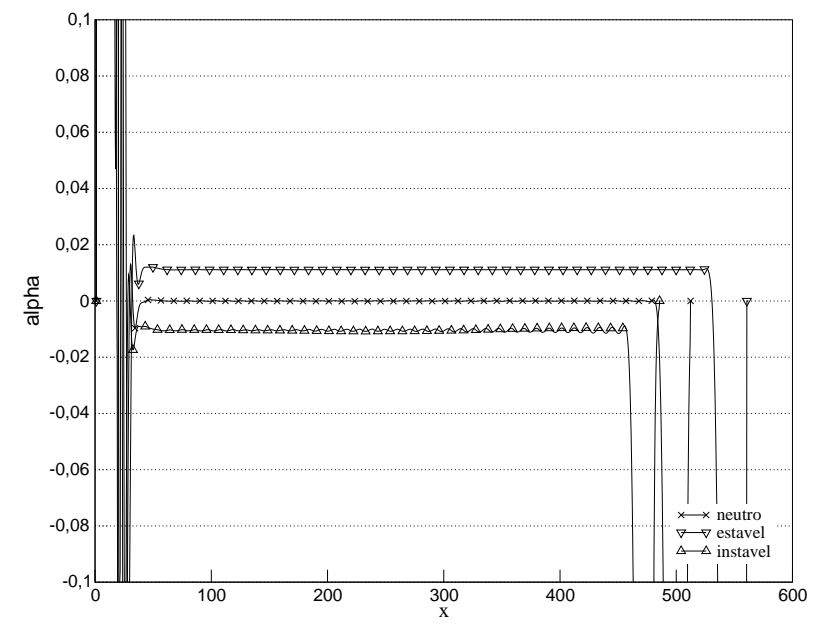

(b) taxa de amplificação

Figura 4.17: Escoamento de Poiseuille - Análise de Fourier - Comportamento referente ao primeiro modo.

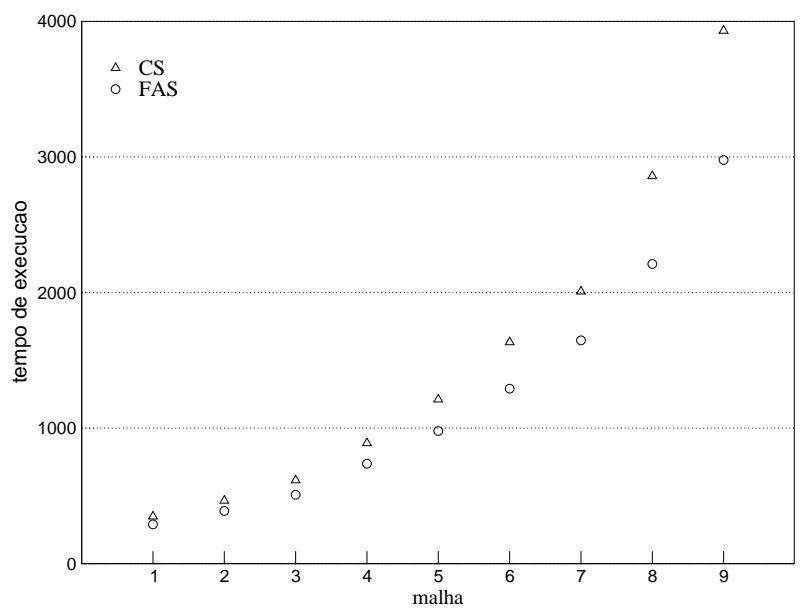

Figura 4.18: Escoamento de Poiseuille - comparativo entre métodos multigrid sequenciais.

É possível perceber uma certa uniformidade dos índices de speedup para ambos os métodos multigrid analisados. Os índices, notadamente com a utilização de 2 e 4 elementos de processamento, apresentam resultados muito positivos. Os melhores índices de speedup, para todos os casos testados, são obtidos por meio da paralelização do método CS. Índices de eficiência próximos ou superiores ao ótimo são obtidos com a utilização de 2 e 4 elementos de processamento.

Além disso, fica novamente evidenciado a diferença entre os índices de speedup entre os métodos multigrid, devido ao impacto referente ao tempo de comunicação gasto pela aplicação do método de Gauss-Seidel, no caso do método multigrid FAS. Por meio de análises voltadas aos gráficos 4.18 e 4.19(a), é possível perceber que o método multigrid CS paralelizado mesmo que mais eficiente que o método FAS paralelizado, demanda um tempo computacional maior. No entanto, esses resultados sugerem uma tendência a utilização do método CS paralelizado, em casos onde o número de pontos cresce. No que se refere à estratégia de paralelização II voltada a geração de aproximações compactas 


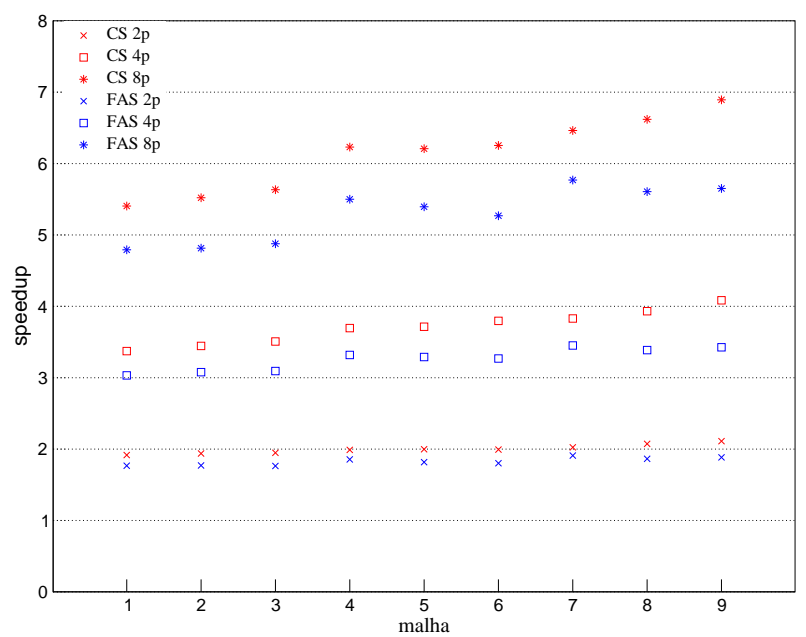

(a) speedup

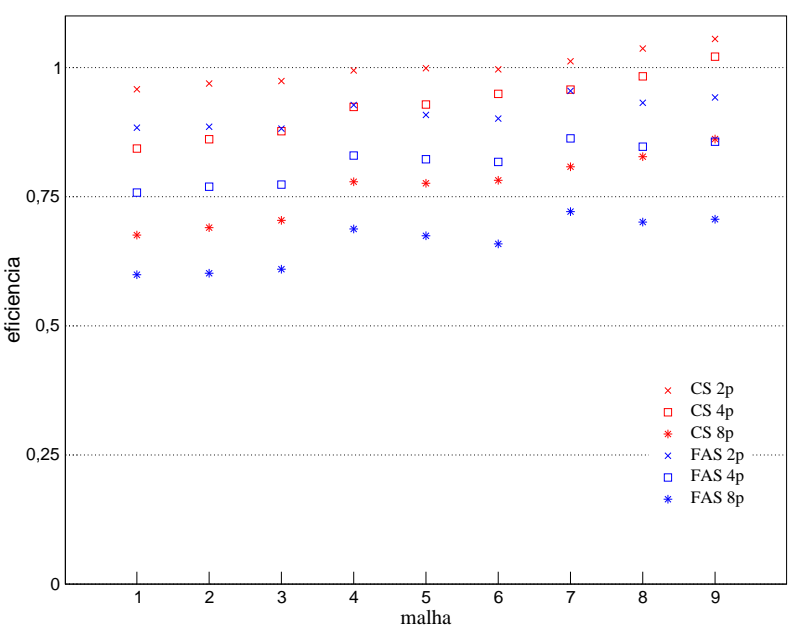

(b) eficiência

Figura 4.19: Escoamento de Poiseuille - comparativo entre métodos multigrid utilizando a estratégia I de paralelização de aproximação de diferenças compactas.

de alta ordem, as medidas de speedup e eficiência são apresentadas, respectivamente, pelas figuras 4.20(a) e 4.20(b).

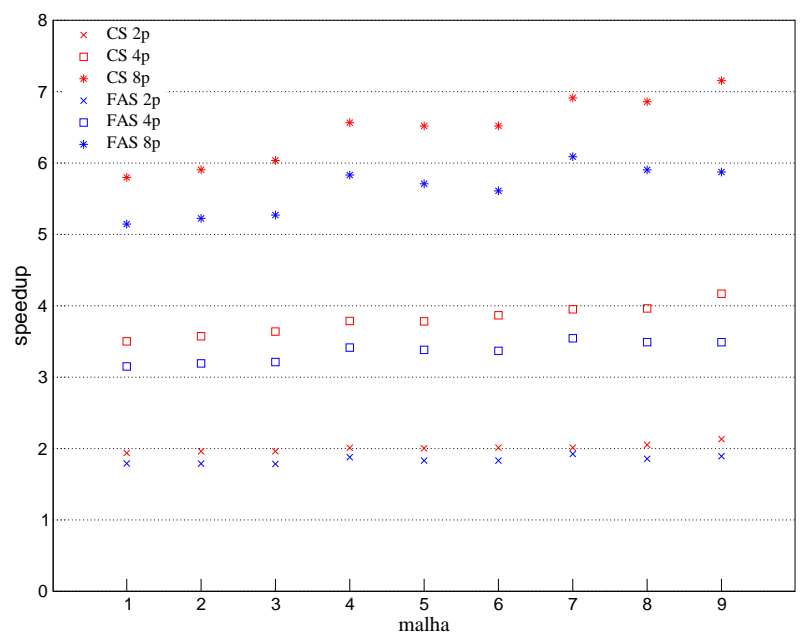

(a) speedup

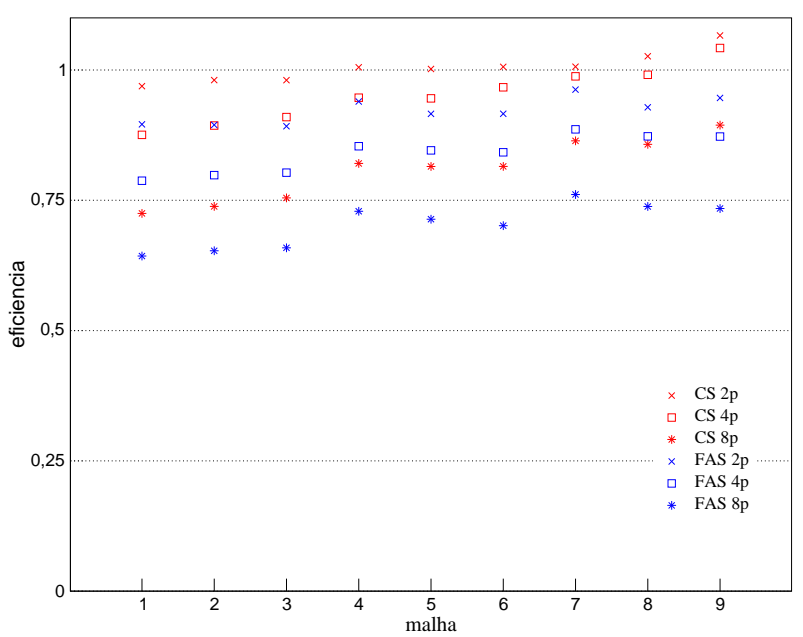

(b) eficiência

Figura 4.20: Escoamento de Poiseuille - comparativo entre métodos multigrid utilizando a estratégia II de paralelização de aproximação de diferenças compactas.

Os resultados em questão, considerando a segunda estratégia de paralelização, apresentam resultados análogos ao caso anterior.

\subsubsection{Escoamento de Poiseuille - Comparação entre estratégias de pa- ralelização para diferenças compactas}

Faz-se necessário ainda, realizar comparativos entre as estratégias de paralelização voltadas a aproximação de derivadas, por diferenças compactas de alta ordem. Neste sentido, as medidas de 
speedup e eficiência, considerando apenas o método multigrid FAS, são apresentadas pelas figuras 4.21(a) e 4.21(b).

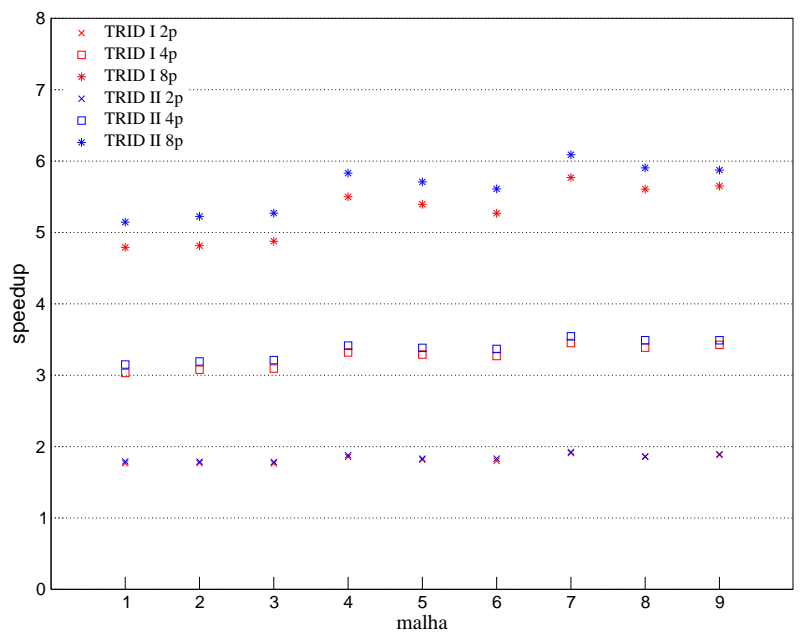

(a) speedup

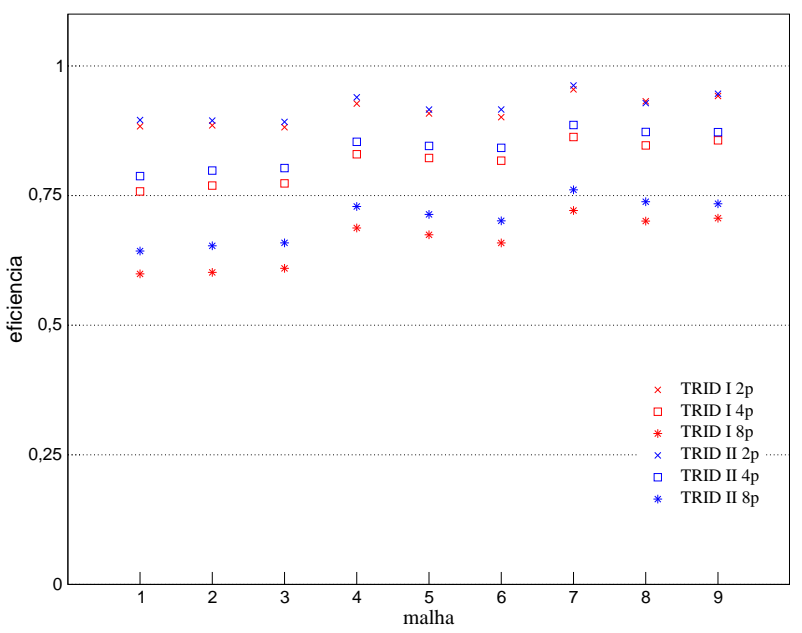

(b) eficiência

Figura 4.21: Escoamento de Poiseuille - comparativo entre estratégias de paralelização de aproximação de diferenças compactas utilizando o método multigrid FAS.

De acordo com as figuras 4.21(a) e 4.21(b), a segunda estratégia de paralelização mostrou-se superior a primeira, para todos os casos testados. Ressalta-se que o aumento no número de elementos de processamento implica um aumento da diferença entre os índices de speedup e eficiência das estratégias de paralelização em questão. 


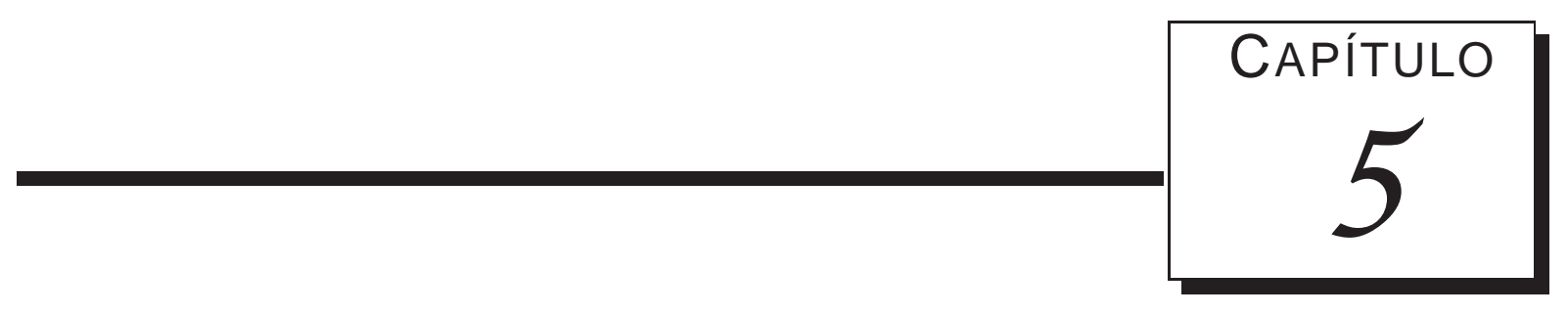

\section{Conclusões e trabalhos futuros}

\subsection{Síntese do trabalho realizado}

O presente trabalho de pesquisa visa, inicialmente, apresentar um estudo voltado à resolução numérica paralelizada das equações de Navier-Stokes incompressíveis, utilizando a formulação vorticidade-velocidade. Os resultados obtidos são então comparados com a teoria de estabilidade linear.

No sentido de implementar e avaliar o desempenho de um código numérico voltado à simulação de escoamentos incompressíveis, estudos são realizados no sentido de:

- implementar e verificar códigos computacionais paralelizados responsáveis pela resolução da equação de Poisson bidimensional, através de métodos multigrid;

- implementar e verificar códigos computacionais paralelizados responsáveis pela aproximação de derivadas utilizando diferenças compactas de alta ordem;

- implementar e verificar códigos computacionais paralelizados responsáveis pela aplicação de um filtro computacional;

Através dos resultados obtidos, torna-se importante avaliar como as estratégias de paralelização comportam-se frente à simulação de um escoamento bidimensional incompressível. Neste sentido, são realizados testes e análises voltados à aplicação dessas estratégias para simular um escoamento de Poiseuille.

\subsection{Considerações}

No que se refere ao comparativo realizado entre os métodos multigrid em questão, a paralelização dos processos pode ser considerada como uma estratégia eficiente, principalmente quando o número de variáveis envolvidas é grande. 
Na maioria dos casos observados, o método multigrid FAS apresenta os melhores índices de tempo de execução. Em contrapartida, os melhores índices de speedup e eficiência são obtidos com a utilização do método multigrid CS. Justifica-se essa diferença uma vez que o número de pontos de comunicação associados ao método multigrid FAS é, de fato, maior que no caso do método multigrid CS.

Duas estratégias de paralelização voltadas à geração de aproximações de derivadas espaciais por diferenças compactas são avaliadas. A primeira utiliza conceitos de pipeline, enquanto a segunda explora a decomposição de domínio utilizada por métodos multigrid. A segunda estratégia de paralelização apresenta o melhor resultado. Justifica-se tal fato devido a questões de redução do número de pontos de comunicação e um melhor aproveitamento dos recursos computacionais.

As mesmas estratégias de paralelização são aplicadas na solução de sistemas pentadiagonais, utilizada no filtro numérico. Verifica-se que, apesar da estratégia II gerar resultados com um tempo de execução inferior, a presença dos coeficientes iguais a 1, nos extremos do sistema pentadiagonal em questão, implica diferenças significativas entre as estratégias sequencial e paralelizada. Essa diferença inviabiliza a utilização da estratégia de paralelização II no código implementado.

De forma análoga aos casos analisados, o uso de estratégias paralelizadas e a aplicação de métodos multigrid voltados à resolução numérica das equações de Navier-Stokes mostram-se muito viáveis. A escolha pela aplicação do método multigrid FAS no contexto da simulação de transição à turbulência é justificada uma vez que os índices de tempo de execução para as malhas intermediárias são significativamente melhores, mesmo apresentando índices de speedup e eficiência menores que os apresentados pelo método multigrid CS. É possível afirmar ainda que, um aumento do número de pontos a serem considerados, torne o método multigrid CS paralelizado mais competitivo. Em relação à paralelização do cálculo de derivadas espaciais voltada à solução numérica das equações de Navier-Stokes, o melhor aproveitamento dos recursos computacionais e a redução de pontos de comunicação, obtidos pela segunda estratégia de paralelização confirmam, novamente, a viabilidade na utilização dessa estratégia.

\subsection{Trabalhos futuros}

Sugere-se como tópicos importantes voltados à continuidade do projeto de pesquisa:

- estudo aprofundado voltado à influência dos pontos de comunicação entre subdomínios adjacentes frente aos índices de speedup e eficiência dos códigos multigrid em questão;

- estudo da influência dos diversos parâmetros associados aos métodos multigrid frente às estratégias de paralelização;

- desenvolvimento e/ou implementação de estratégias de paralelização eficientes no que se refere à aplicação do filtro computacional; 
- implementação de um código paralelizado $3 D$ no sentido de simular o fenômeno de transição à turbulência; 


\section{Referências Bibliográficas}

Abdullah, A. R. e Ali, N. H. M., 1996. A comparative study of parallel strategies for the solution of elliptic PDE's. Parallel algorithms and Applications, vol. 10, pp. 93-103.

Ali, N. M. e Fu, N. K., 2008. A new iterative elliptic PDE solver on a distributed PC cluster. pdcat, vol. 41, pp. 47-53.

Brandt, A., 1977. Multilevel adaptative solutions to boundary values problems. Mathematics of Computation, vol. 31, pp. 333-390.

Briggs, W. L., Helson, V. E., e McCormick, S. F., 2000. A Multigrid Tutorial. SIAM, 2 edition.

Brown, M. J. e Ingber, M. S., 2002. Parallelization of a vorticity formulation for the analysis of incompressible viscous fluid flows. International Journal for Numerical Methods in Fluids, vol. 39, pp. 979-999.

Buckeridge, S. e Scheichl, R., 2010. Parallel geometric multigrid for global weather prediction. Numerical Linear Algebra with Applications, vol. 17, pp. 325-342.

de Angeli, J. P., Valli, A. M. P., Jr, N. C. R., e de Souza, A. F., 2003. Finite difference simulation of the navier-stokes equations using paralle distributed computing. In Proceedings of the 15th Symposium on Computer Architecture and High Performance Computing.

de Velde, E. F. V., 1994. Concurrent Scientific Computing. Springer-Verlag.

Fasel, H. F., Rist, U., e Konzelmann, U., 1990. Numerical investigation of the three-dimensional development in boundary-layer transition. AIAA, vol. 28, pp. 29-37.

Ferziger, J. H. e Peric, M., 1997. Computational Methods for Fluid Dynamics. Springer-Verlag Berlin Heidelberg New York.

Ge, Y., 2010. Multigrid method and fourth-order compact difference discretization scheme with unequal meshsizes for 3D Poisson equation. Journal of Computational Physics, vol. 229, pp. 6381-6391. 
Gupta, M. M., Kouatchou, J., e Zhang, J., 1997. Comparison of second- and fourth-order discretizations for multigrid Poisson solvers. J. Computational Physics, vol. 132, pp. 226-232.

Henniger, R., Obrist, D., e Kleiser, L., 2010. High-order accurate solution of the incompressible Navier-Stokes equations on massively parallel computers. Journal of Computational Physics, vol. 229 , pp. 3543-3572.

Hirsh, R. S., 1975. High order accurate difference solutions of fluid mechanics problem by a compact differencing technique. Journal of Computational Physics, vol. 19, pp. 90-109.

John, V. e Tobiska, L., 2000. Numerical performance of smoothers in coupled multigrid methods for the parallel solution of the incompressible Navier-Stokes equations. International Journal for Numerical Methods in Fluids, vol. 33, pp. 453-473.

Kloker, M., 1998. A robust high-tesolution split-type compact FD scheme for spatial Direct Numerical Simulation of boundary-layer transition. Applied Scientific Research, vol. 59, pp. 353-377.

Lele, S., 1992. Compact finite difference schemes with spectral-like resolution. J. Computational Physics, vol. 103, pp. 16-42.

Mahesh, K., 1998. A family of high order finite difference schemes with good spectral resolution. $J$. Computational Physics, vol. 145, pp. 332-358.

Meitz, H. L., 1996. Numerical investigation of suction in a trasitional flat-plate boundary layer. $\mathrm{PhD}$ thesis, The University of Arizona.

Mendonça, M. T., 2003. Estabilidade de escoamentos laminares: teoria linear, chapter 5, pp. 01-50. ABCM - ETT 2000.

Schlichting, H., 1979. Boundary-Layer Theory. McGraw-Hill, IncH.

Simens, M. P., Jimenez, J., Hoyas, S., e Mizuno, Y., 2009. A high-resolution code for turbulent boundary-layers. Journal of Computational Physics, vol. 228, pp. 4218-4231.

Souza, L. F., 2003. Instabilidade Centrífuga e Transição para Turbulência em Escoamentos Laminares sobre Superfícies Côncavas. PhD thesis, Instituto Tecnológico de Aeronáutica.

Souza, L. F., Mendonça, M. T., e Medeiros, M. A. F., 2005. The advantages of using high-order finite differences schemes in laminar-turbulent transition studies. International Journal for Numerical Methods in Fluids, vol. 48, pp. 565-592.

Spitaleri, R. M., 2000. Full-FAS multigrid grid generation algorithms. Applied Numerical Math., vol. 32, pp. 483-494. 
Sterk, M. e Trobec, R., 2003. Parallel performances of a multigrid poisson solver. In Proceedings of the Second International Symposium on Parallel and Distributed Computing.

Wang, Y. e Zhang, J., 2009. Sixth order compact scheme combined with multigrid method and extrapolation technique for $2 \mathrm{~d}$ poisson equation. Journal of Computational Physics, vol. 228, pp. 137-146.

Wray, A. e Hussaini, M. Y., 1994. Highly accurate compact methods and boundary conditions. Proc. Royal Soc. London, vol. A 392, pp. 373-389.

Zhang, J., 1996. Acceleration of five-point red-black Gauss-Seidel in multigrid for Poisson equation. Applied Math. and Comp., vol. 80, pp. 73-93.

Zhang, J., 1997. Residual scaling techniques in multigrid, I: equivalence proof. Applied Math. and Comp., vol. 80, pp. 283-303.

Zhang, J., 2002. Multigrid method and fourth-order compact scheme for 2d Poisson equation with unequal mesh-size discretization. Journal of Computational Physics, vol. 179, pp. 170-179. 


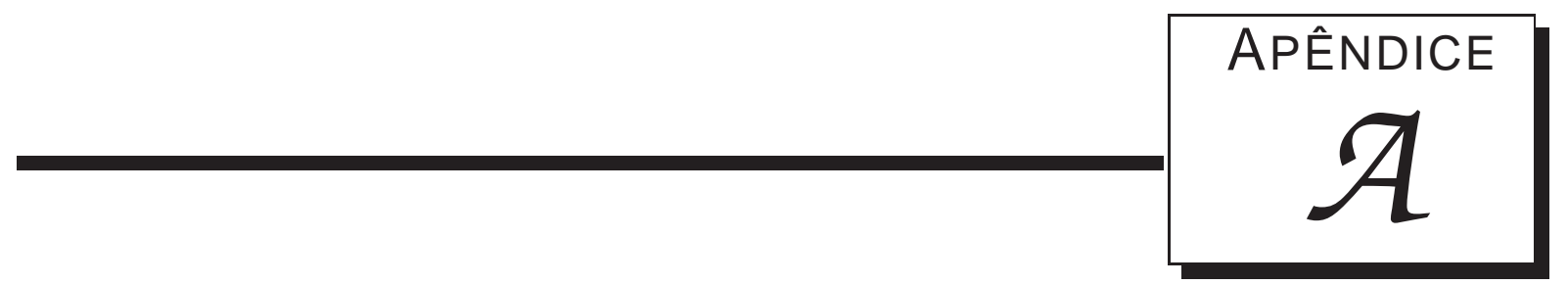

\section{Dados quantitativos}

No presente capítulo estão dispostas tabelas contendo dados quantitativos referentes aos testes de desempenho dos métodos multigrid CS e FAS. São apresentadas também tabelas referentes à análise de desempenho dos códigos implementados frente à simulação do escoamento de Poiseuille bidimensional. A sigla EP é utilizada para representar o número de elementos de processamento utilizados. 
Tabela A.1: Método multigrid CS - Caso I - 1p.

\begin{tabular}{cccccc}
\hline \hline imax & jmax & Nx & ciclos V & erro & tempo \\
\hline 153 & 33 & 153 & 58 & $0.2844176 \mathrm{E}-08$ & $0.7043099 \mathrm{E}-01$ \\
281 & 33 & 281 & 57 & $0.2459446 \mathrm{E}-08$ & $0.1270810 \mathrm{E}+00$ \\
537 & 33 & 537 & 57 & $0.2775856 \mathrm{E}-08$ & $0.2412859 \mathrm{E}+00$ \\
1049 & 33 & 1049 & 58 & $0.2679146 \mathrm{E}-08$ & $0.4806795 \mathrm{E}+00$ \\
2073 & 33 & 2073 & 58 & $0.2774848 \mathrm{E}-08$ & $0.9596733 \mathrm{E}+00$ \\
4121 & 33 & 4121 & 53 & $0.2013411 \mathrm{E}-08$ & $0.1925152 \mathrm{E}+01$ \\
153 & 65 & 153 & 65 & $0.8061173 \mathrm{E}-09$ & $0.1579603 \mathrm{E}+00$ \\
281 & 65 & 281 & 66 & $0.8891500 \mathrm{E}-09$ & $0.2965326 \mathrm{E}+00$ \\
537 & 65 & 537 & 66 & $0.8304413 \mathrm{E}-09$ & $0.5680597 \mathrm{E}+00$ \\
1049 & 65 & 1049 & 64 & $0.8318646 \mathrm{E}-09$ & $0.1123068 \mathrm{E}+01$ \\
2073 & 65 & 2073 & 65 & $0.9232179 \mathrm{E}-09$ & $0.2392976 \mathrm{E}+01$ \\
4121 & 65 & 4121 & 67 & $0.7866724 \mathrm{E}-09$ & $0.5682900 \mathrm{E}+01$ \\
153 & 129 & 153 & 74 & $0.2442924 \mathrm{E}-09$ & $0.3589399 \mathrm{E}+00$ \\
281 & 129 & 281 & 73 & $0.2585376 \mathrm{E}-09$ & $0.6600391 \mathrm{E}+00$ \\
537 & 129 & 537 & 74 & $0.2278322 \mathrm{E}-09$ & $0.1372201 \mathrm{E}+01$ \\
1049 & 129 & 1049 & 74 & $0.2492990 \mathrm{E}-09$ & $0.3019817 \mathrm{E}+01$ \\
2073 & 129 & 2073 & 70 & $0.2514289 \mathrm{E}-09$ & $0.6366489 \mathrm{E}+01$ \\
4121 & 129 & 4121 & 73 & $0.2200941 \mathrm{E}-09$ & $0.1385502 \mathrm{E}+02$ \\
153 & 257 & 153 & 79 & $0.6454482 \mathrm{E}-10$ & $0.7864891 \mathrm{E}+00$ \\
281 & 257 & 281 & 82 & $0.5986707 \mathrm{E}-10$ & $0.1596763 \mathrm{E}+01$ \\
537 & 257 & 537 & 82 & $0.5838656 \mathrm{E}-10$ & $0.3511599 \mathrm{E}+01$ \\
1049 & 257 & 1049 & 81 & $0.6565407 \mathrm{E}-10$ & $0.7630306 \mathrm{E}+01$ \\
2073 & 257 & 2073 & 82 & $0.6546408 \mathrm{E}-10$ & $0.1620499 \mathrm{E}+02$ \\
4121 & 257 & 4121 & 77 & $0.6206300 \mathrm{E}-10$ & $0.3271795 \mathrm{E}+02$ \\
153 & 513 & 153 & 83 & $0.1693767 \mathrm{E}-10$ & $0.1754744 \mathrm{E}+01$ \\
281 & 513 & 281 & 86 & $0.1760122 \mathrm{E}-10$ & $0.3879975 \mathrm{E}+01$ \\
537 & 513 & 537 & 89 & $0.1715124 \mathrm{E}-10$ & $0.8631905 \mathrm{E}+01$ \\
1049 & 513 & 1049 & 89 & $0.1761130 \mathrm{E}-10$ & $0.1779247 \mathrm{E}+02$ \\
2073 & 513 & 2073 & 89 & $0.1531310 \mathrm{E}-10$ & $0.3665717 \mathrm{E}+02$ \\
4121 & 513 & 4121 & 90 & $0.1616794 \mathrm{E}-10$ & $0.7953674 \mathrm{E}+02$ \\
153 & 1025 & 153 & 87 & $0.4226760 \mathrm{E}-11$ & $0.4262843 \mathrm{E}+01$ \\
281 & 1025 & 281 & 91 & $0.3779532 \mathrm{E}-11$ & $0.9253128 \mathrm{E}+01$ \\
537 & 1025 & 537 & 94 & $0.4112759 \mathrm{E}-11$ & $0.1940111 \mathrm{E}+02$ \\
1049 & 1025 & 1049 & 108 & $0.1972958 \mathrm{E}-06$ & $0.4500501 \mathrm{E}+02$ \\
2073 & 1025 & 2073 & 170 & $0.3100115 \mathrm{E}-06$ & $0.1435817 \mathrm{E}+03$ \\
4121 & 1025 & 4121 & 192 & $0.3764637 \mathrm{E}-06$ & $0.3452161 \mathrm{E}+03$ \\
\hline \hline
\end{tabular}


Tabela A.2: Método multigrid CS - Caso I - 2p.

\begin{tabular}{cccccc}
\hline \hline imax & jmax & Nx & ciclos V & erro & tempo \\
\hline 153 & 33 & 89 & 58 & $0.2844176 \mathrm{E}-08$ & $0.5111763 \mathrm{E}-01$ \\
281 & 33 & 153 & 57 & $0.2459446 \mathrm{E}-08$ & $0.8162266 \mathrm{E}-01$ \\
537 & 33 & 281 & 57 & $0.2775856 \mathrm{E}-08$ & $0.1378736 \mathrm{E}+00$ \\
1049 & 33 & 537 & 58 & $0.2679146 \mathrm{E}-08$ & $0.2575144 \mathrm{E}+00$ \\
2073 & 33 & 1049 & 58 & $0.2774848 \mathrm{E}-08$ & $0.4921474 \mathrm{E}+00$ \\
4121 & 33 & 2073 & 53 & $0.2013411 \mathrm{E}-08$ & $0.9017977 \mathrm{E}+00$ \\
153 & 65 & 89 & 65 & $0.8061172 \mathrm{E}-09$ & $0.1052486 \mathrm{E}+00$ \\
281 & 65 & 153 & 66 & $0.8891495 \mathrm{E}-09$ & $0.1731103 \mathrm{E}+00$ \\
537 & 65 & 281 & 66 & $0.8304413 \mathrm{E}-09$ & $0.3091515 \mathrm{E}+00$ \\
1049 & 65 & 537 & 64 & $0.8318645 \mathrm{E}-09$ & $0.5657802 \mathrm{E}+00$ \\
2073 & 65 & 1049 & 65 & $0.9232180 \mathrm{E}-09$ & $0.1159133 \mathrm{E}+01$ \\
4121 & 65 & 2073 & 67 & $0.7866722 \mathrm{E}-09$ & $0.2608358 \mathrm{E}+01$ \\
153 & 129 & 89 & 74 & $0.2442903 \mathrm{E}-09$ & $0.2262177 \mathrm{E}+00$ \\
281 & 129 & 153 & 73 & $0.2585339 \mathrm{E}-09$ & $0.3715222 \mathrm{E}+00$ \\
537 & 129 & 281 & 74 & $0.2278325 \mathrm{E}-09$ & $0.6880400 \mathrm{E}+00$ \\
1049 & 129 & 537 & 74 & $0.2492993 \mathrm{E}-09$ & $0.1396188 \mathrm{E}+01$ \\
2073 & 129 & 1049 & 70 & $0.2514289 \mathrm{E}-09$ & $0.2973922 \mathrm{E}+01$ \\
4121 & 129 & 2073 & 73 & $0.2200941 \mathrm{E}-09$ & $0.6716499 \mathrm{E}+01$ \\
153 & 257 & 89 & 79 & $0.6454589 \mathrm{E}-10$ & $0.4640200 \mathrm{E}+00$ \\
281 & 257 & 153 & 82 & $0.5986295 \mathrm{E}-10$ & $0.8371181 \mathrm{E}+00$ \\
537 & 257 & 281 & 82 & $0.5838578 \mathrm{E}-10$ & $0.1622457 \mathrm{E}+01$ \\
1049 & 257 & 537 & 81 & $0.6565393 \mathrm{E}-10$ & $0.3607859 \mathrm{E}+01$ \\
2073 & 257 & 1049 & 82 & $0.6546411 \mathrm{E}-10$ & $0.7816553 \mathrm{E}+01$ \\
4121 & 257 & 2073 & 77 & $0.6206300 \mathrm{E}-10$ & $0.1539512 \mathrm{E}+02$ \\
153 & 513 & 89 & 83 & $0.1693912 \mathrm{E}-10$ & $0.9650988 \mathrm{E}+00$ \\
281 & 513 & 153 & 86 & $0.1760026 \mathrm{E}-10$ & $0.1857939 \mathrm{E}+01$ \\
537 & 513 & 281 & 89 & $0.1715018 \mathrm{E}-10$ & $0.4167379 \mathrm{E}+01$ \\
1049 & 513 & 537 & 89 & $0.1762114 \mathrm{E}-10$ & $0.8910122 \mathrm{E}+01$ \\
2073 & 513 & 1049 & 89 & $0.1690063 \mathrm{E}-10$ & $0.1839128 \mathrm{E}+02$ \\
4121 & 513 & 2073 & 90 & $0.1616177 \mathrm{E}-10$ & $0.3741274 \mathrm{E}+02$ \\
153 & 1025 & 89 & 87 & $0.4227386 \mathrm{E}-11$ & $0.2236660 \mathrm{E}+01$ \\
281 & 1025 & 153 & 91 & $0.3779498 \mathrm{E}-11$ & $0.4628065 \mathrm{E}+01$ \\
537 & 1025 & 281 & 94 & $0.4132493 \mathrm{E}-11$ & $0.9669900 \mathrm{E}+01$ \\
1049 & 1025 & 537 & 116 & $0.9900439 \mathrm{E}-07$ & $0.2465726 \mathrm{E}+02$ \\
2073 & 1025 & 1049 & 175 & $0.2423311 \mathrm{E}-06$ & $0.7340037 \mathrm{E}+02$ \\
4121 & 1025 & 2073 & 196 & $0.3131190 \mathrm{E}-06$ & $0.1677173 \mathrm{E}+03$ \\
\hline \hline
\end{tabular}


Tabela A.3: Método multigrid CS - Caso I - 4p.

\begin{tabular}{cccccc}
\hline \hline imax & jmax & Nx & ciclos V & erro & tempo \\
\hline 153 & 33 & 57 & 58 & $0.2844176 \mathrm{E}-08$ & $0.4135975 \mathrm{E}-01$ \\
281 & 33 & 89 & 57 & $0.2459446 \mathrm{E}-08$ & $0.5617315 \mathrm{E}-01$ \\
537 & 33 & 153 & 57 & $0.2775856 \mathrm{E}-08$ & $0.8697984 \mathrm{E}-01$ \\
1049 & 33 & 281 & 58 & $0.2679146 \mathrm{E}-08$ & $0.1470678 \mathrm{E}+00$ \\
2073 & 33 & 537 & 58 & $0.2774848 \mathrm{E}-08$ & $0.2637791 \mathrm{E}+00$ \\
4121 & 33 & 1049 & 53 & $0.2013411 \mathrm{E}-08$ & $0.4565562 \mathrm{E}+00$ \\
153 & 65 & 57 & 65 & $0.8061173 \mathrm{E}-09$ & $0.7823589 \mathrm{E}-01$ \\
281 & 65 & 89 & 66 & $0.8891499 \mathrm{E}-09$ & $0.1119682 \mathrm{E}+00$ \\
537 & 65 & 153 & 66 & $0.8304412 \mathrm{E}-09$ & $0.1829431 \mathrm{E}+00$ \\
1049 & 65 & 281 & 64 & $0.8318645 \mathrm{E}-09$ & $0.3091235 \mathrm{E}+00$ \\
2073 & 65 & 537 & 65 & $0.9232178 \mathrm{E}-09$ & $0.5796739 \mathrm{E}+00$ \\
4121 & 65 & 1049 & 67 & $0.7866723 \mathrm{E}-09$ & $0.1222824 \mathrm{E}+01$ \\
153 & 129 & 57 & 74 & $0.2442884 \mathrm{E}-09$ & $0.1564326 \mathrm{E}+00$ \\
281 & 129 & 89 & 73 & $0.2585230 \mathrm{E}-09$ & $0.2314426 \mathrm{E}+00$ \\
537 & 129 & 153 & 74 & $0.2278326 \mathrm{E}-09$ & $0.3812473 \mathrm{E}+00$ \\
1049 & 129 & 281 & 74 & $0.2492991 \mathrm{E}-09$ & $0.6951606 \mathrm{E}+00$ \\
2073 & 129 & 537 & 70 & $0.2514288 \mathrm{E}-09$ & $0.1365262 \mathrm{E}+01$ \\
4121 & 129 & 1049 & 73 & $0.2200941 \mathrm{E}-09$ & $0.3397711 \mathrm{E}+01$ \\
153 & 257 & 57 & 79 & $0.6455606 \mathrm{E}-10$ & $0.3037477 \mathrm{E}+00$ \\
281 & 257 & 89 & 82 & $0.5985784 \mathrm{E}-10$ & $0.4825373 \mathrm{E}+00$ \\
537 & 257 & 153 & 82 & $0.5837857 \mathrm{E}-10$ & $0.8401246 \mathrm{E}+00$ \\
1049 & 257 & 281 & 81 & $0.6565468 \mathrm{E}-10$ & $0.1678183 \mathrm{E}+01$ \\
2073 & 257 & 537 & 82 & $0.6546395 \mathrm{E}-10$ & $0.3971407 \mathrm{E}+01$ \\
4121 & 257 & 1049 & 77 & $0.6206312 \mathrm{E}-10$ & $0.8007545 \mathrm{E}+01$ \\
153 & 513 & 57 & 83 & $0.1694575 \mathrm{E}-10$ & $0.6124638 \mathrm{E}+00$ \\
281 & 513 & 89 & 86 & $0.1760026 \mathrm{E}-10$ & $0.1010599 \mathrm{E}+01$ \\
537 & 513 & 153 & 89 & $0.1714778 \mathrm{E}-10$ & $0.2024428 \mathrm{E}+01$ \\
1049 & 513 & 281 & 89 & $0.1763618 \mathrm{E}-10$ & $0.4559301 \mathrm{E}+01$ \\
2073 & 513 & 537 & 89 & $0.1986014 \mathrm{E}-10$ & $0.9579814 \mathrm{E}+01$ \\
4121 & 513 & 1049 & 90 & $0.1614769 \mathrm{E}-10$ & $0.2007557 \mathrm{E}+02$ \\
153 & 1025 & 57 & 87 & $0.4229672 \mathrm{E}-11$ & $0.1350663 \mathrm{E}+01$ \\
281 & 1025 & 89 & 91 & $0.3779949 \mathrm{E}-11$ & $0.2553950 \mathrm{E}+01$ \\
537 & 1025 & 153 & 94 & $0.4180135 \mathrm{E}-11$ & $0.5222570 \mathrm{E}+01$ \\
1049 & 1025 & 281 & 118 & $0.9925943 \mathrm{E}-07$ & $0.1340632 \mathrm{E}+02$ \\
2073 & 1025 & 537 & 176 & $0.2508677 \mathrm{E}-06$ & $0.4090315 \mathrm{E}+02$ \\
4121 & 1025 & 1049 & 197 & $0.3069574 \mathrm{E}-06$ & $0.9189866 \mathrm{E}+02$ \\
\hline \hline & & & & &
\end{tabular}


Tabela A.4: Método multigrid CS - Caso I - 8p.

\begin{tabular}{cccccc}
\hline \hline imax & jmax & Nx & ciclos V & erro & tempo \\
\hline 281 & 33 & 57 & 57 & $0.2459446 \mathrm{E}-08$ & $0.5175114 \mathrm{E}-01$ \\
537 & 33 & 89 & 57 & $0.2775856 \mathrm{E}-08$ & $0.6571975 \mathrm{E}-01$ \\
1049 & 33 & 153 & 58 & $0.2679146 \mathrm{E}-08$ & $0.9975123 \mathrm{E}-01$ \\
2073 & 33 & 281 & 58 & $0.2774848 \mathrm{E}-08$ & $0.1553163 \mathrm{E}+00$ \\
4121 & 33 & 537 & 53 & $0.2013411 \mathrm{E}-08$ & $0.2563625 \mathrm{E}+00$ \\
281 & 65 & 57 & 66 & $0.8891499 \mathrm{E}-09$ & $0.9041286 \mathrm{E}-01$ \\
537 & 65 & 89 & 66 & $0.8304414 \mathrm{E}-09$ & $0.1229137 \mathrm{E}+00$ \\
1049 & 65 & 153 & 64 & $0.8318646 \mathrm{E}-09$ & $0.1849485 \mathrm{E}+00$ \\
2073 & 65 & 281 & 65 & $0.9232180 \mathrm{E}-09$ & $0.3181589 \mathrm{E}+00$ \\
4121 & 65 & 537 & 67 & $0.7866723 \mathrm{E}-09$ & $0.6452436 \mathrm{E}+00$ \\
281 & 129 & 57 & 73 & $0.2585080 \mathrm{E}-09$ & $0.1635331 \mathrm{E}+00$ \\
537 & 129 & 89 & 74 & $0.2278313 \mathrm{E}-09$ & $0.2374060 \mathrm{E}+00$ \\
1049 & 129 & 153 & 74 & $0.2492967 \mathrm{E}-09$ & $0.3937769 \mathrm{E}+00$ \\
2073 & 129 & 281 & 70 & $0.2514291 \mathrm{E}-09$ & $0.7265795 \mathrm{E}+00$ \\
4121 & 129 & 537 & 73 & $0.2200940 \mathrm{E}-09$ & $0.1924935 \mathrm{E}+01$ \\
281 & 257 & 57 & 82 & $0.5986743 \mathrm{E}-10$ & $0.3392688 \mathrm{E}+00$ \\
537 & 257 & 89 & 82 & $0.5836649 \mathrm{E}-10$ & $0.5087477 \mathrm{E}+00$ \\
1049 & 257 & 153 & 81 & $0.6565071 \mathrm{E}-10$ & $0.9728144 \mathrm{E}+00$ \\
2073 & 257 & 281 & 82 & $0.6546294 \mathrm{E}-10$ & $0.2279882 \mathrm{E}+01$ \\
4121 & 257 & 537 & 77 & $0.6206303 \mathrm{E}-10$ & $0.4711313 \mathrm{E}+01$ \\
281 & 513 & 57 & 86 & $0.1760960 \mathrm{E}-10$ & $0.7029543 \mathrm{E}+00$ \\
537 & 513 & 89 & 89 & $0.1714484 \mathrm{E}-10$ & $0.1325138 \mathrm{E}+01$ \\
1049 & 513 & 153 & 89 & $0.1769328 \mathrm{E}-10$ & $0.2747737 \mathrm{E}+01$ \\
2073 & 513 & 281 & 89 & $0.2846597 \mathrm{E}-10$ & $0.5807154 \mathrm{E}+01$ \\
4121 & 513 & 537 & 90 & $0.1612677 \mathrm{E}-10$ & $0.1220135 \mathrm{E}+02$ \\
281 & 1025 & 57 & 91 & $0.3783397 \mathrm{E}-11$ & $0.1960980 \mathrm{E}+01$ \\
537 & 1025 & 89 & 94 & $0.4501323 \mathrm{E}-11$ & $0.3575964 \mathrm{E}+01$ \\
1049 & 1025 & 153 & 121 & $0.1110769 \mathrm{E}-06$ & $0.8934121 \mathrm{E}+01$ \\
2073 & 1025 & 281 & 179 & $0.2516264 \mathrm{E}-06$ & $0.2629994 \mathrm{E}+02$ \\
4121 & 1025 & 537 & 198 & $0.3205552 \mathrm{E}-06$ & $0.5825197 \mathrm{E}+02$ \\
\hline \hline
\end{tabular}


Tabela A.5: Método multigrid FAS - Caso I - 1p.

\begin{tabular}{cccccc}
\hline \hline imax & jmax & Nx & ciclos V & erro & tempo \\
\hline 153 & 33 & 153 & 10 & $0.1788950 \mathrm{E}-07$ & $0.1667780 \mathrm{E}-01$ \\
281 & 33 & 281 & 10 & $0.1373653 \mathrm{E}-07$ & $0.3051454 \mathrm{E}-01$ \\
537 & 33 & 537 & 10 & $0.1372825 \mathrm{E}-07$ & $0.5860150 \mathrm{E}-01$ \\
1049 & 33 & 1049 & 10 & $0.1652574 \mathrm{E}-07$ & $0.1146683 \mathrm{E}+00$ \\
2073 & 33 & 2073 & 10 & $0.1784924 \mathrm{E}-07$ & $0.2286113 \mathrm{E}+00$ \\
4121 & 33 & 4121 & 8 & $0.1372619 \mathrm{E}-06$ & $0.3793232 \mathrm{E}+00$ \\
153 & 65 & 153 & 11 & $0.1613619 \mathrm{E}-08$ & $0.3637898 \mathrm{E}-01$ \\
281 & 65 & 281 & 11 & $0.2090061 \mathrm{E}-08$ & $0.6709176 \mathrm{E}-01$ \\
537 & 65 & 537 & 11 & $0.1985300 \mathrm{E}-08$ & $0.1289470 \mathrm{E}+00$ \\
1049 & 65 & 1049 & 11 & $0.1324769 \mathrm{E}-08$ & $0.2543249 \mathrm{E}+00$ \\
2073 & 65 & 2073 & 11 & $0.1790293 \mathrm{E}-08$ & $0.5167814 \mathrm{E}+00$ \\
4121 & 65 & 4121 & 11 & $0.2239945 \mathrm{E}-08$ & $0.1085786 \mathrm{E}+01$ \\
153 & 129 & 153 & 12 & $0.2645337 \mathrm{E}-09$ & $0.7813400 \mathrm{E}-01$ \\
281 & 129 & 281 & 12 & $0.2466013 \mathrm{E}-09$ & $0.1447753 \mathrm{E}+00$ \\
537 & 129 & 537 & 12 & $0.2574284 \mathrm{E}-09$ & $0.2828190 \mathrm{E}+00$ \\
1049 & 129 & 1049 & 12 & $0.2845872 \mathrm{E}-09$ & $0.5832468 \mathrm{E}+00$ \\
2073 & 129 & 2073 & 12 & $0.1348017 \mathrm{E}-09$ & $0.1252311 \mathrm{E}+01$ \\
4121 & 129 & 4121 & 12 & $0.2061644 \mathrm{E}-09$ & $0.2602043 \mathrm{E}+01$ \\
153 & 257 & 153 & 13 & $0.2182299 \mathrm{E}-10$ & $0.1712301 \mathrm{E}+00$ \\
281 & 257 & 281 & 13 & $0.6018356 \mathrm{E}-09$ & $0.3231413 \mathrm{E}+00$ \\
537 & 257 & 537 & 13 & $0.5842831 \mathrm{E}-07$ & $0.6732720 \mathrm{E}+00$ \\
1049 & 257 & 1049 & 14 & $0.5485998 \mathrm{E}-07$ & $0.1557286 \mathrm{E}+01$ \\
2073 & 257 & 2073 & 14 & $0.5727361 \mathrm{E}-07$ & $0.3278933 \mathrm{E}+01$ \\
4121 & 257 & 4121 & 14 & $0.6468116 \mathrm{E}-07$ & $0.6966621 \mathrm{E}+01$ \\
153 & 513 & 153 & 13 & $0.1123111 \mathrm{E}-10$ & $0.3544278 \mathrm{E}+00$ \\
281 & 513 & 281 & 15 & $0.2293893 \mathrm{E}-07$ & $0.8092115 \mathrm{E}+00$ \\
537 & 513 & 537 & 28 & $0.2130019 \mathrm{E}-06$ & $0.3165084 \mathrm{E}+01$ \\
1049 & 513 & 1049 & 41 & $0.5085840 \mathrm{E}-06$ & $0.9500881 \mathrm{E}+01$ \\
2073 & 513 & 2073 & 46 & $0.5695329 \mathrm{E}-06$ & $0.2182208 \mathrm{E}+02$ \\
4121 & 513 & 4121 & 46 & $0.5257602 \mathrm{E}-06$ & $0.4717283 \mathrm{E}+02$ \\
153 & 1025 & 153 & 13 & $0.5918960 \mathrm{E}-11$ & $0.7745298 \mathrm{E}+00$ \\
281 & 1025 & 281 & 17 & $0.2342419 \mathrm{E}-07$ & $0.2008483 \mathrm{E}+01$ \\
537 & 1025 & 537 & 44 & $0.1884764 \mathrm{E}-06$ & $0.1054214 \mathrm{E}+02$ \\
1049 & 1025 & 1049 & 100 & $0.4923801 \mathrm{E}-06$ & $0.4788460 \mathrm{E}+02$ \\
2073 & 1025 & 2073 & 157 & $0.7533959 \mathrm{E}-06$ & $0.1524180 \mathrm{E}+03$ \\
4121 & 1025 & 4121 & 177 & $0.8952898 \mathrm{E}-06$ & $0.3691300 \mathrm{E}+03$ \\
\hline \hline
\end{tabular}


Tabela A.6: Método multigrid FAS - Caso I - 2p.

\begin{tabular}{cccccc}
\hline \hline imax & jmax & Nx & ciclos V & erro & tempo \\
\hline 153 & 33 & 89 & 14 & $0.5371892 \mathrm{E}-07$ & $0.1654744 \mathrm{E}-01$ \\
281 & 33 & 153 & 14 & $0.4790446 \mathrm{E}-07$ & $0.2500278 \mathrm{E}-01$ \\
537 & 33 & 281 & 14 & $0.5944006 \mathrm{E}-07$ & $0.4411727 \mathrm{E}-01$ \\
1049 & 33 & 537 & 13 & $0.4614163 \mathrm{E}-07$ & $0.7763648 \mathrm{E}-01$ \\
2073 & 33 & 1049 & 13 & $0.8115951 \mathrm{E}-07$ & $0.1502402 \mathrm{E}+00$ \\
4121 & 33 & 2073 & 14 & $0.5695361 \mathrm{E}-07$ & $0.3178918 \mathrm{E}+00$ \\
153 & 65 & 89 & 16 & $0.2372479 \mathrm{E}-07$ & $0.3374332 \mathrm{E}-01$ \\
281 & 65 & 153 & 16 & $0.2565903 \mathrm{E}-07$ & $0.5553079 \mathrm{E}-01$ \\
537 & 65 & 281 & 16 & $0.2142430 \mathrm{E}-07$ & $0.9948325 \mathrm{E}-01$ \\
1049 & 65 & 537 & 16 & $0.2793553 \mathrm{E}-07$ & $0.1880572 \mathrm{E}+00$ \\
2073 & 65 & 1049 & 14 & $0.3109474 \mathrm{E}-07$ & $0.3246143 \mathrm{E}+00$ \\
4121 & 65 & 2073 & 15 & $0.3043157 \mathrm{E}-07$ & $0.7092645 \mathrm{E}+00$ \\
153 & 129 & 89 & 16 & $0.8448479 \mathrm{E}-08$ & $0.6391597 \mathrm{E}-01$ \\
281 & 129 & 153 & 17 & $0.8956454 \mathrm{E}-08$ & $0.1127482 \mathrm{E}+00$ \\
537 & 129 & 281 & 17 & $0.9654096 \mathrm{E}-08$ & $0.2068945 \mathrm{E}+00$ \\
1049 & 129 & 537 & 17 & $0.8182551 \mathrm{E}-08$ & $0.4029977 \mathrm{E}+00$ \\
2073 & 129 & 1049 & 17 & $0.1055685 \mathrm{E}-07$ & $0.8235694 \mathrm{E}+00$ \\
4121 & 129 & 2073 & 15 & $0.1141598 \mathrm{E}-07$ & $0.1568456 \mathrm{E}+01$ \\
153 & 257 & 89 & 16 & $0.3722163 \mathrm{E}-08$ & $0.1232737 \mathrm{E}+00$ \\
281 & 257 & 153 & 18 & $0.5343425 \mathrm{E}-08$ & $0.2387325 \mathrm{E}+00$ \\
537 & 257 & 281 & 19 & $0.6715142 \mathrm{E}-08$ & $0.4757280 \mathrm{E}+00$ \\
1049 & 257 & 537 & 20 & $0.3970437 \mathrm{E}-08$ & $0.1051769 \mathrm{E}+01$ \\
2073 & 257 & 1049 & 19 & $0.7038739 \mathrm{E}-08$ & $0.2125283 \mathrm{E}+01$ \\
4121 & 257 & 2073 & 20 & $0.4351956 \mathrm{E}-08$ & $0.4661099 \mathrm{E}+01$ \\
153 & 513 & 89 & 18 & $0.7654136 \mathrm{E}-09$ & $0.2743895 \mathrm{E}+00$ \\
281 & 513 & 153 & 21 & $0.2616080 \mathrm{E}-08$ & $0.5716468 \mathrm{E}+00$ \\
537 & 513 & 281 & 31 & $0.1001862 \mathrm{E}-06$ & $0.1654959 \mathrm{E}+01$ \\
1049 & 513 & 537 & 45 & $0.2129826 \mathrm{E}-06$ & $0.5157828 \mathrm{E}+01$ \\
2073 & 513 & 1049 & 50 & $0.2451359 \mathrm{E}-06$ & $0.1168640 \mathrm{E}+02$ \\
4121 & 513 & 2073 & 50 & $0.2152588 \mathrm{E}-06$ & $0.2433490 \mathrm{E}+02$ \\
153 & 1025 & 89 & 19 & $0.2556742 \mathrm{E}-09$ & $0.6080922 \mathrm{E}+00$ \\
281 & 1025 & 153 & 23 & $0.1654662 \mathrm{E}-08$ & $0.1378273 \mathrm{E}+01$ \\
537 & 1025 & 281 & 50 & $0.6272517 \mathrm{E}-07$ & $0.5847462 \mathrm{E}+01$ \\
1049 & 1025 & 537 & 111 & $0.1797939 \mathrm{E}-06$ & $0.2655321 \mathrm{E}+02$ \\
2073 & 1025 & 1049 & 172 & $0.2869031 \mathrm{E}-06$ & $0.8301551 \mathrm{E}+02$ \\
4121 & 1025 & 2073 & 193 & $0.3516878 \mathrm{E}-06$ & $0.1891160 \mathrm{E}+03$ \\
\hline \hline
\end{tabular}


Tabela A.7: Método multigrid FAS - Caso I - 4p.

\begin{tabular}{cccccc}
\hline \hline imax & jmax & Nx & ciclos V & erro & tempo \\
\hline 153 & 33 & 57 & 14 & $0.6020318 \mathrm{E}-07$ & $0.1759326 \mathrm{E}-01$ \\
281 & 33 & 89 & 14 & $0.5377710 \mathrm{E}-07$ & $0.1741397 \mathrm{E}-01$ \\
537 & 33 & 153 & 14 & $0.5943889 \mathrm{E}-07$ & $0.3124845 \mathrm{E}-01$ \\
1049 & 33 & 281 & 14 & $0.5944006 \mathrm{E}-07$ & $0.4641598 \mathrm{E}-01$ \\
2073 & 33 & 537 & 14 & $0.4855497 \mathrm{E}-07$ & $0.8541733 \mathrm{E}-01$ \\
4121 & 33 & 1049 & 14 & $0.5897942 \mathrm{E}-07$ & $0.1638421 \mathrm{E}+00$ \\
153 & 65 & 57 & 16 & $0.3616609 \mathrm{E}-07$ & $0.2406621 \mathrm{E}-01$ \\
281 & 65 & 89 & 16 & $0.2702686 \mathrm{E}-07$ & $0.3523070 \mathrm{E}-01$ \\
537 & 65 & 153 & 16 & $0.2566294 \mathrm{E}-07$ & $0.5717325 \mathrm{E}-01$ \\
1049 & 65 & 281 & 16 & $0.2793538 \mathrm{E}-07$ & $0.1018941 \mathrm{E}+00$ \\
2073 & 65 & 537 & 16 & $0.2793553 \mathrm{E}-07$ & $0.1909295 \mathrm{E}+00$ \\
4121 & 65 & 1049 & 16 & $0.2174680 \mathrm{E}-07$ & $0.3747404 \mathrm{E}+00$ \\
153 & 129 & 57 & 17 & $0.8741831 \mathrm{E}-08$ & $0.4559082 \mathrm{E}-01$ \\
281 & 129 & 89 & 17 & $0.1209165 \mathrm{E}-07$ & $0.7383955 \mathrm{E}-01$ \\
537 & 129 & 153 & 17 & $0.1010541 \mathrm{E}-07$ & $0.1162127 \mathrm{E}+00$ \\
1049 & 129 & 281 & 17 & $0.9657944 \mathrm{E}-08$ & $0.2097692 \mathrm{E}+00$ \\
2073 & 129 & 537 & 17 & $0.1055677 \mathrm{E}-07$ & $0.4085647 \mathrm{E}+00$ \\
4121 & 129 & 1049 & 17 & $0.1055685 \mathrm{E}-07$ & $0.8930785 \mathrm{E}+00$ \\
153 & 257 & 57 & 17 & $0.4482169 \mathrm{E}-08$ & $0.8625025 \mathrm{E}-01$ \\
281 & 257 & 89 & 19 & $0.4692863 \mathrm{E}-08$ & $0.1483808 \mathrm{E}+00$ \\
537 & 257 & 153 & 20 & $0.5498757 \mathrm{E}-08$ & $0.2654863 \mathrm{E}+00$ \\
1049 & 257 & 281 & 20 & $0.4679465 \mathrm{E}-08$ & $0.5127483 \mathrm{E}+00$ \\
2073 & 257 & 537 & 20 & $0.3715046 \mathrm{E}-08$ & $0.1115219 \mathrm{E}+01$ \\
4121 & 257 & 1049 & 20 & $0.4344473 \mathrm{E}-08$ & $0.2407695 \mathrm{E}+01$ \\
153 & 513 & 57 & 18 & $0.1768197 \mathrm{E}-08$ & $0.1784703 \mathrm{E}+00$ \\
281 & 513 & 89 & 22 & $0.3558656 \mathrm{E}-08$ & $0.3350775 \mathrm{E}+00$ \\
537 & 513 & 153 & 32 & $0.1150562 \mathrm{E}-06$ & $0.8912908 \mathrm{E}+00$ \\
1049 & 513 & 281 & 46 & $0.2091633 \mathrm{E}-06$ & $0.2636433 \mathrm{E}+01$ \\
2073 & 513 & 537 & 51 & $0.2126426 \mathrm{E}-06$ & $0.6207307 \mathrm{E}+01$ \\
4121 & 513 & 1049 & 49 & $0.2777622 \mathrm{E}-06$ & $0.1234071 \mathrm{E}+02$ \\
153 & 1025 & 57 & 20 & $0.3219387 \mathrm{E}-09$ & $0.4005781 \mathrm{E}+00$ \\
281 & 1025 & 89 & 24 & $0.2820927 \mathrm{E}-08$ & $0.8128633 \mathrm{E}+00$ \\
537 & 1025 & 153 & 52 & $0.6432398 \mathrm{E}-07$ & $0.3283207 \mathrm{E}+01$ \\
1049 & 1025 & 281 & 113 & $0.1863263 \mathrm{E}-06$ & $0.1429401 \mathrm{E}+02$ \\
2073 & 1025 & 537 & 173 & $0.3027906 \mathrm{E}-06$ & $0.4481797 \mathrm{E}+02$ \\
4121 & 1025 & 1049 & 194 & $0.3473279 \mathrm{E}-06$ & $0.1013286 \mathrm{E}+03$ \\
\hline \hline
\end{tabular}


Tabela A.8: Método multigrid FAS - Caso I - 8p.

\begin{tabular}{cccccc}
\hline \hline imax & jmax & Nx & ciclos V & erro & tempo \\
\hline 281 & 33 & 57 & 14 & $0.6284475 \mathrm{E}-07$ & $0.3586322 \mathrm{E}-01$ \\
537 & 33 & 89 & 14 & $0.6020956 \mathrm{E}-07$ & $0.3754425 \mathrm{E}-01$ \\
1049 & 33 & 153 & 14 & $0.5943889 \mathrm{E}-07$ & $0.3291494 \mathrm{E}-01$ \\
2073 & 33 & 281 & 14 & $0.5944006 \mathrm{E}-07$ & $0.5596244 \mathrm{E}-01$ \\
4121 & 33 & 537 & 14 & $0.6052936 \mathrm{E}-07$ & $0.9456450 \mathrm{E}-01$ \\
281 & 65 & 57 & 16 & $0.3625411 \mathrm{E}-07$ & $0.4317033 \mathrm{E}-01$ \\
537 & 65 & 89 & 16 & $0.2825189 \mathrm{E}-07$ & $0.5560231 \mathrm{E}-01$ \\
1049 & 65 & 153 & 16 & $0.2794953 \mathrm{E}-07$ & $0.6760442 \mathrm{E}-01$ \\
2073 & 65 & 281 & 16 & $0.2793538 \mathrm{E}-07$ & $0.1098903 \mathrm{E}+00$ \\
4121 & 65 & 537 & 16 & $0.2793553 \mathrm{E}-07$ & $0.2010750 \mathrm{E}+00$ \\
281 & 129 & 57 & 17 & $0.1937734 \mathrm{E}-07$ & $0.5490094 \mathrm{E}-01$ \\
537 & 129 & 89 & 17 & $0.1338213 \mathrm{E}-07$ & $0.7193148 \mathrm{E}-01$ \\
1049 & 129 & 153 & 17 & $0.1062570 \mathrm{E}-07$ & $0.1211697 \mathrm{E}+00$ \\
2073 & 129 & 281 & 17 & $0.1059075 \mathrm{E}-07$ & $0.2448082 \mathrm{E}+00$ \\
4121 & 129 & 537 & 17 & $0.1055677 \mathrm{E}-07$ & $0.5000733 \mathrm{E}+00$ \\
281 & 257 & 57 & 19 & $0.1195974 \mathrm{E}-07$ & $0.1070983 \mathrm{E}+00$ \\
537 & 257 & 89 & 20 & $0.1210333 \mathrm{E}-07$ & $0.1659457 \mathrm{E}+00$ \\
1049 & 257 & 153 & 20 & $0.8637744 \mathrm{E}-08$ & $0.3008227 \mathrm{E}+00$ \\
2073 & 257 & 281 & 20 & $0.4811730 \mathrm{E}-08$ & $0.6244125 \mathrm{E}+00$ \\
4121 & 257 & 537 & 20 & $0.4515873 \mathrm{E}-08$ & $0.1403498 \mathrm{E}+01$ \\
281 & 513 & 57 & 23 & $0.9504480 \mathrm{E}-08$ & $0.2431736 \mathrm{E}+00$ \\
537 & 513 & 89 & 34 & $0.1432263 \mathrm{E}-06$ & $0.6056430 \mathrm{E}+00$ \\
1049 & 513 & 153 & 48 & $0.2044892 \mathrm{E}-06$ & $0.1624251 \mathrm{E}+01$ \\
2073 & 513 & 281 & 52 & $0.2181490 \mathrm{E}-06$ & $0.3658203 \mathrm{E}+01$ \\
4121 & 513 & 537 & 50 & $0.2474325 \mathrm{E}-06$ & $0.7308401 \mathrm{E}+01$ \\
281 & 1025 & 57 & 26 & $0.5868727 \mathrm{E}-08$ & $0.6483875 \mathrm{E}+00$ \\
537 & 1025 & 89 & 56 & $0.6595084 \mathrm{E}-07$ & $0.2356579 \mathrm{E}+01$ \\
1049 & 1025 & 153 & 117 & $0.1991059 \mathrm{E}-06$ & $0.9225366 \mathrm{E}+01$ \\
2073 & 1025 & 281 & 177 & $0.2939245 \mathrm{E}-06$ & $0.2759287 \mathrm{E}+02$ \\
4121 & 1025 & 537 & 196 & $0.3475327 \mathrm{E}-06$ & $0.6140729 \mathrm{E}+02$ \\
\hline \hline & & & & &
\end{tabular}


Tabela A.9: Método multigrid CS - Caso II - 1p.

\begin{tabular}{cccccc}
\hline \hline imax & jmax & Nx & ciclos V & erro & tempo \\
\hline 153 & 33 & 153 & 58 & $0.7529777 \mathrm{E}-04$ & $0.7501704 \mathrm{E}-01$ \\
281 & 33 & 281 & 57 & $0.7498207 \mathrm{E}-04$ & $0.1362002 \mathrm{E}+00$ \\
537 & 33 & 537 & 57 & $0.7574629 \mathrm{E}-04$ & $0.2612657 \mathrm{E}+00$ \\
1049 & 33 & 1049 & 58 & $0.7549732 \mathrm{E}-04$ & $0.5204268 \mathrm{E}+00$ \\
2073 & 33 & 2073 & 58 & $0.7513563 \mathrm{E}-04$ & $0.1040328 \mathrm{E}+01$ \\
4121 & 33 & 4121 & 53 & $0.7492251 \mathrm{E}-04$ & $0.2033983 \mathrm{E}+01$ \\
153 & 65 & 153 & 65 & $0.8597664 \mathrm{E}-05$ & $0.1648015 \mathrm{E}+00$ \\
281 & 65 & 281 & 66 & $0.9957197 \mathrm{E}-05$ & $0.3094482 \mathrm{E}+00$ \\
537 & 65 & 537 & 66 & $0.9891191 \mathrm{E}-05$ & $0.5937297 \mathrm{E}+00$ \\
1049 & 65 & 1049 & 64 & $0.1001148 \mathrm{E}-04$ & $0.1163610 \mathrm{E}+01$ \\
2073 & 65 & 2073 & 65 & $0.9987804 \mathrm{E}-05$ & $0.2514736 \mathrm{E}+01$ \\
4121 & 65 & 4121 & 66 & $0.9927120 \mathrm{E}-05$ & $0.5729618 \mathrm{E}+01$ \\
153 & 129 & 153 & 74 & $0.4262657 \mathrm{E}-06$ & $0.3690027 \mathrm{E}+00$ \\
281 & 129 & 281 & 73 & $0.1026044 \mathrm{E}-05$ & $0.6736243 \mathrm{E}+00$ \\
537 & 129 & 537 & 74 & $0.1279991 \mathrm{E}-05$ & $0.1397059 \mathrm{E}+01$ \\
1049 & 129 & 1049 & 74 & $0.1270133 \mathrm{E}-05$ & $0.3077175 \mathrm{E}+01$ \\
2073 & 129 & 2073 & 70 & $0.1284306 \mathrm{E}-05$ & $0.6519454 \mathrm{E}+01$ \\
4121 & 129 & 4121 & 73 & $0.1283476 \mathrm{E}-05$ & $0.1459971 \mathrm{E}+02$ \\
153 & 257 & 153 & 79 & $0.1466344 \mathrm{E}-07$ & $0.7949520 \mathrm{E}+00$ \\
281 & 257 & 281 & 82 & $0.4684196 \mathrm{E}-07$ & $0.1605769 \mathrm{E}+01$ \\
537 & 257 & 537 & 82 & $0.1243518 \mathrm{E}-06$ & $0.3567344 \mathrm{E}+01$ \\
1049 & 257 & 1049 & 81 & $0.1622305 \mathrm{E}-06$ & $0.7762618 \mathrm{E}+01$ \\
2073 & 257 & 2073 & 82 & $0.1609091 \mathrm{E}-06$ & $0.1653389 \mathrm{E}+02$ \\
4121 & 257 & 4121 & 77 & $0.1624732 \mathrm{E}-06$ & $0.3341155 \mathrm{E}+02$ \\
153 & 513 & 153 & 83 & $0.4691201 \mathrm{E}-09$ & $0.1763035 \mathrm{E}+01$ \\
281 & 513 & 281 & 86 & $0.1580681 \mathrm{E}-08$ & $0.3896707 \mathrm{E}+01$ \\
537 & 513 & 537 & 89 & $0.5458426 \mathrm{E}-08$ & $0.8719810 \mathrm{E}+01$ \\
1049 & 513 & 1049 & 99 & $0.7387885 \mathrm{E}-06$ & $0.2002103 \mathrm{E}+02$ \\
2073 & 513 & 2073 & 145 & $0.1211354 \mathrm{E}-05$ & $0.6050868 \mathrm{E}+02$ \\
4121 & 513 & 4121 & 146 & $0.1348984 \mathrm{E}-05$ & $0.1306389 \mathrm{E}+03$ \\
153 & 1025 & 153 & 87 & $0.1475159 \mathrm{E}-10$ & $0.4289246 \mathrm{E}+01$ \\
281 & 1025 & 281 & 91 & $0.5032975 \mathrm{E}-10$ & $0.9306723 \mathrm{E}+01$ \\
537 & 1025 & 537 & 94 & $0.1751977 \mathrm{E}-09$ & $0.1951778 \mathrm{E}+02$ \\
1049 & 1025 & 1049 & 161 & $0.4549111 \mathrm{E}-06$ & $0.6753463 \mathrm{E}+02$ \\
2073 & 1025 & 2073 & 364 & $0.1678464 \mathrm{E}-05$ & $0.3094769 \mathrm{E}+03$ \\
4121 & 1025 & 4121 & 537 & $0.2708605 \mathrm{E}-05$ & $0.9719006 \mathrm{E}+03$ \\
\hline \hline
\end{tabular}


Tabela A.10: Método multigrid CS - Caso II - 2p.

\begin{tabular}{cccccc}
\hline \hline imax & jmax & Nx & ciclos V & erro & tempo \\
\hline 153 & 33 & 89 & 58 & $0.7529777 \mathrm{E}-04$ & $0.5154252 \mathrm{E}-01$ \\
281 & 33 & 153 & 57 & $0.7498207 \mathrm{E}-04$ & $0.8518392 \mathrm{E}-01$ \\
537 & 33 & 281 & 57 & $0.7574629 \mathrm{E}-04$ & $0.1471535 \mathrm{E}+00$ \\
1049 & 33 & 537 & 58 & $0.7549732 \mathrm{E}-04$ & $0.2786148 \mathrm{E}+00$ \\
2073 & 33 & 1049 & 58 & $0.7513563 \mathrm{E}-04$ & $0.5329495 \mathrm{E}+00$ \\
4121 & 33 & 2073 & 53 & $0.7492251 \mathrm{E}-04$ & $0.9689997 \mathrm{E}+00$ \\
153 & 65 & 89 & 65 & $0.8597664 \mathrm{E}-05$ & $0.1101591 \mathrm{E}+00$ \\
281 & 65 & 153 & 66 & $0.9957197 \mathrm{E}-05$ & $0.1795127 \mathrm{E}+00$ \\
537 & 65 & 281 & 66 & $0.9891191 \mathrm{E}-05$ & $0.3250997 \mathrm{E}+00$ \\
1049 & 65 & 537 & 64 & $0.1001148 \mathrm{E}-04$ & $0.5908292 \mathrm{E}+00$ \\
2073 & 65 & 1049 & 65 & $0.9987804 \mathrm{E}-05$ & $0.1202185 \mathrm{E}+01$ \\
4121 & 65 & 2073 & 66 & $0.9927120 \mathrm{E}-05$ & $0.2578474 \mathrm{E}+01$ \\
153 & 129 & 89 & 74 & $0.4262657 \mathrm{E}-06$ & $0.2256967 \mathrm{E}+00$ \\
281 & 129 & 153 & 73 & $0.1026044 \mathrm{E}-05$ & $0.3773988 \mathrm{E}+00$ \\
537 & 129 & 281 & 74 & $0.1279991 \mathrm{E}-05$ & $0.7011282 \mathrm{E}+00$ \\
1049 & 129 & 537 & 74 & $0.1270133 \mathrm{E}-05$ & $0.1416752 \mathrm{E}+01$ \\
2073 & 129 & 1049 & 70 & $0.1284306 \mathrm{E}-05$ & $0.2950137 \mathrm{E}+01$ \\
4121 & 129 & 2073 & 73 & $0.1283476 \mathrm{E}-05$ & $0.6855227 \mathrm{E}+01$ \\
153 & 257 & 89 & 79 & $0.1466343 \mathrm{E}-07$ & $0.4728333 \mathrm{E}+00$ \\
281 & 257 & 153 & 82 & $0.4684196 \mathrm{E}-07$ & $0.8431500 \mathrm{E}+00$ \\
537 & 257 & 281 & 82 & $0.1243518 \mathrm{E}-06$ & $0.1647754 \mathrm{E}+01$ \\
1049 & 257 & 537 & 81 & $0.1622292 \mathrm{E}-06$ & $0.3566688 \mathrm{E}+01$ \\
2073 & 257 & 1049 & 82 & $0.1609048 \mathrm{E}-06$ & $0.7913590 \mathrm{E}+01$ \\
4121 & 257 & 2073 & 77 & $0.1624711 \mathrm{E}-06$ & $0.1564270 \mathrm{E}+02$ \\
153 & 513 & 89 & 83 & $0.4691135 \mathrm{E}-09$ & $0.9694926 \mathrm{E}+00$ \\
281 & 513 & 153 & 86 & $0.1580676 \mathrm{E}-08$ & $0.1863276 \mathrm{E}+01$ \\
537 & 513 & 281 & 89 & $0.5457328 \mathrm{E}-08$ & $0.4073543 \mathrm{E}+01$ \\
1049 & 513 & 537 & 107 & $0.3575429 \mathrm{E}-06$ & $0.1073210 \mathrm{E}+02$ \\
2073 & 513 & 1049 & 150 & $0.9331505 \mathrm{E}-06$ & $0.3118576 \mathrm{E}+02$ \\
4121 & 513 & 2073 & 147 & $0.1350466 \mathrm{E}-05$ & $0.6162300 \mathrm{E}+02$ \\
153 & 1025 & 89 & 87 & $0.1475086 \mathrm{E}-10$ & $0.2215356 \mathrm{E}+01$ \\
281 & 1025 & 153 & 91 & $0.5032924 \mathrm{E}-10$ & $0.4514211 \mathrm{E}+01$ \\
537 & 1025 & 281 & 94 & $0.1676548 \mathrm{E}-09$ & $0.9898603 \mathrm{E}+01$ \\
1049 & 1025 & 537 & 174 & $0.2230164 \mathrm{E}-06$ & $0.3640360 \mathrm{E}+02$ \\
2073 & 1025 & 1049 & 399 & $0.6697801 \mathrm{E}-06$ & $0.1676255 \mathrm{E}+03$ \\
4121 & 1025 & 2073 & 594 & $0.1000628 \mathrm{E}-05$ & $0.5078244 \mathrm{E}+03$ \\
\hline \hline
\end{tabular}


Tabela A.11: Método multigrid CS - Caso II - 4p.

\begin{tabular}{cccccc}
\hline \hline imax & jmax & Nx & ciclos V & erro & tempo \\
\hline 153 & 33 & 57 & 58 & $0.7529777 \mathrm{E}-04$ & $0.4486877 \mathrm{E}-01$ \\
281 & 33 & 89 & 57 & $0.7498207 \mathrm{E}-04$ & $0.5814976 \mathrm{E}-01$ \\
537 & 33 & 153 & 57 & $0.7574629 \mathrm{E}-04$ & $0.8922768 \mathrm{E}-01$ \\
1049 & 33 & 281 & 58 & $0.7549732 \mathrm{E}-04$ & $0.1547580 \mathrm{E}+00$ \\
2073 & 33 & 537 & 58 & $0.7513563 \mathrm{E}-04$ & $0.2831892 \mathrm{E}+00$ \\
4121 & 33 & 1049 & 53 & $0.7492251 \mathrm{E}-04$ & $0.4930490 \mathrm{E}+00$ \\
153 & 65 & 57 & 65 & $0.8597664 \mathrm{E}-05$ & $0.7990342 \mathrm{E}-01$ \\
281 & 65 & 89 & 66 & $0.9957197 \mathrm{E}-05$ & $0.1166744 \mathrm{E}+00$ \\
537 & 65 & 153 & 66 & $0.9891191 \mathrm{E}-05$ & $0.1860256 \mathrm{E}+00$ \\
1049 & 65 & 281 & 64 & $0.1001148 \mathrm{E}-04$ & $0.3206238 \mathrm{E}+00$ \\
2073 & 65 & 537 & 65 & $0.9987804 \mathrm{E}-05$ & $0.6071905 \mathrm{E}+00$ \\
4121 & 65 & 1049 & 66 & $0.9927120 \mathrm{E}-05$ & $0.1250806 \mathrm{E}+01$ \\
153 & 129 & 57 & 74 & $0.4262657 \mathrm{E}-06$ & $0.1553675 \mathrm{E}+00$ \\
281 & 129 & 89 & 73 & $0.1026044 \mathrm{E}-05$ & $0.2302034 \mathrm{E}+00$ \\
537 & 129 & 153 & 74 & $0.1279992 \mathrm{E}-05$ & $0.3889893 \mathrm{E}+00$ \\
1049 & 129 & 281 & 74 & $0.1270133 \mathrm{E}-05$ & $0.7090783 \mathrm{E}+00$ \\
2073 & 129 & 537 & 70 & $0.1284306 \mathrm{E}-05$ & $0.1388567 \mathrm{E}+01$ \\
4121 & 129 & 1049 & 73 & $0.1283476 \mathrm{E}-05$ & $0.3441524 \mathrm{E}+01$ \\
153 & 257 & 57 & 79 & $0.1466341 \mathrm{E}-07$ & $0.3053905 \mathrm{E}+00$ \\
281 & 257 & 89 & 82 & $0.4684192 \mathrm{E}-07$ & $0.4924383 \mathrm{E}+00$ \\
537 & 257 & 153 & 82 & $0.1243518 \mathrm{E}-06$ & $0.8497770 \mathrm{E}+00$ \\
1049 & 257 & 281 & 81 & $0.1622273 \mathrm{E}-06$ & $0.1678181 \mathrm{E}+01$ \\
2073 & 257 & 537 & 82 & $0.1609039 \mathrm{E}-06$ & $0.4019672 \mathrm{E}+01$ \\
4121 & 257 & 1049 & 77 & $0.1624735 \mathrm{E}-06$ & $0.8138741 \mathrm{E}+01$ \\
153 & 513 & 57 & 83 & $0.4691019 \mathrm{E}-09$ & $0.6240687 \mathrm{E}+00$ \\
281 & 513 & 89 & 86 & $0.1580666 \mathrm{E}-08$ & $0.1022567 \mathrm{E}+01$ \\
537 & 513 & 153 & 89 & $0.5454864 \mathrm{E}-08$ & $0.2049642 \mathrm{E}+01$ \\
1049 & 513 & 281 & 108 & $0.3948722 \mathrm{E}-06$ & $0.5491249 \mathrm{E}+01$ \\
2073 & 513 & 537 & 151 & $0.9479133 \mathrm{E}-06$ & $0.1627158 \mathrm{E}+02$ \\
4121 & 513 & 1049 & 149 & $0.1203745 \mathrm{E}-05$ & $0.3354005 \mathrm{E}+02$ \\
153 & 1025 & 57 & 87 & $0.1474963 \mathrm{E}-10$ & $0.1358661 \mathrm{E}+01$ \\
281 & 1025 & 89 & 91 & $0.5032822 \mathrm{E}-10$ & $0.2555094 \mathrm{E}+01$ \\
537 & 1025 & 153 & 94 & $0.1525777 \mathrm{E}-09$ & $0.5226666 \mathrm{E}+01$ \\
1049 & 1025 & 281 & 177 & $0.2238918 \mathrm{E}-06$ & $0.2004949 \mathrm{E}+02$ \\
2073 & 1025 & 537 & 402 & $0.6790337 \mathrm{E}-06$ & $0.9285129 \mathrm{E}+02$ \\
4121 & 1025 & 1049 & 596 & $0.1008093 \mathrm{E}-05$ & $0.2789931 \mathrm{E}+03$ \\
\hline \hline
\end{tabular}


Tabela A.12: Método multigrid CS - Caso II - 8p.

\begin{tabular}{cccccc}
\hline \hline imax & jmax & Nx & ciclos V & erro & tempo \\
\hline 281 & 33 & 57 & 57 & $0.7498207 \mathrm{E}-04$ & $0.6328624 \mathrm{E}-01$ \\
537 & 33 & 89 & 57 & $0.7574629 \mathrm{E}-04$ & $0.8266801 \mathrm{E}-01$ \\
1049 & 33 & 153 & 58 & $0.7549732 \mathrm{E}-04$ & $0.1179730 \mathrm{E}+00$ \\
2073 & 33 & 281 & 58 & $0.7513563 \mathrm{E}-04$ & $0.1832178 \mathrm{E}+00$ \\
4121 & 33 & 537 & 53 & $0.7492251 \mathrm{E}-04$ & $0.2636855 \mathrm{E}+00$ \\
281 & 65 & 57 & 66 & $0.9957197 \mathrm{E}-05$ & $0.9186524 \mathrm{E}-01$ \\
537 & 65 & 89 & 66 & $0.9891191 \mathrm{E}-05$ & $0.1342409 \mathrm{E}+00$ \\
1049 & 65 & 153 & 64 & $0.1001148 \mathrm{E}-04$ & $0.1931472 \mathrm{E}+00$ \\
2073 & 65 & 281 & 65 & $0.9987804 \mathrm{E}-05$ & $0.3388150 \mathrm{E}+00$ \\
4121 & 65 & 537 & 66 & $0.9927120 \mathrm{E}-05$ & $0.6657170 \mathrm{E}+00$ \\
281 & 129 & 57 & 73 & $0.1026044 \mathrm{E}-05$ & $0.1710752 \mathrm{E}+00$ \\
537 & 129 & 89 & 74 & $0.1279991 \mathrm{E}-05$ & $0.2525536 \mathrm{E}+00$ \\
1049 & 129 & 153 & 74 & $0.1270133 \mathrm{E}-05$ & $0.4038437 \mathrm{E}+00$ \\
2073 & 129 & 281 & 70 & $0.1284306 \mathrm{E}-05$ & $0.7518942 \mathrm{E}+00$ \\
4121 & 129 & 537 & 73 & $0.1283476 \mathrm{E}-05$ & $0.1953233 \mathrm{E}+01$ \\
281 & 257 & 57 & 82 & $0.4684175 \mathrm{E}-07$ & $0.3364425 \mathrm{E}+00$ \\
537 & 257 & 89 & 82 & $0.1243518 \mathrm{E}-06$ & $0.5254183 \mathrm{E}+00$ \\
1049 & 257 & 153 & 81 & $0.1622200 \mathrm{E}-06$ & $0.9904906 \mathrm{E}+00$ \\
2073 & 257 & 281 & 82 & $0.1608971 \mathrm{E}-06$ & $0.2307756 \mathrm{E}+01$ \\
4121 & 257 & 537 & 77 & $0.1624659 \mathrm{E}-06$ & $0.4778107 \mathrm{E}+01$ \\
281 & 513 & 57 & 86 & $0.1580652 \mathrm{E}-08$ & $0.7116330 \mathrm{E}+00$ \\
537 & 513 & 89 & 89 & $0.5440580 \mathrm{E}-08$ & $0.1342526 \mathrm{E}+01$ \\
1049 & 513 & 153 & 111 & $0.4269189 \mathrm{E}-06$ & $0.3436228 \mathrm{E}+01$ \\
2073 & 513 & 281 & 153 & $0.9904764 \mathrm{E}-06$ & $0.9922065 \mathrm{E}+01$ \\
4121 & 513 & 537 & 152 & $0.1075750 \mathrm{E}-05$ & $0.2070307 \mathrm{E}+02$ \\
281 & 1025 & 57 & 91 & $0.5032581 \mathrm{E}-10$ & $0.1999476 \mathrm{E}+01$ \\
537 & 1025 & 89 & 94 & $0.9135179 \mathrm{E}-10$ & $0.3633752 \mathrm{E}+01$ \\
1049 & 1025 & 153 & 182 & $0.2405467 \mathrm{E}-06$ & $0.1343889 \mathrm{E}+02$ \\
2073 & 1025 & 281 & 409 & $0.6790172 \mathrm{E}-06$ & $0.6020926 \mathrm{E}+02$ \\
4121 & 1025 & 537 & 601 & $0.1011428 \mathrm{E}-05$ & $0.1766258 \mathrm{E}+03$ \\
\hline \hline & & & & &
\end{tabular}


Tabela A.13: Método multigrid FAS - Caso II - 1p.

\begin{tabular}{cccccc}
\hline \hline imax & jmax & Nx & ciclos V & erro & tempo \\
\hline 153 & 33 & 153 & 10 & $0.7530031 \mathrm{E}-04$ & $0.1764971 \mathrm{E}-01$ \\
281 & 33 & 281 & 10 & $0.7498368 \mathrm{E}-04$ & $0.3243649 \mathrm{E}-01$ \\
537 & 33 & 537 & 10 & $0.7574968 \mathrm{E}-04$ & $0.6236202 \mathrm{E}-01$ \\
1049 & 33 & 1049 & 10 & $0.7550028 \mathrm{E}-04$ & $0.1219865 \mathrm{E}+00$ \\
2073 & 33 & 2073 & 10 & $0.7513708 \mathrm{E}-04$ & $0.2421178 \mathrm{E}+00$ \\
4121 & 33 & 4121 & 8 & $0.7493047 \mathrm{E}-04$ & $0.4008237 \mathrm{E}+00$ \\
153 & 65 & 153 & 11 & $0.8597172 \mathrm{E}-05$ & $0.3805357 \mathrm{E}-01$ \\
281 & 65 & 281 & 11 & $0.9957666 \mathrm{E}-05$ & $0.6999350 \mathrm{E}-01$ \\
537 & 65 & 537 & 11 & $0.9891440 \mathrm{E}-05$ & $0.1346345 \mathrm{E}+00$ \\
1049 & 65 & 1049 & 11 & $0.1001193 \mathrm{E}-04$ & $0.2651750 \mathrm{E}+00$ \\
2073 & 65 & 2073 & 11 & $0.9988335 \mathrm{E}-05$ & $0.5380882 \mathrm{E}+00$ \\
4121 & 65 & 4121 & 11 & $0.9927556 \mathrm{E}-05$ & $0.1137974 \mathrm{E}+01$ \\
153 & 129 & 153 & 12 & $0.4262996 \mathrm{E}-06$ & $0.7970220 \mathrm{E}-01$ \\
281 & 129 & 281 & 12 & $0.1023893 \mathrm{E}-05$ & $0.1473103 \mathrm{E}+00$ \\
537 & 129 & 537 & 12 & $0.1291480 \mathrm{E}-05$ & $0.2881993 \mathrm{E}+00$ \\
1049 & 129 & 1049 & 12 & $0.1291153 \mathrm{E}-05$ & $0.5938135 \mathrm{E}+00$ \\
2073 & 129 & 2073 & 12 & $0.1276435 \mathrm{E}-05$ & $0.1245459 \mathrm{E}+01$ \\
4121 & 129 & 4121 & 12 & $0.1276435 \mathrm{E}-05$ & $0.2666197 \mathrm{E}+01$ \\
153 & 257 & 153 & 13 & $0.1467087 \mathrm{E}-07$ & $0.1728272 \mathrm{E}+00$ \\
281 & 257 & 281 & 14 & $0.6575607 \mathrm{E}-07$ & $0.3492137 \mathrm{E}+00$ \\
537 & 257 & 537 & 25 & $0.8888277 \mathrm{E}-06$ & $0.1271879 \mathrm{E}+01$ \\
1049 & 257 & 1049 & 34 & $0.1958081 \mathrm{E}-05$ & $0.3689210 \mathrm{E}+01$ \\
2073 & 257 & 2073 & 34 & $0.2120535 \mathrm{E}-05$ & $0.7781671 \mathrm{E}+01$ \\
4121 & 257 & 4121 & 34 & $0.1764646 \mathrm{E}-05$ & $0.1658198 \mathrm{E}+02$ \\
153 & 513 & 153 & 13 & $0.4717405 \mathrm{E}-09$ & $0.3558702 \mathrm{E}+00$ \\
281 & 513 & 281 & 16 & $0.8845562 \mathrm{E}-07$ & $0.8629307 \mathrm{E}+00$ \\
537 & 513 & 537 & 41 & $0.6685250 \mathrm{E}-06$ & $0.4573639 \mathrm{E}+01$ \\
1049 & 513 & 1049 & 91 & $0.1839783 \mathrm{E}-05$ & $0.2097612 \mathrm{E}+02$ \\
2073 & 513 & 2073 & 131 & $0.3171133 \mathrm{E}-05$ & $0.6183392 \mathrm{E}+02$ \\
4121 & 513 & 4121 & 131 & $0.3460591 \mathrm{E}-05$ & $0.1337416 \mathrm{E}+03$ \\
153 & 1025 & 153 & 13 & $0.1530119 \mathrm{E}-10$ & $0.7755570 \mathrm{E}+00$ \\
281 & 1025 & 281 & 17 & $0.3615211 \mathrm{E}-07$ & $0.2001900 \mathrm{E}+01$ \\
537 & 1025 & 537 & 51 & $0.2187208 \mathrm{E}-06$ & $0.1227295 \mathrm{E}+02$ \\
1049 & 1025 & 1049 & 153 & $0.7979892 \mathrm{E}-06$ & $0.7317441 \mathrm{E}+02$ \\
2073 & 1025 & 2073 & 356 & $0.1997563 \mathrm{E}-05$ & $0.3449505 \mathrm{E}+03$ \\
4121 & 1025 & 4121 & 524 & $0.3214162 \mathrm{E}-05$ & $0.1091096 \mathrm{E}+04$ \\
\hline \hline
\end{tabular}


Tabela A.14: Método multigrid FAS - Caso II - 2p.

\begin{tabular}{cccccc}
\hline \hline imax & jmax & Nx & ciclos V & erro & tempo \\
\hline 153 & 33 & 89 & 13 & $0.7530579 \mathrm{E}-04$ & $0.1561904 \mathrm{E}-01$ \\
281 & 33 & 153 & 13 & $0.7491882 \mathrm{E}-04$ & $0.2493352 \mathrm{E}-01$ \\
537 & 33 & 281 & 13 & $0.7574732 \mathrm{E}-04$ & $0.4425079 \mathrm{E}-01$ \\
1049 & 33 & 537 & 12 & $0.7549828 \mathrm{E}-04$ & $0.7693702 \mathrm{E}-01$ \\
2073 & 33 & 1049 & 13 & $0.7513579 \mathrm{E}-04$ & $0.1587480 \mathrm{E}+00$ \\
4121 & 33 & 2073 & 13 & $0.7494426 \mathrm{E}-04$ & $0.3143620 \mathrm{E}+00$ \\
153 & 65 & 89 & 15 & $0.8589688 \mathrm{E}-05$ & $0.3171349 \mathrm{E}-01$ \\
281 & 65 & 153 & 15 & $0.9955449 \mathrm{E}-05$ & $0.5398983 \mathrm{E}-01$ \\
537 & 65 & 281 & 15 & $0.9883574 \mathrm{E}-05$ & $0.9762305 \mathrm{E}-01$ \\
1049 & 65 & 537 & 15 & $0.1001149 \mathrm{E}-04$ & $0.1848205 \mathrm{E}+00$ \\
2073 & 65 & 1049 & 14 & $0.9987861 \mathrm{E}-05$ & $0.3383878 \mathrm{E}+00$ \\
4121 & 65 & 2073 & 15 & $0.9927164 \mathrm{E}-05$ & $0.7457238 \mathrm{E}+00$ \\
153 & 129 & 89 & 16 & $0.4255575 \mathrm{E}-06$ & $0.6481862 \mathrm{E}-01$ \\
281 & 129 & 153 & 17 & $0.1019693 \mathrm{E}-05$ & $0.1151448 \mathrm{E}+00$ \\
537 & 129 & 281 & 17 & $0.1275234 \mathrm{E}-05$ & $0.2106612 \mathrm{E}+00$ \\
1049 & 129 & 537 & 17 & $0.1267345 \mathrm{E}-05$ & $0.4088175 \mathrm{E}+00$ \\
2073 & 129 & 1049 & 18 & $0.1284244 \mathrm{E}-05$ & $0.9010507 \mathrm{E}+00$ \\
4121 & 129 & 2073 & 16 & $0.1283410 \mathrm{E}-05$ & $0.1686770 \mathrm{E}+01$ \\
153 & 257 & 89 & 17 & $0.1386420 \mathrm{E}-07$ & $0.1324152 \mathrm{E}+00$ \\
281 & 257 & 153 & 20 & $0.4156255 \mathrm{E}-07$ & $0.2661148 \mathrm{E}+00$ \\
537 & 257 & 281 & 28 & $0.3409601 \mathrm{E}-06$ & $0.6905995 \mathrm{E}+00$ \\
1049 & 257 & 537 & 39 & $0.5110143 \mathrm{E}-06$ & $0.1994185 \mathrm{E}+01$ \\
2073 & 257 & 1049 & 38 & $0.8166480 \mathrm{E}-06$ & $0.4193427 \mathrm{E}+01$ \\
4121 & 257 & 2073 & 36 & $0.9357303 \mathrm{E}-06$ & $0.8300098 \mathrm{E}+01$ \\
153 & 513 & 89 & 18 & $0.7788062 \mathrm{E}-09$ & $0.2750725 \mathrm{E}+00$ \\
281 & 513 & 153 & 22 & $0.4363890 \mathrm{E}-08$ & $0.5948675 \mathrm{E}+00$ \\
537 & 513 & 281 & 47 & $0.2120069 \mathrm{E}-06$ & $0.2494290 \mathrm{E}+01$ \\
1049 & 513 & 537 & 101 & $0.7296618 \mathrm{E}-06$ & $0.1137390 \mathrm{E}+02$ \\
2073 & 513 & 1049 & 146 & $0.1180528 \mathrm{E}-05$ & $0.3386182 \mathrm{E}+02$ \\
4121 & 513 & 2073 & 143 & $0.1652257 \mathrm{E}-05$ & $0.6802089 \mathrm{E}+02$ \\
153 & 1025 & 89 & 19 & $0.2557170 \mathrm{E}-09$ & $0.6159335 \mathrm{E}+00$ \\
281 & 1025 & 153 & 24 & $0.1132313 \mathrm{E}-08$ & $0.1419636 \mathrm{E}+01$ \\
537 & 1025 & 281 & 58 & $0.7372198 \mathrm{E}-07$ & $0.6905409 \mathrm{E}+01$ \\
1049 & 1025 & 537 & 170 & $0.3030052 \mathrm{E}-06$ & $0.4076409 \mathrm{E}+02$ \\
2073 & 1025 & 1049 & 392 & $0.7835168 \mathrm{E}-06$ & $0.1909921 \mathrm{E}+03$ \\
4121 & 1025 & 2073 & 579 & $0.1235800 \mathrm{E}-05$ & $0.5900468 \mathrm{E}+03$ \\
\hline \hline
\end{tabular}


Tabela A.15: Método multigrid FAS - Caso II - 4p.

\begin{tabular}{cccccc}
\hline \hline imax & jmax & Nx & ciclos V & erro & tempo \\
\hline 153 & 33 & 57 & 13 & $0.7531421 \mathrm{E}-04$ & $0.1699752 \mathrm{E}-01$ \\
281 & 33 & 89 & 13 & $0.7492553 \mathrm{E}-04$ & $0.1720577 \mathrm{E}-01$ \\
537 & 33 & 153 & 13 & $0.7575366 \mathrm{E}-04$ & $0.2867347 \mathrm{E}-01$ \\
1049 & 33 & 281 & 13 & $0.7549816 \mathrm{E}-04$ & $0.4622024 \mathrm{E}-01$ \\
2073 & 33 & 537 & 13 & $0.7513579 \mathrm{E}-04$ & $0.9131771 \mathrm{E}-01$ \\
4121 & 33 & 1049 & 13 & $0.7494426 \mathrm{E}-04$ & $0.1626515 \mathrm{E}+00$ \\
153 & 65 & 57 & 15 & $0.8583256 \mathrm{E}-05$ & $0.2923203 \mathrm{E}-01$ \\
281 & 65 & 89 & 15 & $0.9945886 \mathrm{E}-05$ & $0.3974891 \mathrm{E}-01$ \\
537 & 65 & 153 & 15 & $0.9883966 \mathrm{E}-05$ & $0.5657643 \mathrm{E}-01$ \\
1049 & 65 & 281 & 15 & $0.1001220 \mathrm{E}-04$ & $0.9991378 \mathrm{E}-01$ \\
2073 & 65 & 537 & 15 & $0.9987852 \mathrm{E}-05$ & $0.1885410 \mathrm{E}+00$ \\
4121 & 65 & 1049 & 15 & $0.9927164 \mathrm{E}-05$ & $0.3691292 \mathrm{E}+00$ \\
153 & 129 & 57 & 17 & $0.4253291 \mathrm{E}-06$ & $0.5134821 \mathrm{E}-01$ \\
281 & 129 & 89 & 17 & $0.1012709 \mathrm{E}-05$ & $0.7006252 \mathrm{E}-01$ \\
537 & 129 & 153 & 17 & $0.1267178 \mathrm{E}-05$ & $0.1165368 \mathrm{E}+00$ \\
1049 & 129 & 281 & 17 & $0.1264181 \mathrm{E}-05$ & $0.2128553 \mathrm{E}+00$ \\
2073 & 129 & 537 & 18 & $0.1284745 \mathrm{E}-05$ & $0.4389338 \mathrm{E}+00$ \\
4121 & 129 & 1049 & 18 & $0.1283432 \mathrm{E}-05$ & $0.9604915 \mathrm{E}+00$ \\
153 & 257 & 57 & 17 & $0.1239839 \mathrm{E}-07$ & $0.8599591 \mathrm{E}-01$ \\
281 & 257 & 89 & 20 & $0.3232058 \mathrm{E}-07$ & $0.1557421 \mathrm{E}+00$ \\
537 & 257 & 153 & 29 & $0.3802412 \mathrm{E}-06$ & $0.3831223 \mathrm{E}+00$ \\
1049 & 257 & 281 & 39 & $0.6692388 \mathrm{E}-06$ & $0.9766529 \mathrm{E}+00$ \\
2073 & 257 & 537 & 38 & $0.8424250 \mathrm{E}-06$ & $0.2113526 \mathrm{E}+01$ \\
4121 & 257 & 1049 & 36 & $0.8335685 \mathrm{E}-06$ & $0.4299341 \mathrm{E}+01$ \\
153 & 513 & 57 & 18 & $0.1806609 \mathrm{E}-08$ & $0.1777222 \mathrm{E}+00$ \\
281 & 513 & 89 & 22 & $0.1475375 \mathrm{E}-07$ & $0.3390868 \mathrm{E}+00$ \\
537 & 513 & 153 & 48 & $0.2726454 \mathrm{E}-06$ & $0.1332412 \mathrm{E}+01$ \\
1049 & 513 & 281 & 103 & $0.7384035 \mathrm{E}-06$ & $0.5864237 \mathrm{E}+01$ \\
2073 & 513 & 537 & 148 & $0.1130817 \mathrm{E}-05$ & $0.1804909 \mathrm{E}+02$ \\
4121 & 513 & 1049 & 146 & $0.1378548 \mathrm{E}-05$ & $0.3655889 \mathrm{E}+02$ \\
153 & 1025 & 57 & 20 & $0.3220060 \mathrm{E}-09$ & $0.4060308 \mathrm{E}+00$ \\
281 & 1025 & 89 & 24 & $0.3787216 \mathrm{E}-08$ & $0.8146025 \mathrm{E}+00$ \\
537 & 1025 & 153 & 60 & $0.8069868 \mathrm{E}-07$ & $0.3791579 \mathrm{E}+01$ \\
1049 & 1025 & 281 & 173 & $0.3148827 \mathrm{E}-06$ & $0.2187186 \mathrm{E}+02$ \\
2073 & 1025 & 537 & 396 & $0.7851832 \mathrm{E}-06$ & $0.1029622 \mathrm{E}+03$ \\
4121 & 1025 & 1049 & 582 & $0.1231116 \mathrm{E}-05$ & $0.3063692 \mathrm{E}+03$ \\
\hline \hline
\end{tabular}


Tabela A.16: Método multigrid FAS - Caso II - 8p.

\begin{tabular}{cccccc}
\hline \hline imax & jmax & Nx & ciclos V & erro & tempo \\
\hline 281 & 33 & 57 & 13 & $0.7493632 \mathrm{E}-04$ & $0.2225733 \mathrm{E}-01$ \\
537 & 33 & 89 & 13 & $0.7577288 \mathrm{E}-04$ & $0.3369826 \mathrm{E}-01$ \\
1049 & 33 & 153 & 13 & $0.7550351 \mathrm{E}-04$ & $0.4324377 \mathrm{E}-01$ \\
2073 & 33 & 281 & 13 & $0.7513573 \mathrm{E}-04$ & $0.6018972 \mathrm{E}-01$ \\
4121 & 33 & 537 & 13 & $0.7494426 \mathrm{E}-04$ & $0.9572852 \mathrm{E}-01$ \\
281 & 65 & 57 & 15 & $0.9938132 \mathrm{E}-05$ & $0.3433895 \mathrm{E}-01$ \\
537 & 65 & 89 & 15 & $0.9881836 \mathrm{E}-05$ & $0.5207628 \mathrm{E}-01$ \\
1049 & 65 & 153 & 15 & $0.1000717 \mathrm{E}-04$ & $0.6745005 \mathrm{E}-01$ \\
2073 & 65 & 281 & 15 & $0.9988413 \mathrm{E}-05$ & $0.1106239 \mathrm{E}+00$ \\
4121 & 65 & 537 & 15 & $0.9927157 \mathrm{E}-05$ & $0.2007865 \mathrm{E}+00$ \\
281 & 129 & 57 & 18 & $0.1005354 \mathrm{E}-05$ & $0.6887525 \mathrm{E}-01$ \\
537 & 129 & 89 & 18 & $0.1262386 \mathrm{E}-05$ & $0.8933121 \mathrm{E}-01$ \\
1049 & 129 & 153 & 17 & $0.1258086 \mathrm{E}-05$ & $0.1339985 \mathrm{E}+00$ \\
2073 & 129 & 281 & 18 & $0.1279920 \mathrm{E}-05$ & $0.2442263 \mathrm{E}+00$ \\
4121 & 129 & 537 & 18 & $0.1283667 \mathrm{E}-05$ & $0.5394850 \mathrm{E}+00$ \\
281 & 257 & 57 & 21 & $0.2587350 \mathrm{E}-07$ & $0.1185089 \mathrm{E}+00$ \\
537 & 257 & 89 & 31 & $0.4483075 \mathrm{E}-06$ & $0.2547989 \mathrm{E}+00$ \\
1049 & 257 & 153 & 41 & $0.5979733 \mathrm{E}-06$ & $0.5987895 \mathrm{E}+00$ \\
2073 & 257 & 281 & 39 & $0.8011615 \mathrm{E}-06$ & $0.1213236 \mathrm{E}+01$ \\
4121 & 257 & 537 & 38 & $0.6347462 \mathrm{E}-06$ & $0.2613473 \mathrm{E}+01$ \\
281 & 513 & 57 & 24 & $0.2833322 \mathrm{E}-07$ & $0.2608104 \mathrm{E}+00$ \\
537 & 513 & 89 & 52 & $0.2591671 \mathrm{E}-06$ & $0.9163328 \mathrm{E}+00$ \\
1049 & 513 & 153 & 107 & $0.7559773 \mathrm{E}-06$ & $0.3609713 \mathrm{E}+01$ \\
2073 & 513 & 281 & 150 & $0.1221200 \mathrm{E}-05$ & $0.1046771 \mathrm{E}+02$ \\
4121 & 513 & 537 & 148 & $0.1338243 \mathrm{E}-05$ & $0.2166706 \mathrm{E}+02$ \\
281 & 1025 & 57 & 26 & $0.8140361 \mathrm{E}-08$ & $0.6549498 \mathrm{E}+00$ \\
537 & 1025 & 89 & 64 & $0.9298205 \mathrm{E}-07$ & $0.2711895 \mathrm{E}+01$ \\
1049 & 1025 & 153 & 179 & $0.3374853 \mathrm{E}-06$ & $0.1423478 \mathrm{E}+02$ \\
2073 & 1025 & 281 & 404 & $0.7898160 \mathrm{E}-06$ & $0.6254173 \mathrm{E}+02$ \\
4121 & 1025 & 537 & 587 & $0.1254474 \mathrm{E}-05$ & $0.1849059 \mathrm{E}+03$ \\
\hline \hline & & & & &
\end{tabular}


Tabela A.17: Escoamento de Poiseuille - Caso B - 665 pontos - Estratégia de paralelização I.

\begin{tabular}{|c|c|c|c|c|c|c|}
\hline $\operatorname{imax}$ & jmax & versão & $\mathrm{EP}$ & tempo de execução & speedup & eficiência \\
\hline \multirow{8}{*}{665} & \multirow{8}{*}{49} & \multirow{4}{*}{ CS } & 1 & $0.3481731 \mathrm{E}+03$ & - & - \\
\hline & & & 2 & $0.1817167 \mathrm{E}+03$ & $0.1916021 \mathrm{E}+01$ & $0.9580107 \mathrm{E}+00$ \\
\hline & & & 4 & $0.1032328 \mathrm{E}+03$ & $0.3372700 \mathrm{E}+01$ & $0.8431749 \mathrm{E}+00$ \\
\hline & & & 8 & $0.6441349 \mathrm{E}+02$ & $0.5405282 \mathrm{E}+01$ & $0.6756602 \mathrm{E}+00$ \\
\hline & & \multirow{4}{*}{ FAS } & 1 & $0.2898011 \mathrm{E}+03$ & - & - \\
\hline & & & 2 & $0.1639763 \mathrm{E}+03$ & $0.1767335 \mathrm{E}+01$ & $0.8836674 \mathrm{E}+00$ \\
\hline & & & 4 & $0.9557993 \mathrm{E}+02$ & $0.3032029 \mathrm{E}+01$ & $0.7580072 \mathrm{E}+00$ \\
\hline & & & 8 & $0.6047773 \mathrm{E}+02$ & $0.4791865 \mathrm{E}+01$ & $0.5989831 \mathrm{E}+00$ \\
\hline \multirow{8}{*}{665} & \multirow{8}{*}{65} & \multirow{4}{*}{ CS } & 1 & $0.4646081 \mathrm{E}+03$ & - & - \\
\hline & & & 2 & $0.2397098 \mathrm{E}+03$ & $0.1938210 \mathrm{E}+01$ & $0.9691052 \mathrm{E}+00$ \\
\hline & & & 4 & $0.1348645 \mathrm{E}+03$ & $0.3444999 \mathrm{E}+01$ & $0.8612498 \mathrm{E}+00$ \\
\hline & & & 8 & $0.8415074 \mathrm{E}+02$ & $0.5521141 \mathrm{E}+01$ & $0.6901426 \mathrm{E}+00$ \\
\hline & & \multirow{4}{*}{ FAS } & 1 & $0.3888124 \mathrm{E}+03$ & - & - \\
\hline & & & 2 & $0.2195636 \mathrm{E}+03$ & $0.1770842 \mathrm{E}+01$ & $0.8854210 \mathrm{E}+00$ \\
\hline & & & 4 & $0.1263404 \mathrm{E}+03$ & $0.3077500 \mathrm{E}+01$ & $0.7693749 \mathrm{E}+00$ \\
\hline & & & 8 & $0.8075504 \mathrm{E}+02$ & $0.4814714 \mathrm{E}+01$ & $0.6018392 \mathrm{E}+00$ \\
\hline \multirow{8}{*}{665} & \multirow{8}{*}{81} & \multirow{4}{*}{$\mathrm{CS}$} & 1 & $0.6148823 \mathrm{E}+03$ & - & - \\
\hline & & & 2 & $0.3156647 \mathrm{E}+03$ & $0.1947897 \mathrm{E}+01$ & $0.9739485 \mathrm{E}+00$ \\
\hline & & & 4 & $0.1753298 \mathrm{E}+03$ & $0.3507004 \mathrm{E}+01$ & $0.8767510 \mathrm{E}+00$ \\
\hline & & & 8 & $0.1091346 \mathrm{E}+03$ & $0.5634164 \mathrm{E}+01$ & $0.7042705 \mathrm{E}+00$ \\
\hline & & \multirow{4}{*}{ FAS } & 1 & $0.5084488 \mathrm{E}+03$ & - & - \\
\hline & & & 2 & $0.2882906 \mathrm{E}+03$ & $0.1763667 \mathrm{E}+01$ & $0.8818337 \mathrm{E}+00$ \\
\hline & & & 4 & $0.1643412 \mathrm{E}+03$ & $0.3093860 \mathrm{E}+01$ & $0.7734651 \mathrm{E}+00$ \\
\hline & & & 8 & $0.1042490 \mathrm{E}+03$ & $0.4877253 \mathrm{E}+01$ & $0.6096566 \mathrm{E}+00$ \\
\hline
\end{tabular}


Tabela A.18: Escoamento de Poiseuille - Caso B - 1049 pontos - Estratégia de paralelização I.

\begin{tabular}{|c|c|c|c|c|c|c|}
\hline $\operatorname{imax}$ & jmax & " versão & $\overline{\mathrm{EP}}$ & tempo de execução & $\overline{\text { speedup }}$ & eficiência \\
\hline \multirow{8}{*}{1049} & \multirow{8}{*}{49} & \multirow{4}{*}{$\mathrm{CS}$} & 1 & $0.8888809 \mathrm{E}+03$ & - & - \\
\hline & & & 2 & $0.4469607 \mathrm{E}+03$ & $0.1988723 \mathrm{E}+01$ & $0.9943614 \mathrm{E}+00$ \\
\hline & & & 4 & $0.2405579 \mathrm{E}+03$ & $0.3695081 \mathrm{E}+01$ & $0.9237703 \mathrm{E}+00$ \\
\hline & & & 8 & $0.1426463 \mathrm{E}+03$ & $0.6231363 \mathrm{E}+01$ & $0.7789204 \mathrm{E}+00$ \\
\hline & & \multirow{4}{*}{ FAS } & 1 & $0.7372865 \mathrm{E}+03$ & - & - \\
\hline & & & 2 & $0.3974698 \mathrm{E}+03$ & $0.1854950 \mathrm{E}+01$ & $0.9274748 \mathrm{E}+00$ \\
\hline & & & 4 & $0.2221551 \mathrm{E}+03$ & $0.3318791 \mathrm{E}+01$ & $0.8296978 \mathrm{E}+00$ \\
\hline & & & 8 & $0.1340478 \mathrm{E}+03$ & $0.5500178 \mathrm{E}+01$ & $0.6875222 \mathrm{E}+00$ \\
\hline \multirow{8}{*}{1049} & \multirow{8}{*}{65} & \multirow{4}{*}{ CS } & 1 & $0.1211385 \mathrm{E}+04$ & - & - \\
\hline & & & 2 & $0.6064928 \mathrm{E}+03$ & $0.1997360 \mathrm{E}+01$ & $0.9986801 \mathrm{E}+00$ \\
\hline & & & 4 & $0.3261762 \mathrm{E}+03$ & $0.3713896 \mathrm{E}+01$ & $0.9284741 \mathrm{E}+00$ \\
\hline & & & 8 & $0.1951321 E+03$ & $0.6208025 \mathrm{E}+01$ & $0.7760031 \mathrm{E}+00$ \\
\hline & & \multirow{4}{*}{ FAS } & 1 & $0.9782396 \mathrm{E}+03$ & - & - \\
\hline & & & 2 & $0.5384567 \mathrm{E}+03$ & $0.1816747 \mathrm{E}+01$ & $0.9083735 E+00$ \\
\hline & & & 4 & $0.2973240 \mathrm{E}+03$ & $0.3290146 \mathrm{E}+01$ & $0.8225366 \mathrm{E}+00$ \\
\hline & & & 8 & $0.1813274 \mathrm{E}+03$ & $0.5394880 \mathrm{E}+01$ & $0.6743600 \mathrm{E}+00$ \\
\hline \multirow{8}{*}{1049} & \multirow{8}{*}{81} & \multirow{4}{*}{ CS } & 1 & $0.1633572 \mathrm{E}+04$ & - & - \\
\hline & & & 2 & $0.8196118 \mathrm{E}+03$ & $0.1993105 \mathrm{E}+01$ & $0.9965525 \mathrm{E}+00$ \\
\hline & & & 4 & $0.4303212 \mathrm{E}+03$ & $0.3796170 \mathrm{E}+01$ & $0.9490424 \mathrm{E}+00$ \\
\hline & & & 8 & $0.2612074 \mathrm{E}+03$ & $0.6253929 \mathrm{E}+01$ & $0.7817411 \mathrm{E}+00$ \\
\hline & & \multirow{4}{*}{ FAS } & 1 & $0.1290662 \mathrm{E}+04$ & - & - \\
\hline & & & 2 & $0.7160483 \mathrm{E}+03$ & $0.1802479 E+01$ & $0.9012393 \mathrm{E}+00$ \\
\hline & & & 4 & $0.3947976 \mathrm{E}+03$ & $0.3269173 \mathrm{E}+01$ & $0.8172934 \mathrm{E}+00$ \\
\hline & & & 8 & $0.2449487 \mathrm{E}+03$ & $0.5269110 \mathrm{E}+01$ & $0.6586388 \mathrm{E}+00$ \\
\hline
\end{tabular}


Tabela A.19: Escoamento de Poiseuille - Caso B - 1433 pontos - Estratégia de paralelização I.

\begin{tabular}{|c|c|c|c|c|c|c|}
\hline imax & jmax & versão & $\mathrm{EP}$ & tempo de execução & speedup & eficiência \\
\hline \multirow{8}{*}{1433} & \multirow{8}{*}{49} & \multirow{4}{*}{ CS } & 1 & $0.2007797 \mathrm{E}+04$ & . & - \\
\hline & & & 2 & $0.9918511 \mathrm{E}+03$ & $0.2024293 E+01$ & $0.1012146 \mathrm{E}+01$ \\
\hline & & & 4 & $0.5243043 \mathrm{E}+03$ & $0.3829450 \mathrm{E}+01$ & $0.9573624 \mathrm{E}+00$ \\
\hline & & & 8 & $0.3106268 \mathrm{E}+03$ & $0.6463696 \mathrm{E}+01$ & $0.8079620 \mathrm{E}+00$ \\
\hline & & \multirow{4}{*}{ FAS } & 1 & $0.1647167 \mathrm{E}+04$ & - & - \\
\hline & & & 2 & $0.8625355 \mathrm{E}+03$ & $0.1909680 \mathrm{E}+01$ & $0.9548399 \mathrm{E}+00$ \\
\hline & & & 4 & $0.4772130 \mathrm{E}+03$ & $0.3451638 \mathrm{E}+01$ & $0.8629096 \mathrm{E}+00$ \\
\hline & & & 8 & $0.2854939 \mathrm{E}+03$ & $0.5769533 \mathrm{E}+01$ & $0.7211916 \mathrm{E}+00$ \\
\hline \multirow{8}{*}{1433} & \multirow{8}{*}{65} & \multirow{4}{*}{ CS } & 1 & $0.2858628 \mathrm{E}+04$ & - & - \\
\hline & & & 2 & $0.1378509 \mathrm{E}+04$ & $0.2073711 \mathrm{E}+01$ & $0.1036855 \mathrm{E}+01$ \\
\hline & & & 4 & $0.7270271 \mathrm{E}+03$ & $0.3931942 \mathrm{E}+01$ & $0.9829855 E+00$ \\
\hline & & & 8 & $0.4318108 \mathrm{E}+03$ & $0.6620095 \mathrm{E}+01$ & $0.8275119 \mathrm{E}+00$ \\
\hline & & \multirow{4}{*}{ FAS } & 1 & $0.2210480 \mathrm{E}+04$ & - & - \\
\hline & & & 2 & $0.1186052 \mathrm{E}+04$ & $0.1863730 \mathrm{E}+01$ & $0.9318649 \mathrm{E}+00$ \\
\hline & & & 4 & $0.6526051 \mathrm{E}+03$ & $0.3387163 \mathrm{E}+01$ & $0.8467907 \mathrm{E}+00$ \\
\hline & & & 8 & $0.3942293 \mathrm{E}+03$ & $0.5607092 \mathrm{E}+01$ & $0.7008865 E+00$ \\
\hline \multirow{8}{*}{1433} & \multirow{8}{*}{81} & \multirow{4}{*}{$\mathrm{CS}$} & 1 & $0.3931339 \mathrm{E}+04$ & - & - \\
\hline & & & 2 & $0.1862387 \mathrm{E}+04$ & $0.2110914 \mathrm{E}+01$ & $0.1055457 \mathrm{E}+01$ \\
\hline & & & 4 & $0.9624718 \mathrm{E}+03$ & $0.4084627 \mathrm{E}+01$ & $0.1021157 \mathrm{E}+01$ \\
\hline & & & 8 & $0.5703186 \mathrm{E}+03$ & $0.6893233 \mathrm{E}+01$ & $0.8616541 \mathrm{E}+00$ \\
\hline & & \multirow{4}{*}{ FAS } & 1 & $0.2977533 \mathrm{E}+04$ & - & - \\
\hline & & & 2 & $0.1580257 \mathrm{E}+04$ & $0.1884208 \mathrm{E}+01$ & $0.9421041 \mathrm{E}+00$ \\
\hline & & & 4 & $0.8690474 \mathrm{E}+03$ & $0.3426203 \mathrm{E}+01$ & $0.8565508 \mathrm{E}+00$ \\
\hline & & & 8 & $0.5269094 \mathrm{E}+03$ & $0.5650939 \mathrm{E}+01$ & $0.7063674 \mathrm{E}+00$ \\
\hline
\end{tabular}


Tabela A.20: Escoamento de Poiseuille - Caso B - 665 pontos - Estratégia de paralelização II.

\begin{tabular}{|c|c|c|c|c|c|c|}
\hline$\overline{i m a x}$ & $\overline{\text { jmax }}$ & versão & EP & tempo de execução & speedup & eficiência \\
\hline \multirow{8}{*}{665} & \multirow{8}{*}{49} & \multirow{4}{*}{$\mathrm{CS}$} & 1 & $0.3451679 \mathrm{E}+03$ & - & - \\
\hline & & & 2 & $0.1781014 \mathrm{E}+03$ & $0.1938042 \mathrm{E}+01$ & $0.9690209 \mathrm{E}+00$ \\
\hline & & & 4 & $0.9855107 \mathrm{E}+02$ & $0.3502427 \mathrm{E}+01$ & $0.8756067 \mathrm{E}+00$ \\
\hline & & & 8 & $0.5952445 \mathrm{E}+02$ & $0.5798758 \mathrm{E}+01$ & $0.7248448 \mathrm{E}+00$ \\
\hline & & \multirow{4}{*}{ FAS } & 1 & $0.2855771 \mathrm{E}+03$ & - & - \\
\hline & & & 2 & $0.1594045 \mathrm{E}+03$ & $0.1791525 \mathrm{E}+01$ & $0.8957623 \mathrm{E}+00$ \\
\hline & & & 4 & $0.9065458 \mathrm{E}+02$ & $0.3150167 \mathrm{E}+01$ & $0.7875417 \mathrm{E}+00$ \\
\hline & & & 8 & $0.5551062 \mathrm{E}+02$ & $0.5144549 \mathrm{E}+01$ & $0.6430686 \mathrm{E}+00$ \\
\hline \multirow{8}{*}{665} & \multirow{8}{*}{65} & \multirow{4}{*}{ CS } & 1 & $0.4595071 \mathrm{E}+03$ & - & - \\
\hline & & & 2 & $0.2342813 \mathrm{E}+03$ & $0.1961348 E+01$ & $0.9806740 \mathrm{E}+00$ \\
\hline & & & 4 & $0.1285685 \mathrm{E}+03$ & $0.3574025 \mathrm{E}+01$ & $0.8935063 \mathrm{E}+00$ \\
\hline & & & 8 & $0.7780202 \mathrm{E}+02$ & $0.5906108 \mathrm{E}+01$ & $0.7382635 \mathrm{E}+00$ \\
\hline & & \multirow{4}{*}{ FAS } & 1 & $0.3821811 \mathrm{E}+03$ & - & - \\
\hline & & & 2 & $0.2135893 \mathrm{E}+03$ & $0.1789327 \mathrm{E}+01$ & $0.8946637 \mathrm{E}+00$ \\
\hline & & & 4 & $0.1196987 \mathrm{E}+03$ & $0.3192861 \mathrm{E}+01$ & $0.7982152 \mathrm{E}+00$ \\
\hline & & & 8 & $0.7314624 \mathrm{E}+02$ & $0.5224891 \mathrm{E}+01$ & $0.6531114 \mathrm{E}+00$ \\
\hline \multirow{8}{*}{665} & \multirow{8}{*}{81} & \multirow{4}{*}{ CS } & 1 & $0.6070420 \mathrm{E}+03$ & - & - \\
\hline & & & 2 & $0.3095391 \mathrm{E}+03$ & $0.1961116 \mathrm{e}+01$ & $0.9805578 \mathrm{E}+00$ \\
\hline & & & 4 & $0.1668217 \mathrm{E}+03$ & $0.3638867 \mathrm{e}+01$ & $0.9097168 \mathrm{E}+00$ \\
\hline & & & 8 & $0.1005582 \mathrm{E}+03$ & $0.6036722 \mathrm{e}+01$ & $0.7545903 \mathrm{E}+00$ \\
\hline & & \multirow{4}{*}{ FAS } & 1 & $0.5030284 \mathrm{E}+03$ & - & - \\
\hline & & & 2 & $0.2819084 \mathrm{E}+03$ & $0.1784368 \mathrm{E}+01$ & $0.8921842 \mathrm{E}+00$ \\
\hline & & & 4 & $0.1565967 \mathrm{E}+03$ & $0.3212255 \mathrm{E}+01$ & $0.8030637 \mathrm{E}+00$ \\
\hline & & & 8 & $0.9543672 \mathrm{E}+02$ & $0.5270806 \mathrm{E}+01$ & $0.6588507 \mathrm{E}+00$ \\
\hline
\end{tabular}


Tabela A.21: Escoamento de Poiseuille - Caso B - 1049 pontos - Estratégia de paralelização II.

\begin{tabular}{|c|c|c|c|c|c|c|}
\hline imax & jmax & versão & $\mathrm{EP}$ & tempo de execução & speedup & eficiência \\
\hline \multirow{8}{*}{1049} & \multirow{8}{*}{49} & \multirow{4}{*}{$\mathrm{CS}$} & 1 & $0.8801265 \mathrm{E}+03$ & - & - \\
\hline & & & 2 & $0.4377597 \mathrm{E}+03$ & $0.2010525 \mathrm{E}+01$ & $0.1005262 \mathrm{E}+01$ \\
\hline & & & 4 & $0.2323668 \mathrm{E}+03$ & $0.3787660 \mathrm{E}+01$ & $0.9469150 \mathrm{E}+00$ \\
\hline & & & 8 & $0.1340486 \mathrm{E}+03$ & $0.6565727 \mathrm{E}+01$ & $0.8207159 \mathrm{E}+00$ \\
\hline & & \multirow{4}{*}{ FAS } & 1 & $0.7294025 \mathrm{E}+03$ & - & - \\
\hline & & & 2 & $0.3881805 \mathrm{E}+03$ & $0.1879029 \mathrm{E}+01$ & $0.9395145 \mathrm{E}+00$ \\
\hline & & & 4 & $0.2135913 \mathrm{E}+03$ & $0.3414945 \mathrm{E}+01$ & $0.8537362 \mathrm{E}+00$ \\
\hline & & & 8 & $0.1250727 \mathrm{E}+03$ & $0.5831827 \mathrm{E}+01$ & $0.7289784 \mathrm{E}+00$ \\
\hline \multirow{8}{*}{1049} & \multirow{8}{*}{65} & \multirow{4}{*}{ CS } & 1 & $0.1190781 \mathrm{E}+04$ & - & - \\
\hline & & & 2 & $0.5941986 \mathrm{E}+03$ & $0.2004012 \mathrm{E}+01$ & $0.1002006 \mathrm{E}+01$ \\
\hline & & & 4 & $0.3148266 \mathrm{E}+03$ & $0.3782339 \mathrm{E}+01$ & $0.9455848 \mathrm{E}+00$ \\
\hline & & & 8 & $0.1826542 \mathrm{E}+03$ & $0.6519321 \mathrm{E}+01$ & $0.8149151 \mathrm{E}+00$ \\
\hline & & \multirow{4}{*}{ FAS } & 1 & $0.9646505 \mathrm{E}+03$ & - & - \\
\hline & & & 2 & $0.5265837 \mathrm{E}+03$ & $0.1831903 \mathrm{E}+01$ & $0.9159516 \mathrm{E}+00$ \\
\hline & & & 4 & $0.2851126 \mathrm{E}+03$ & $0.3383401 \mathrm{E}+01$ & $0.8458503 \mathrm{E}+00$ \\
\hline & & & 8 & $0.1689583 \mathrm{E}+03$ & $0.5709401 \mathrm{E}+01$ & $0.7136751 \mathrm{E}+00$ \\
\hline \multirow{8}{*}{1049} & \multirow{8}{*}{81} & \multirow{4}{*}{$\mathrm{CS}$} & 1 & $0.1604220 \mathrm{E}+04$ & - & - \\
\hline & & & 2 & $0.7972916 \mathrm{E}+03$ & $0.2012087 \mathrm{E}+01$ & $0.1005262 \mathrm{E}+01$ \\
\hline & & & 4 & $0.4147990 \mathrm{E}+03$ & $0.3867464 \mathrm{E}+01$ & $0.9469150 \mathrm{E}+00$ \\
\hline & & & 8 & $0.2460311 \mathrm{E}+03$ & $0.6520397 \mathrm{E}+01$ & $0.8207159 \mathrm{E}+00$ \\
\hline & & \multirow{4}{*}{ FAS } & 1 & $0.1277071 \mathrm{E}+04$ & - & - \\
\hline & & & 2 & $0.6971038 \mathrm{E}+03$ & $0.1831967 \mathrm{E}+01$ & $0.9159835 \mathrm{E}+00$ \\
\hline & & & 4 & $0.3791670 \mathrm{E}+03$ & $0.3368097 \mathrm{E}+01$ & $0.8420243 E+00$ \\
\hline & & & 8 & $0.2276172 \mathrm{E}+03$ & $0.5610609 \mathrm{E}+01$ & $0.7013262 \mathrm{E}+00$ \\
\hline
\end{tabular}


Tabela A.22: Escoamento de Poiseuille - Caso B - 1433 pontos - Estratégia de paralelização II.

\begin{tabular}{|c|c|c|c|c|c|c|}
\hline$\overline{\text { imax }}$ & $\overline{\text { jmax }}$ & versão & $\overline{\mathrm{EP}}$ & tempo de execução & speedup & eficiência \\
\hline \multirow{8}{*}{1433} & \multirow{8}{*}{49} & \multirow{4}{*}{ CS } & 1 & $0.1972343 \mathrm{E}+04$ & - & - \\
\hline & & & 2 & $0.9800111 \mathrm{E}+03$ & $0.2012572 \mathrm{E}+01$ & $0.1006286 \mathrm{E}+01$ \\
\hline & & & 4 & $0.4991792 \mathrm{E}+03$ & $0.3951172 \mathrm{E}+01$ & $0.9877930 \mathrm{E}+00$ \\
\hline & & & 8 & $0.2853313 \mathrm{E}+03$ & $0.6912467 \mathrm{E}+01$ & $0.8640584 \mathrm{E}+00$ \\
\hline & & \multirow{4}{*}{ FAS } & 1 & $0.1628741 \mathrm{E}+04$ & - & - \\
\hline & & & 2 & $0.8462546 \mathrm{E}+03$ & $0.1924647 \mathrm{E}+01$ & $0.9623235 \mathrm{E}+00$ \\
\hline & & & 4 & $0.4595072 \mathrm{E}+03$ & $0.3544539 E+01$ & $0.8861349 E+00$ \\
\hline & & & 8 & $0.2674921 \mathrm{E}+03$ & $0.6088933 E+01$ & $0.7611166 \mathrm{E}+00$ \\
\hline \multirow{8}{*}{1433} & \multirow{8}{*}{65} & \multirow{4}{*}{ CS } & 1 & $0.2788222 \mathrm{E}+04$ & - & - \\
\hline & & & 2 & $0.1358330 \mathrm{E}+04$ & $0.2052684 \mathrm{E}+01$ & $0.1026342 \mathrm{E}+01$ \\
\hline & & & 4 & $0.7034899 \mathrm{E}+03$ & $0.3963414 \mathrm{E}+01$ & $0.9908536 \mathrm{E}+00$ \\
\hline & & & 8 & $0.4065118 \mathrm{E}+03$ & $0.6858894 \mathrm{E}+01$ & $0.8573618 \mathrm{E}+00$ \\
\hline & & \multirow{4}{*}{ FAS } & 1 & $0.2182619 \mathrm{E}+04$ & - & - \\
\hline & & & 2 & $0.1175523 \mathrm{E}+04$ & $0.1856722 \mathrm{E}+01$ & $0.9283609 E+00$ \\
\hline & & & 4 & $0.6252244 \mathrm{E}+03$ & $0.3490937 \mathrm{E}+01$ & $0.8727342 \mathrm{E}+00$ \\
\hline & & & 8 & $0.3696280 \mathrm{E}+03$ & $0.5904906 \mathrm{E}+01$ & $0.7381133 \mathrm{E}+00$ \\
\hline \multirow{8}{*}{1433} & \multirow{8}{*}{81} & \multirow{4}{*}{ CS } & 1 & $0.3890878 \mathrm{E}+04$ & - & - \\
\hline & & & 2 & $0.1824564 \mathrm{E}+04$ & $0.2132497 \mathrm{E}+01$ & $0.1066249 \mathrm{E}+01$ \\
\hline & & & 4 & $0.9332828 \mathrm{E}+03$ & $0.4169024 \mathrm{E}+01$ & $0.1042256 \mathrm{E}+01$ \\
\hline & & & 8 & $0.5438854 \mathrm{E}+03$ & $0.7153857 \mathrm{E}+01$ & $0.8942321 \mathrm{E}+00$ \\
\hline & & \multirow{4}{*}{ FAS } & 1 & $0.2940207 \mathrm{E}+04$ & - & - \\
\hline & & & 2 & $0.1553330 \mathrm{E}+04$ & $0.1892841 \mathrm{E}+01$ & $0.9464204 \mathrm{E}+00$ \\
\hline & & & 4 & $0.8425933 \mathrm{E}+03$ & $0.3489473 \mathrm{E}+01$ & $0.8723683 \mathrm{E}+00$ \\
\hline & & & 8 & $0.5005657 \mathrm{E}+03$ & $0.5873768 \mathrm{E}+01$ & $0.7342210 \mathrm{E}+00$ \\
\hline
\end{tabular}

